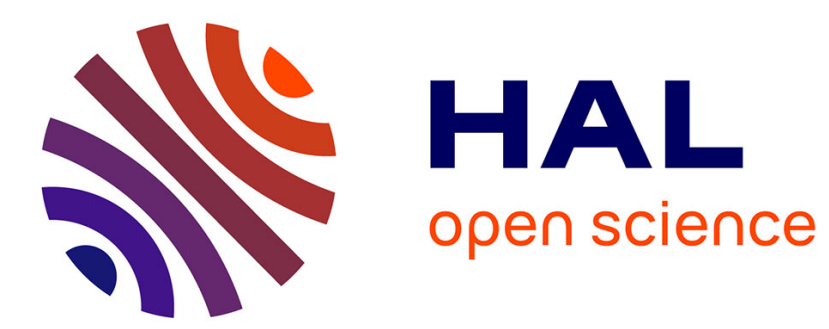

\title{
Large time behaviour for the motion of a solid in a viscous incompressible fluid
}

Sylvain Ervedoza, Debayan Maity, Marius Tucsnak

\section{To cite this version:}

Sylvain Ervedoza, Debayan Maity, Marius Tucsnak. Large time behaviour for the motion of a solid in a viscous incompressible fluid. 2021. hal-02545798v2

\section{HAL Id: hal-02545798 \\ https://hal.science/hal-02545798v2}

Preprint submitted on 12 Feb 2021 (v2), last revised 17 Nov 2022 (v3)

HAL is a multi-disciplinary open access archive for the deposit and dissemination of scientific research documents, whether they are published or not. The documents may come from teaching and research institutions in France or abroad, or from public or private research centers.
L'archive ouverte pluridisciplinaire HAL, est destinée au dépôt et à la diffusion de documents scientifiques de niveau recherche, publiés ou non, émanant des établissements d'enseignement et de recherche français ou étrangers, des laboratoires publics ou privés. 


\title{
Large time behaviour for the motion of a solid in a viscous incompressible fluid*
}

\author{
Sylvain Ervedoza ${ }^{1}$, Debayan Maity ${ }^{2}$, and Marius Tucsnak ${ }^{1}$ \\ ${ }^{1}$ Institut de Mathématiques de Bordeaux UMR 5251, Université de Bordeaux, Bordeaux INP, \\ CNRS, F-33400 Talence, France. \\ ${ }^{2}$ Centre for Applicable Mathematics, TIFR, Post Bag No. 6503, GKVK Post Office, \\ Bangalore-560065, India. \\ sylvain.ervedoza@math.u-bordeaux.fr,debayan@tifrbng.res.in, marius.tucsnak@u-bordeaux.fr
}

February 12, 2021

\begin{abstract}
In this article, we study the long-time behaviour of a system describing the coupled motion of a rigid body and of a viscous incompressible fluid in which the rigid body is contained. We assume that the system formed by the rigid body and the fluid fills the entire space $\mathbb{R}^{3}$. In the case in which the rigid body is a ball, we prove the local existence of mild solutions and, when the initial data are small, the global existence of solutions for this system with a precise description of their large time behavior. Our main result asserts, in particular, that if the initial datum is small enough in suitable norms then the position of the center of the rigid ball converges to some $h_{\infty} \in \mathbb{R}^{3}$ as time goes to infinity. This result contrasts with those known for the analogues of our system in 2 or 1 space dimensions, where it has been proved that the body quits any bounded set, provided that we wait long enough. To achieve this result, we use a "monolithic" type approach, which means that we consider a linearized problem in which the equations of the solid and of the fluid are still coupled. An essential role is played by the properties of the semigroup, called fluid-structure semigroup, associated to this coupled linearized problem. The generator of this semigroup is called the fluidstructure operator. Our main tools are new $L^{p}-L^{q}$ estimates for the fluid-structure semigroup. Note that these estimates are proved for bodies of arbitrary shape. The main ingredients used to study the fluid-structure semigroup and its generator are resolvent estimates which provide both the analyticity of the fluid-structure semigroup (in the spirit of a classical work of Borchers and Sohr) and $L^{p}-L^{q}$ decay estimates (by adapting a strategy due to Iwashita).

Key words. Fluid-structure interaction, Incompressible Navier-Stokes system, Large time behaviour.
\end{abstract}

AMS subject classifications. 35Q35, 35B40, 76D03, 76D05.

${ }^{*}$ The first author has been supported by the CIMI Labex, Toulouse, France, under grant ANR-11-LABX-0040-CIMI. The second author is supported in part by INSPIRE faculty fellowship (IFA18-MA128) and by Department of Atomic Energy, Government of India, under project no. 12-R \& D-TFR-5.01-0520. The second and the third authors are partially supported by the IFCAM project "Analysis, Control and Homogenization of Complex Systems". The third author has been supported by the SingFlows project, grant ANR-18-CE40-0027 of the French National Research Agency (ANR). The first and third authors have been partially supported by the Agence Nationale de la Recherche, Project IFSMACS, grant ANR-15-CE40-0010 and Project TRECOS, grant ANR-20-CE40-0009-01. 


\section{Introduction}

We consider a homogeneous rigid body which occupies at instant $t=0$ a ball $B$ of radius $R>0$ and centered at the origin and we study the motion of this body in a viscous incompressible fluid which fills the remaining part of $\mathbb{R}^{3}$. We denote by $h(t), \mathcal{S}(t), \mathcal{F}(t)$ the position of the centre of the ball, the domain occupied by the solid, which coincides with the ball of radius $R$ centered at $h$, and the domain filled by the fluid, respectively, at instant $t>0$. Moreover, the velocity and pressure fields in the fluid are denoted by $u$ and $p$, respectively. With the above notation, the system describing the motion of the rigid ball in the fluid is

$$
\begin{cases}\partial_{t} u+(u \cdot \nabla) u-\mu \Delta u+\nabla p=0 & (t>0, y \in \mathcal{F}(t)), \\ \operatorname{div} u=0, & (t>0, y \in \mathcal{F}(t)), \\ u(t, y)=\dot{h}(t)+\omega(t) \times(y-h(t)) & (t>0, y \in \partial \mathcal{F}(t)), \\ m \ddot{h}(t)=-\int_{\partial \mathcal{S}(t)} \sigma(u, p) \nu \mathrm{d} s & (t>0), \\ J \dot{\omega}(t)=-\int_{\partial \mathcal{S}(t)}(y-h(t)) \times \sigma(u, p) \nu \mathrm{d} s & (t>0), \\ u(0, y)=u_{0}(y) & (y \in \mathcal{F}(0)), \\ h(0)=0, \dot{h}(0)=\ell_{0}, \omega(0)=\omega_{0} . & \end{cases}
$$

In the above equations, $\omega(t)$ represents the angular velocity of the ball (with respect to its centre) and the fluid is supposed to be homogeneous with density equal to 1 and of constant viscosity $\mu>0$. Moreover, the unit vector field normal to $\partial S(t)$ and directed towards the interior of $\mathcal{S}(t)$ is denoted by $\nu(t, \cdot)$. The constant $m>0$ and the matrix $J$ stand for the mass and the inertia tensor of the rigid body. Since in the above equations the rigid body is a homogeneous ball of radius $R$, we have

$$
J=\frac{2 m R^{2}}{5} \mathbb{I}_{3}
$$

Finally, the Cauchy stress tensor field in the fluid is given by the constitutive law

$$
\sigma(u, p)_{k \ell}=-p \delta_{k \ell}+\mu\left(\frac{\partial u_{k}}{\partial y_{\ell}}+\frac{\partial u_{\ell}}{\partial y_{k}}\right) \quad(1 \leqslant k, \ell \leqslant 3),
$$

where $\delta_{k \ell}$ stands for the Kronecker symbol.

The system (1.1) can be easily transformed into a system in which the fluid equation is written in a fixed spatial domain. Indeed, using the change of frame $x \mapsto y(t, x):=x+h(t)$ and setting

$v_{0}(x)=u_{0}(x), \quad v(t, x)=u(t, x+h(t)), \quad \pi(t, x)=p(t, x+h(t)), \quad \ell(t)=\dot{h}(t) \quad(t>0, x \in \mathcal{F}(0))$, and $E:=\mathcal{F}(0)=\mathbb{R}^{3} \backslash B$, equations (1.1) can be written in the form of the following system of unknowns $v, \pi, \ell$ and $\omega$ :

$$
\begin{cases}\partial_{t} v+[(v-\ell) \cdot \nabla] v-\mu \Delta v+\nabla \pi=0 & (t>0, x \in E), \\ \operatorname{div} v=0, & (t>0, x \in E), \\ v=\ell+\omega \times x & (t>0, x \in \partial E), \\ m \dot{\ell}(t)=-\int_{\partial E} \sigma(v, \pi) \nu \mathrm{d} s, & (t>0), \\ J \dot{\omega}(t)=-\int_{\partial E} x \times \sigma(v, \pi) \nu \mathrm{d} s, & (t>0), \\ v(0, x)=v_{0}(x) & (x \in E), \\ \ell(0)=\ell_{0}, \omega(0)=\omega_{0} . & \end{cases}
$$


As far as we know, the initial and boundary value problem 1.2 has been first studied in Serre 21, where it is proved, in particular, that (1.2) admits global in time weak solutions (of Leray type). The existence and uniqueness of strong solutions, with initial velocity supposed to be small (in the Sobolev space $W^{1,2}$ ) has been first established in Cumsille and Takahashi [4]. For the $L^{p}$ theory for the local in time existence and uniqueness of strong solutions of $(1.2)$, we refer to Geissert, Götze, and Hieber [7. Let us also mention that the analogue of $(1.2)$ when the fluid-rigid body system fills a bounded cavity $\Omega$ (instead of the whole $\mathbb{R}^{3}$ ) has also been studied in a quite important number of papers (see, for instance, Maity and Tucsnak [18 and references therein).

A natural question when considering (1.2) is the large time behaviour of the position of the mass centre of the ball, i.e., of the function $h$ defined by

$$
h(t)=\int_{0}^{t} \ell(s) \mathrm{d} s \quad(t>0) .
$$

It is, in particular, important to establish whether the centre of the rigid ball stabilizes around some position in $\mathbb{R}^{3}$ or its distance to the origin tends to infinity when $t \rightarrow \infty$. As far as we know, this question is open in the three dimensional context of 1.2 . However, if one replaces the rigid ball by an infinite cylinder (so that the fluid can be modeled by the Navier-Stokes equations in two space dimensions) the question is studied in Ervedoza, Hillairet, and Lacave [6, where it is established that the norm of $\ell(t)$ behaves like $\frac{1}{t}$ when $t \rightarrow \infty$, thus not excluding the possibility of an unbounded trajectory of the rigid ball. Other results in the same spirit concern Burgers type models for the fluid, like Vázquez and Zuazua [26, or one dimensional viscous compressible fluids, like Koike [15].

The main novelty brought in by our work is twofold. Firstly, we prove that $(1.2)$ is well-posed (globally in time) for initial data which are small in appropriate $L^{q}$ type spaces. Secondly, by appropriately choosing $q$, we prove that there exists $h_{\infty} \in \mathbb{R}^{3}$ such that $\lim _{t \rightarrow \infty} h(t)=h_{\infty}$, i.e., that the rigid body "stops" as $t \rightarrow \infty$.

To state our main result we first recall that if $G \subset \mathbb{R}^{3}$ is an open set, $q>1$ and $s \in \mathbb{R}$, the notation $L^{q}(G)$ and $W^{s, q}(G)$ stands for the standard Lebesgue and Sobolev-Slobodeckij spaces, respectively. Our main result can be stated as follows:

Theorem 1.1. With the above notation for the set $E$. There exists $\varepsilon_{0}>0$ such that for every $v_{0} \in\left[L^{3}(E)\right]^{3}$ and $\ell_{0}, \omega_{0} \in \mathbb{R}^{3}$ with

$$
\begin{aligned}
& \operatorname{div} v_{0}=0 \text { in } E, \quad v_{0} \cdot \nu=\left(\ell_{0}+\omega_{0} \times x\right) \cdot \nu \text { on } \partial B . \\
& \left\|v_{0}\right\|_{\left[L^{3}(E)\right]^{3}}+\left\|\ell_{0}\right\|_{\mathbb{R}^{3}}+\left\|\omega_{0}\right\|_{\mathbb{R}^{3}} \leqslant \varepsilon_{0},
\end{aligned}
$$

there exists a unique solution $(v, \ell, \omega)$ of $\left[1.2\right.$ in $C^{0}\left([0, \infty) ;\left[L^{3}(E)\right]^{3} \times \mathbb{R}^{3} \times \mathbb{R}^{3}\right)$ such that

$$
\begin{aligned}
\sup _{t>0}\left\{t^{1 / 4}\left(\|v(t)\|_{\left[L^{6}(E)\right]^{3}}+\|\ell(t)\|_{\mathbb{R}^{3}}+\|\omega(t)\|_{\mathbb{R}^{3}}\right)\right. \\
\left.\quad+t^{1 / 2}\left(\|v(t)\|_{\left[L^{\infty}(E)\right]^{3}}+\|\ell(t)\|_{\mathbb{R}^{3}}+\|\omega(t)\|_{\mathbb{R}^{3}}\right)+\min \left\{1, t^{1 / 2}\right\}\|\nabla v(t)\|_{\left[L^{3}(E)\right]^{9}}\right\}<\infty .
\end{aligned}
$$

with

$$
\lim _{t \rightarrow 0}\left(t^{1 / 4}\left(\|v(t)\|_{\left[L^{6}(E)\right]^{3}}+\|\ell(t)\|_{\mathbb{R}^{3}}+\|\omega(t)\|_{\mathbb{R}^{3}}\right)+t^{1 / 2}\|v(t)\|_{\left[L^{\infty}(E)\right]^{3}}+t^{1 / 2}\|\nabla v(t)\|_{\left[L^{3}(E)\right]^{9}}\right)=0 .
$$

Moreover, for $q \in(1,3]$, there exists $\varepsilon_{0}(q) \in\left(0, \varepsilon_{0}\right]$ such that if $v_{0} \in\left[L^{q}(E)\right]^{3} \cap\left[L^{3}(E)\right]^{3}$ satisfies (1.3) and

$$
\left\|v_{0}\right\|_{\left[L^{3}(E)\right]^{3}}+\left\|\ell_{0}\right\|_{\mathbb{R}^{3}}+\left\|\omega_{0}\right\|_{\mathbb{R}^{3}} \leqslant \varepsilon_{0}(q),
$$

then, for every $p \in\left[\max \left\{\frac{3}{2}, q\right\}, \infty\right]$ the solution $(v, \ell, \omega)$ of $(1.2)$ satisfies

$$
\sup _{t>0}\left\{t^{3 / 2(1 / q-1 / p)}\left(\|v(t)\|_{\left[L^{p}(E)\right]^{3}}+\|\ell(t)\|_{\mathbb{R}^{3}}+\|\omega(t)\|_{\mathbb{R}^{3}}\right)\right\}<\infty .
$$


In particular, if $q<3 / 2$, taking $p=\infty$ in 1.6$)$ we have that $\ell \in L^{1}\left([0, \infty) ; \mathbb{R}^{3}\right)$, hence that the position of the centre of the moving rigid ball converges to some point at finite distance $h_{\infty} \in \mathbb{R}^{3}$ as $t \rightarrow \infty$.

As precisely stated in Theorem 8.2 below, the results in Theorem 1.1 can be completed to include a local in time existence result without any smallness assumption on the initial data, see Section 8 for more precise statements.

The proof of Theorem 1.1 is based on decay estimates for the solutions of the linearized version of (1.2). Therefore, an important part of this work is devoted to the study of the semigroup associated to the linearized problem. As shown in the forthcoming sections, this semigroup called the fluidstructure semigroup, and its generator (called the fluid-structure operator) share several important properties of the Stokes semigroup and Stokes operator in an exterior domain. To establish this fact, an essential step consists in proving that the resolvent estimates derived in Iwashita 13 and Giga-Sohr 8 for the Stokes operator also hold for the fluid-structure operator (see also the corresponding estimates for the non-autonomous system describing the Navier-Stokes flow around a rotating obstacle, which have been obtained in Hishida [1] and [12]). Our results on the linearized problem will be derived for a solid of arbitrary shape, opening the way to a generalization of Theorem 1.1 for solids of arbitrary shape. However, the fixed point methodology used in the present paper to pass from the linearized equations to the full nonlinear problem is strongly using the fact that the rigid body is a ball (see the comments in Section 9 below concerning some tracks towards the modification of this procedure for tackling rigid bodies of arbitrary shape).

Note that Theorem 1.1 refers to mild solutions of $\sqrt{1.2}$, i.e., satisfying the integral equation

$$
\left[\begin{array}{c}
v(t, \cdot) \\
\ell(t) \\
\omega(t)
\end{array}\right]=\mathbb{T}_{t}\left[\begin{array}{c}
v_{0} \\
\ell_{0} \\
\omega_{0}
\end{array}\right]+\int_{0}^{t} \mathbb{T}_{t-s} \mathbb{P} f(s) \mathrm{d} s \quad(t \geqslant 0),
$$

where

$$
\left.f(s, x)=-\mathbb{1}_{E}(x)[(v(s, x)-\ell(s)) \cdot \nabla] v(s, x)\right) \quad(x \in E, s \geqslant 0),
$$

$\mathbb{T}=\left(\mathbb{T}_{t}\right)_{t \geqslant 0}$ is the fluid-structure semigroup and $\mathbb{P}$ is a Leray type projector on the space of free divergence vector fields on $\mathbb{R}^{3}$ which coincide with a rigid velocity field on $B$. A precise definition of these objects requires some preparation and notation, so it is postponed to Section 3 . However, we mention here that the roles of the projector $\mathbb{P}$ and of the fluid-structure semigroup in this paper are very close to those played by the Leray projector and the Stokes semigroup in the analysis of the Navier-Stokes equations. Consequently, the construction and study of the fluid-structure semigroup and of its generator are essential steps of our analysis, which are detailed in Sections 4 . 5. 6 and 7 .

The outline of the paper is as follows. In Section 2, we introduce the notation (in particular several function spaces) that will be used throughout the article and we recall several results on the Stokes system exterior domains. In Section 3 we introduce the fluid-structure operator and we give some of its basic properties. Section 4 is devoted to resolvent estimates for the fluid-structure operator. We use existing results on the Stokes system exterior domain to derive our results. In Sections 5 - 6 we show that the fluid-structure operator generates a bounded analytic semigroup on a suitable Banach space. We prove, in particular, $L^{p}-L^{q}$ decay estimates for the fluid-structure semigroup in Section 7. Section 8 is devoted to the proof of Theorem 1.1. In Section 9, we formulate some open problems. Some technical results are collected in Appendix A

\section{Notation and preliminaries}

Throughout this paper, the notation

$$
\mathbb{N}, \mathbb{Z}, \mathbb{R}, \mathbb{C}
$$


stands for the sets of natural numbers (starting with 1), integers, real numbers and complex numbers, respectively. For $n \in \mathbb{N}$, the euclidian norm on $\mathbb{C}^{n}$ will be simply denoted by $|\cdot|$. For $\theta \in(0, \pi)$ we define the sector $\Sigma_{\theta}$ in the complex plane by

$$
\Sigma_{\theta}=\{\lambda \in \mathbb{C} \backslash\{0\} \quad|\quad| \arg \lambda \mid<\theta\} .
$$

Moreover, $\mathbb{Z}_{+}$stands for $\mathbb{N} \cup\{0\}$. For $n, m \in \mathbb{N}, u: \mathbb{R}^{n} \rightarrow \mathbb{R}^{m}$ and $\alpha \in \mathbb{Z}_{+}^{n}$ we set $|\alpha|=\sum_{k=1}^{n} \alpha_{k}$ and we use the notation $\partial^{\alpha} u$ for the partial derivative $\frac{\partial^{|\alpha|} u}{\partial x_{1}^{\alpha_{1}} \ldots x_{n}^{\alpha_{n}}}$.

If $G \subset \mathbb{R}^{3}$ is an open set, $q>1$ and $k \in \mathbb{N}$, we denote the standard Lebesgue and Sobolev spaces by $L^{q}(G)$ and by $W^{k, q}(G)$, respectively. For $s \in \mathbb{R}, W^{s, q}(G)$ denotes the Sobolev-Slobodeckij spaces. The norms on $\left[L^{q}(G)\right]^{n}$ and $\left[W^{k, q}(G)\right]^{n}$ with $n \in \mathbb{N}$, will be denoted by $\|\cdot\|_{q, G}$ and $\|\cdot\|_{k, q, G}$, respectively. When $G=\mathbb{R}^{3}$, these norms will be simply denoted by $\|\cdot\|_{q}$ and $\|\cdot\|_{k, q}$, respectively. Moreover, the space $W_{0}^{k, q}(G)$ is the completion of $C_{0}^{\infty}(G)$ with respect to the $W^{k, q}(G)$ norm.

We use repeatedly below the following well known result due to Bogovskii ([1]):

Lemma 2.1. Let $G$ be a smooth bounded domain in $\mathbb{R}^{3}, q \in(1, \infty)$ and $k \in \mathbb{Z}_{+}$and let

$$
L_{0}^{q}(G)=\left\{f \in L^{q}(G) \mid \int_{G} f \mathrm{~d} x=0\right\} .
$$

Then there exists a linear bounded operator $\mathbb{B}_{G}$ from $\left[W_{0}^{k, q}(G)\right]^{3} \cap\left[L_{0}^{q}(G)\right]^{3}$ to $\left[W_{0}^{k+1, q}(G)\right]^{3}$ such that

$$
\operatorname{div}\left(\mathbb{B}_{G} f\right)=f \quad \text { in } G, \quad\left(f \in\left[W_{0}^{k, q}(G)\right]^{3} \cap\left[L_{0}^{q}(G)\right]^{3}\right) .
$$

We also introduce the homogeneous Sobolev spaces

$$
\widehat{W}^{1, q}(G):=\left\{f \in L_{\mathrm{loc}}^{q}(\bar{G}) \mid \nabla f \in L^{q}(G)^{3}\right\},
$$

with the norm

$$
\|f\|_{\widehat{W}^{1, q}(G)}:=\|\nabla f\|_{q, G},
$$

where we identify elements differing by a constant.

Moreover, the function space

$$
L_{\sigma}^{q}(G)=\overline{\left\{\varphi \in\left[C_{0}^{\infty}(G)\right]^{3} \mid \operatorname{div} \varphi=0\right\}^{\|\cdot\|_{q, G}},}
$$

will often appear in the remaining part of this work.

For $k \in \mathbb{N}$, and $s, q \in \mathbb{R}$ with $1<q<\infty$, we define the weighted Sobolev spaces $W^{k, q, s}(G)$ by

$$
W^{k, q, s}(G)=\left\{f\left|\left(1+|x|^{2}\right)^{s / 2} \partial^{\alpha} f \in L^{q}(G),\right| \alpha \mid \leqslant k\right\},
$$

and we set $L^{q, s}(G)=W^{0, q, s}(G)$. For $\varphi \in W^{1, q}(G)$ we denote by $D(\varphi)$ the associated strain field defined by

$$
D(\varphi)_{i j}=\frac{1}{2}\left(\frac{\partial \varphi_{i}}{\partial x_{j}}+\frac{\partial \varphi_{j}}{\partial x_{i}}\right) \quad(i, j \in\{1,2,3\})
$$

To end this section, we recall several results due to Borchers and Sohr [2] and Iwashita [13], on the Stokes system in the exterior domain $E=\mathbb{R}^{3} \backslash \overline{\mathcal{O}}$, where $\mathcal{O} \subset \mathbb{R}^{3}$ is an open bounded set with $\partial \mathcal{O}$ of class $C^{2}$. More precisely, we consider the stationary Stokes problem:

$$
\begin{cases}\lambda v-\mu \Delta v+\nabla p=f & (x \in E), \\ \operatorname{div} v=0 & (x \in E), \\ v=0 & (x \in \partial \mathcal{O}) .\end{cases}
$$

By combining Theorem 1.2 in [2] and Corollary 3.2 in [13] we have: 
Theorem 2.2. Let $\theta \in\left(\frac{\pi}{2}, \pi\right)$ and let $\Sigma_{\theta}$ be the set defined in 2.1]. Then

1. Then there exist two families of operators $(R(\lambda))_{\lambda \in \Sigma_{\theta}}$ and $(P(\lambda))_{\lambda \in \Sigma_{\theta}}$ such that for every $\lambda \in \Sigma_{\theta}$ we have

$$
R(\lambda) \in \mathcal{L}\left(\left[L^{q}(E)\right]^{3},\left[W^{2, q}(E)\right]^{3}\right), \quad P(\lambda) \in \mathcal{L}\left(\left[L^{q}(E)\right]^{3}, \widehat{W}^{1, q}(E)\right), \quad(q>1),
$$

and the functions $v=R(\lambda) f$ and $p=P(\lambda) f$ satisfy 2.5. Moreover, there exists a positive constant $M$, depending only on $\mathcal{O}, q$ and $\theta$ such that for every $\lambda \in \Sigma_{\theta}$ we have

$$
|\lambda|\|R(\lambda) f\|_{q, E}+\|\mu \Delta R(\lambda) f-\nabla P(\lambda) f\|_{q, E} \leqslant M\|f\|_{q, E} \quad\left(q>1, f \in\left[L^{q}(E)\right]^{3}\right) .
$$

2. For every $q>1, \lambda \in \Sigma_{\theta}, m \in \mathbb{Z}_{+}, s>3\left(1-\frac{1}{q}\right)$ and $s^{\prime}<-\frac{3}{q}$, we have

$$
R(\lambda) \in \mathcal{L}\left(\left[W^{m, q, s}(E)\right]^{3},\left[W^{m+2, q, s^{\prime}}(E)\right]^{3}\right), \quad P(\lambda) \in \mathcal{L}\left(\left[W^{m, q, s}(E)\right]^{3}, W^{m+1, q, s^{\prime}}(E)\right) .
$$

Moreover, the functions $\lambda \mapsto R(\lambda)$ and $\lambda \mapsto P(\lambda)$ are holomorphic from $\Sigma_{\theta}$ to $\mathcal{L}\left(\left[W^{m, q, s}(E)\right]^{3},\left[W^{m+2, q, s^{\prime}}(E)\right]^{3}\right)$ and $\mathcal{L}\left(\left[W^{m, q, s}(E)\right]^{3}, W^{m+1, q, s^{\prime}}(E)\right)$, respectively. Finally, there exist

$$
R_{0} \in \mathcal{L}\left(\left[W^{m, q, s}(E)\right]^{3},\left[W^{m+2, q, s^{\prime}}(E)\right]^{3}\right), \quad P_{0} \in \mathcal{L}\left(\left[W^{m, q, s}(E)\right]^{3}, W^{m+1, q, s^{\prime}}(E)\right)
$$

such that

$$
\begin{gathered}
\limsup _{\lambda \in \Sigma_{\theta}, \lambda \rightarrow 0}|\lambda|^{-\frac{1}{2}}\left\|R(\lambda)-R_{0}\right\|_{\mathcal{L}\left(\left[W^{m, q, s}(E)\right]^{3},\left[W^{m+2, q, s^{\prime}}(E)\right]^{3}\right)}<\infty, \\
\limsup _{\lambda \in \Sigma_{\theta}, \lambda \rightarrow 0}|\lambda|^{-\frac{1}{2}}\left\|P(\lambda)-P_{0}\right\|_{\mathcal{L}\left(\left[W^{m, q, s}(E)\right]^{3}, W^{m+1, q, s^{\prime}}(E)\right)}<\infty .
\end{gathered}
$$

Remark 2.3. Setting $R(0):=R_{0}$ and $P(0):=P_{0}$, estimates 2.7) and 2.8) imply that the functions $\lambda \mapsto R(\lambda)$ and $\lambda \mapsto P(\lambda)$ extend to continuous functions from $\Sigma_{\theta} \cup\{0\}$ to $\mathcal{L}\left(\left[W^{m, q, s}(E)\right]^{3},\left[W^{m+2, q, s^{\prime}}(E)\right]^{3}\right)$ and $\mathcal{L}\left(\left[W^{m, q, s}(E)\right]^{3}, W^{m+1, q, s^{\prime}}(E)\right)$, respectively.

\section{Some background on the fluid-structure operator}

\subsection{Definition and first properties}

In this section, we introduce the fluid-structure operator and the fluid-structure semigroup and we remind some of their properties, as established in the existing literature. For the remaining part of this section the notation $\Omega$ designs either an open, connected and bounded subset of $\mathbb{R}^{3}$, with $\partial \Omega$ of class $C^{2}$, or we have $\Omega=\mathbb{R}^{3}$. Let $\mathcal{O}$ be an open bounded set with smooth boundary such that $\overline{\mathcal{O}} \subset \Omega$ and such that 0 is its center of mass. We denote $E_{\Omega}=\Omega \backslash \overline{\mathcal{O}}$ and we set $E_{\mathbb{R}^{3}}:=E$. Moreover, we denote by $\nu$ the unit normal vector on $\partial \mathcal{O}$ oriented towards the interior of $\mathcal{O}$.

Reminding notation (2.4) for the tensor field $D$, we introduce the function space

$$
\mathbb{X}^{q}(\Omega)=\left\{\Phi \in\left[L_{\sigma}^{q}(\Omega)\right]^{3} \quad \mid \quad D(\Phi)=0 \text { in } \mathcal{O}\right\},
$$


associated to the sets $\Omega$ and $\mathcal{O}$, which plays an important role in this work. Note that, for every $q \in(1, \infty)$ the dual $\left(\mathbb{X}^{q}(\Omega)\right)^{*}$ of $\mathbb{X}^{q}(\Omega)$ can be identified with $\mathbb{X}^{q^{\prime}}(\Omega)$, where $\frac{1}{q}+\frac{1}{q^{\prime}}=1$, with the duality pairing

$$
\langle f, g\rangle_{\mathbb{X}^{q^{\prime}}(\Omega), \mathbb{X} \mathbb{X}^{q}(\Omega)}=\int_{\mathcal{O}} \rho f \cdot g \mathrm{~d} x+\int_{E_{\Omega}} f \cdot g \mathrm{~d} x \quad\left(f \in \mathbb{X}^{q^{\prime}}(\Omega), g \in \mathbb{X}^{q}(\Omega)\right),
$$

where $\rho$ is the constant density of the rigid body. Our notation is making explicit only the dependence of $\mathbb{X}^{q}$ on $\Omega$ since these spaces will be used later on for various $\Omega$ and with fixed $\mathcal{O}$. For $\Omega=\mathbb{R}^{3}$, we simply set

$$
\mathbb{X}^{q}:=\mathbb{X}^{q}\left(\mathbb{R}^{3}\right)
$$

Since every $\Phi$ in $\mathbb{X}^{q}(\Omega)$ satisfies $D(\Phi)=0$ in $\mathcal{O}$, there exists a unique couple $\left[\begin{array}{l}\ell \\ \omega\end{array}\right] \in \mathbb{C}^{3} \times \mathbb{C}^{3}$ such that

$$
\Phi(x)=\varphi(x) \mathbb{1}_{E_{\Omega}}(x)+(\ell+\omega \times x) \mathbb{1}_{\mathcal{O}}(x) \quad(x \in \Omega),
$$

where $\mathbb{1}_{U}$ stands for the characteristic function of the set $U$ (see for instance [24, Lemma 1.1]). We can thus use the identification:

$$
\begin{aligned}
& \mathbb{X}^{q}(\Omega) \simeq\left\{\left[\begin{array}{l}
\varphi \\
\ell \\
\omega
\end{array}\right] \in\left[L^{q}\left(E_{\Omega}\right)\right]^{3} \times \mathbb{C}^{3} \times \mathbb{C}^{3}, \text { with } \operatorname{div}(\varphi)=0 \text { in } E_{\Omega},\right. \\
& \varphi(x) \cdot \nu(x)=(\ell+\omega \times x) \cdot \nu(x) \text { for } x \in \partial \mathcal{O} \text { and } \varphi(x) \cdot \nu(x)=0 \text { for } x \in \partial \Omega\} .
\end{aligned}
$$

The two results below will allow us to precisely introduce the projection operator $\mathbb{P}_{q, \Omega}$ from $\left[L^{q}(\Omega)\right]^{3}$ onto $\mathbb{X}^{q}(\Omega)$ which will be used used in the following, and which will be denoted by $\mathbb{P}_{q}$ when $\Omega=\mathbb{R}^{3}$.

Proposition 3.1. Let $\mathcal{O}$ be an open bounded set of $\mathbb{R}^{3}$ with $\partial \mathcal{O}$ of class $C^{2}$. For $q>1$ let $G_{1}^{q}$ and $G_{2}^{q}$ be the spaces

$$
\begin{gathered}
G_{1}^{q}=\left\{u \in\left[L^{q}\left(\mathbb{R}^{3}\right)\right]^{3} \mid u=\nabla q_{1} \text { for some } q_{1} \in L_{\text {loc }}^{1}\left(\mathbb{R}^{3}\right)\right\}, \\
G_{2}^{q}=\left\{\begin{array}{c|c}
\operatorname{div} u=0 \text { in } \mathbb{R}^{3}, \quad u=\nabla q_{2} \text { in } E, \quad q_{2} \in L_{\text {loc }}^{1}\left(\mathbb{R}^{3}\right), \\
u \in\left[L^{q}\left(\mathbb{R}^{3}\right)\right]^{3} \mid \begin{array}{c}
u=\varphi \text { in } \mathcal{O} \text { with } \varphi \in\left[L^{q}(\mathcal{O})\right]^{3}, \\
\int_{\mathcal{O}} \varphi \mathrm{d} y=-\int_{\partial \mathcal{O}} q_{2} \nu \mathrm{d} \gamma, \quad \int_{\mathcal{O}} \varphi \times y \mathrm{~d} y=-\int_{\partial \mathcal{O}} q_{2} \nu \times y \mathrm{~d} \gamma
\end{array}
\end{array}\right\} .
\end{gathered}
$$

Then for every $u \in\left[L^{q}\left(\mathbb{R}^{3}\right)\right]^{3}$ there exists a unique triple $\left[\begin{array}{c}v \\ w_{1} \\ w_{2}\end{array}\right] \in \mathbb{X}^{q} \times G_{1}^{q} \times G_{2}^{q}$ with

$$
u=v+w_{1}+w_{2} .
$$

The map $u \mapsto v$, denoted $\mathbb{P}_{q}$, is a projection operator form $\left[L^{q}(\Omega)\right]^{3}$ onto $\mathbb{X}^{q}(\Omega)$. Moreover, the dual of the operator $\mathbb{P}_{q}$ is $\mathbb{P}_{q^{\prime}}$, where $\frac{1}{q}+\frac{1}{q^{\prime}}=1$.

For the proof of Proposition 3.1 we refer to Wang and Xin [27, Theorem 2.2]. 
Proposition 3.2. Let $\Omega \subset \mathbb{R}^{3}$ be an open bounded set with $\partial \Omega$ of class $C^{2}$. Let $\mathcal{O}$ be an open bounded set with $\partial \mathcal{O}$ of class $C^{2}$ such that $\overline{\mathcal{O}} \subset \Omega$. For $q>1$ let $G_{1}^{q}(\Omega)$ and $G_{2}^{q}(\Omega)$ be the spaces

$$
\begin{gathered}
G_{1}^{q}(\Omega)=\left\{u \in\left[L^{q}(\Omega)\right]^{3} \quad \mid u=\nabla q_{1} \text { for some } q_{1} \in W_{0}^{1, q}(\Omega)\right\}, \\
G_{2}^{q}(\Omega)=\left\{\begin{array}{c}
\operatorname{div} u=0 \text { in } \Omega, \quad u=\nabla q_{2} \text { in } E_{\Omega}, \quad q_{2} \in W^{1, q}\left(E_{\Omega}\right), \\
u \in\left[L^{q}(\Omega)\right]^{3} \mid \begin{array}{c}
u=\varphi \text { in } \mathcal{O} \text { with } \varphi \in\left[L^{q}(\mathcal{O})\right]^{3}, \\
\int_{\mathcal{O}} \varphi \mathrm{d} y=-\int_{\partial \mathcal{O}} q_{2} \nu \mathrm{d} \gamma, \quad \int_{\mathcal{O}} \varphi \times y \mathrm{~d} y=-\int_{\partial \mathcal{O}} q_{2} \nu \times y \mathrm{~d} \gamma
\end{array}
\end{array}\right.
\end{gathered}
$$

Then for every $u \in\left[L^{q}(\Omega)\right]^{3}$ there exists a unique triple $\left(v, w_{1}, w_{2}\right) \in \mathbb{X}^{q}(\Omega) \times G_{1}^{q}(\Omega) \times G_{2}^{q}(\Omega)$ with

$$
u=v+w_{1}+w_{2} .
$$

The map $u \mapsto v$, denoted $\mathbb{P}_{q, \Omega}$, is a projection operator form $\left[L^{q}(\Omega)\right]^{3}$ onto $\mathbb{X}^{q}(\Omega)$. Furthermore, the dual of the operator $\mathbb{P}_{q, \Omega}$ is $\mathbb{P}_{q^{\prime}, \Omega}$, where $\frac{1}{q}+\frac{1}{q^{\prime}}=1$.

The proof of Proposition 3.2 is similar to the proof of [27, Theorem 2.2]. However, for the sake of completeness we provide a short proof in Appendix A

The fluid-structure operator on $\Omega$ is the operator $\mathbb{A}_{q, \Omega}: \mathcal{D}\left(\mathbb{A}_{q, \Omega}\right) \rightarrow \mathbb{X}^{q}(\Omega)$ defined, for every $q>1$, by

$$
\begin{gathered}
\mathcal{D}\left(\mathbb{A}_{q, \Omega}\right)=\left\{\varphi \in\left[W_{0}^{1, q}(\Omega)\right]^{3} \cap \mathbb{X}^{q}(\Omega) \mid\right. \\
\left.\varphi_{\mid E_{\Omega}} \in\left[W^{2, q}\left(E_{\Omega}\right)\right]^{3}\right\}, \\
\mathbb{A}_{q, \Omega} \varphi=\mathbb{P}_{q, \Omega} \mathcal{A}_{q, \Omega} \varphi \quad\left(\varphi \in \mathcal{D}\left(\mathbb{A}_{q, \Omega}\right)\right),
\end{gathered}
$$

where $\mathbb{P}_{q, \Omega}$ is the projector introduced in Proposition 3.1 and the operator $\mathcal{A}_{q, \Omega}: \mathcal{D}\left(\mathcal{A}_{q, \Omega}\right) \rightarrow$ $\left[L^{q}(\Omega)\right]^{3}$ is defined by $\mathcal{D}\left(\mathcal{A}_{q, \Omega}\right)=\mathcal{D}\left(\mathbb{A}_{q, \Omega}\right)$ and for every $\varphi \in \mathcal{D}\left(\mathcal{A}_{q, \Omega}\right)$,

$$
\mathcal{A}_{q, \Omega} \varphi= \begin{cases}\mu \Delta \varphi & \text { in } E_{\Omega}, \\ -2 \mu m^{-1} \int_{\partial \mathcal{O}} D(\varphi) \nu \mathrm{d} \gamma-\left(2 \mu \mathcal{J}^{-1} \int_{\partial \mathcal{O}} y \times D(\varphi) \nu d \gamma\right) \times y & \text { in } \mathcal{O} .\end{cases}
$$

where $m$ and $J$ are given in terms of the constant density $\rho$ of the body by

$$
m=\int_{\mathcal{O}} \rho d x, \quad \mathcal{J}=\left(\mathcal{J}_{k, \ell}\right)_{k, \ell \in\{1,2,3\}} \text { with } \mathcal{J}_{k, \ell}=\int_{\mathcal{O}} \rho\left(\delta_{k, \ell}|x|^{2}-x_{k} x_{\ell}\right) d x .
$$

In the case $\Omega=\mathbb{R}^{3}$, the operators $\mathbb{P}_{q, \Omega}, \mathcal{A}_{q, \Omega}$ and $\mathbb{A}_{q, \Omega}$ are denoted by $\mathbb{P}_{q}, \mathcal{A}_{q}$ and $\mathbb{A}_{q}$, respectively and $\mathbb{A}_{q}: \mathcal{D}\left(\mathbb{A}_{q}\right) \rightarrow \mathbb{X}^{q}$ is defined, for every $q>1$, by

$$
\begin{gathered}
\mathcal{D}\left(\mathbb{A}_{q}\right)=\left\{\varphi \in\left[W^{1, q}\left(\mathbb{R}^{3}\right)\right]^{3} \cap \mathbb{X}^{q} \mid \varphi_{\mid E} \in\left[W^{2, q}(E)\right]^{3}\right\}, \\
\mathbb{A}_{q} \varphi=\mathbb{P}_{q} \mathcal{A}_{q} \varphi \quad\left(\varphi \in \mathcal{D}\left(\mathbb{A}_{q}\right)\right) .
\end{gathered}
$$

In the case $q=2$ and when $\mathcal{O}$ is a ball, the fluid-structure operator $\mathcal{A}_{q}$ has been introduced in Takahashi and Tucsnak [23, where it has been proven that this operator generates an analytic semigroup on $\mathbb{X}^{2}$. Later, Wang and Xin [27] proved that the operator $\mathbb{A}_{q}$ generates an analytic semigroup on $\mathbb{X}^{6 / 5} \cap \mathbb{X}^{q}$ if $q \geqslant 2$ and that if the solid is a ball in $\mathbb{R}^{3}$ the operator $\mathbb{A}_{q}$ generates an analytic semigroup (not necessarily bounded) on $\mathbb{X}^{2} \cap \mathbb{X}^{q}$ if $q \geqslant 6$. One of our main result improves 
the result of Wang and Xin [27. Actually, in Theorem 6.1 we will prove that $\mathbb{A}_{q}$ generates a bounded analytic semigroup on $\mathbb{X}^{q}$ for any $q>1$. Moreover, this result is true for bodies of arbitrary shape.

It is important for future use to rephrase the resolvent equation for $\mathbb{A}_{q, \Omega}$ in a form involving only PDEs and algebraic constraints. To this aim, for $\lambda \in \mathbb{C}$, we consider the system

$$
\begin{cases}\lambda v-\mu \Delta v+\nabla \pi=f & \left(x \in E_{\Omega}\right), \\ \operatorname{div} v=0 & \left(x \in E_{\Omega}\right), \\ v=0 & (x \in \partial \Omega), \\ v=\ell+\omega \times x & (x \in \partial \mathcal{O}), \\ m \lambda \ell=-\int_{\partial \mathcal{O}} \sigma(v, \pi) \nu \mathrm{d} s+f_{\ell}, & \\ \mathcal{J} \lambda \omega=-\int_{\partial \mathcal{O}} x \times \sigma(v, \pi) \nu \mathrm{d} s+f_{\omega} . & \end{cases}
$$

In the above system the unknowns are $v, \pi, \ell$ and $\omega$, whereas

$$
\sigma(v, \pi):=-\pi I+2 \nu D(v)
$$

By slightly adapting the methodology used in [22, 23] for the case $q=2$, it can be checked that we have the following equivalence:

Proposition 3.3. Let $\Omega \subset \mathbb{R}^{3}$ be an open, connected and bounded set with $\partial \Omega$ of class $C^{2}$ or $\Omega=\mathbb{R}^{3}$. Let $1<q<\infty$ and $\lambda \in \mathbb{C}$. Assume that $f \in\left[L^{q}\left(E_{\Omega}\right)\right]^{3}$ and $f_{\ell}, f_{\omega} \in \mathbb{C}^{3}$. Then $(v, \pi, \ell, \omega) \in\left[W^{2, q}\left(E_{\Omega}\right)\right]^{3} \times \widehat{W}^{1, q}\left(E_{\Omega}\right) \times \mathbb{C}^{3} \times \mathbb{C}^{3}$ satisfies 3.12 if

$$
\left(\lambda I-\mathbb{A}_{q, \Omega}\right) V=\mathbb{P}_{q, \Omega} F
$$

where

$$
V=v \mathbb{1}_{E_{\Omega}}+(\ell+\omega \times x) \mathbb{1}_{\mathcal{O}}, \quad F=\mathbb{P}_{q, \Omega}\left(f \mathbb{1}_{E_{\Omega}}+\left(m^{-1} f_{\ell}+\mathcal{J}^{-1} x \times f_{\omega}\right) \mathbb{1}_{\mathcal{O}}\right) .
$$

Conversely, assume that $F \in \mathbb{X}^{q}(\Omega)$ and $V \in \mathcal{D}\left(\mathbb{A}_{q, \Omega}\right)$ satisfy 3.13 . Then there exists $\pi \in$ $\widehat{W}^{1, q}\left(E_{\Omega}\right)$ such that $(v, \ell, \omega) \in\left[W^{2, q}\left(E_{\Omega}\right)\right]^{3} \times \mathbb{C}^{3} \times \mathbb{C}^{3}$ satisfies 3.12 where

$$
v=\left.V\right|_{E_{\Omega}}, \quad \ell=\frac{1}{m} \int_{\mathcal{O}} V \mathrm{~d} x, \quad \omega=-\mathcal{J}^{-1} \int_{\mathcal{O}} V \times x \mathrm{~d} x
$$

and

$$
f=\left.F\right|_{E_{\Omega}}, \quad f_{\ell}=\frac{1}{m} \int_{\mathcal{O}} F \mathrm{~d} x, \quad f_{\omega}=-\mathcal{J}^{-1} \int_{\mathcal{O}} F \times x \mathrm{~d} x
$$

\subsection{The fluid-structure semigroup on bounded domains}

In this subsection we assume that $\Omega$ is an open bounded set in $\mathbb{R}^{3}$ with boundary of class $C^{2}$. In this case the operator $\mathbb{A}_{q, \Omega}$ has been extensively studied in Maity and Tucsnak [18. In particular, by combining Theorem 1.3 and Theorem 4.1 from [18] we have:

Theorem 3.4. With the above notation, let $q>1$ and assume that $\Omega \subset \mathbb{R}^{3}$ is bounded, with $\partial \Omega$ of class $C^{2}$. Then the operator $\mathbb{A}_{q, \Omega}$, defined in (3.6)-(3.7), generates an analytic and exponentially stable $C^{0}$-semigroup, denoted $\mathbb{T}^{q, \Omega}=\left(\mathbb{T}_{t}^{q, \Omega}\right)_{t \geqslant 0}$, on $\mathbb{X}^{q}(\Omega)$.

The above result has the following consequence, which follows by standard analytic semigroups theory: 
Corollary 3.5. With the notation and under the assumptions in Theorem 3.4, for every $\theta \in\left(\frac{\pi}{2}, \pi\right)$ the exists a constant $M$, possibly depending on $q, \theta, \mathcal{O}$ and $\Omega$, such that

$$
(1+|\lambda|)\left\|\left(\lambda I-\mathbb{A}_{q, \Omega}\right)^{-1}\right\|_{\mathcal{L}\left(\mathbb{X}_{q}(\Omega)\right)}+\left\|\mathbb{A}_{q, \Omega}\left(\lambda I-\mathbb{A}_{q, \Omega}\right)^{-1}\right\|_{\mathcal{L}\left(\mathbb{X}_{q}(\Omega)\right)} \leqslant M \quad\left(\lambda \in \Sigma_{\theta} \cup\{0\}\right) .
$$

By combining Corollary 3.5 and Proposition 3.3 we obtain the following result:

Proposition 3.6. Let $\theta \in(\pi / 2, \pi), q \in(1, \infty)$ and assume that $\Omega \subset \mathbb{R}^{3}$ is bounded, with $\partial \Omega$ of class $C^{2}$. Then there exists a constant $C>0$, possibly depending on $\theta, q$, $\Omega$ and $\mathcal{O}$, such that for all $\lambda \in \Sigma_{\theta}, f \in\left[L^{q}\left(E_{\Omega}\right)\right]^{3}$ and $f_{\ell}, f_{\omega} \in \mathbb{C}^{3}$, there exists a unique solution $(v, \pi, \ell, \omega) \in$ $\left[W^{2, q}\left(E_{\Omega}\right)\right]^{3} \times \widehat{W}^{1, q}\left(E_{\Omega}\right) \times \mathbb{C}^{3} \times \mathbb{C}^{3}$ of 3.12 satisfying

$$
(1+|\lambda|)\left(\|v\|_{q, E_{\Omega}}+|\ell|+|\omega|\right)+\|v\|_{2, q, E_{\Omega}}+\|\nabla \pi\|_{q, E_{\Omega}} \leqslant C\left(\|f\|_{q, E_{\Omega}}+\left|f_{\ell}\right|+\left|f_{\omega}\right|\right) .
$$

We need below the following slight generalization of Proposition 3.6

Corollary 3.7. With the notation and assumptions in Proposition 3.6, let $v \in\left[W^{2, q}\left(E_{\Omega}\right)\right]^{3}$, $\pi \in \widehat{W}^{1, q}\left(E_{\Omega}\right), \ell, \omega \in \mathbb{C}^{3}$ be such that

$$
\begin{array}{ll}
v(x)=0 & (x \in \partial \Omega) \\
v=\ell+\omega \times x & (x \in \partial \mathcal{O}),
\end{array}
$$

$\operatorname{div} v \in W_{0}^{1, q}\left(E_{\Omega}\right), \quad \int_{E_{\Omega}} \operatorname{div} v \mathrm{~d} x=0$.

Then for every $\lambda_{0}>0$ there exists a constant $C=C\left(\Omega, p, \lambda_{0}, \theta\right)$ such that

$$
\begin{aligned}
|\lambda|\left(\|v\|_{q, E_{\Omega}}+|\ell|+|\omega|\right)+ & \left\|D^{2} v\right\|_{q, E_{\Omega}}+\|\nabla \pi\|_{q, E_{\Omega}} \\
\leqslant & C\left(\|\lambda v-\Delta v+\nabla \pi\|_{q, E_{\Omega}}+\|\nabla \operatorname{div} v\|_{q, E_{\Omega}}\right. \\
& \left.+\left|m \lambda \ell+\int_{\partial \mathcal{O}} \sigma(v, \pi) \nu \mathrm{d} s\right|+\left|\mathcal{J} \lambda \omega+\int_{\partial \mathcal{O}} x \times \sigma(v, \pi) \nu \mathrm{d} s\right|\right),
\end{aligned}
$$

for every $\lambda \in \Sigma_{\theta}$ with $|\lambda| \leqslant \lambda_{0}$.

Proof. According to Lemma 2.1 there exists $\tilde{v} \in\left[W_{0}^{2, q}\left(E_{\Omega}\right)\right]^{3}$ such that $\operatorname{div} \tilde{v}=\operatorname{div} v$ on $E_{\Omega}$ and

$$
\|\tilde{v}\|_{2, q, E_{\Omega}} \leqslant C\|\operatorname{div} v\|_{1, q, E_{\Omega}},
$$

where $C$ is a constant depending only on $\Omega$ and on $q$. Setting $u=v-\tilde{v}$ we see that $u \in\left[W^{2, q}\left(E_{\Omega}\right)\right]^{3}$ and

$$
\begin{array}{ll}
u(x)=0 & (x \in \partial \Omega), \\
u(x)=\ell+\omega \times x & (x \in \partial \mathcal{O}), \\
\sigma(u, \pi)=\sigma(v, \pi) & (x \in \partial \mathcal{O}), \\
\operatorname{div} u=0 & \left(x \in E_{\Omega}\right) .
\end{array}
$$

By applying Proposition 3.6 and elementary inequalities, it follows that

$$
\begin{aligned}
|\lambda|\left(\|u\|_{q, E_{\Omega}}\right. & +|\ell|+|\omega|)+\left\|D^{2} u\right\|_{q, E_{\Omega}}+\|\nabla \pi\|_{q, E_{\Omega}} \leqslant C\|\lambda u-\mu \Delta u+\nabla \pi\|_{q, E_{\Omega}} \\
& +C\left|m \lambda \ell+\int_{\partial \mathcal{O}} \sigma(v, \pi) \nu \mathrm{d} s\right|+C\left|\mathcal{J} \lambda \omega+\int_{\partial \mathcal{O}} x \times \sigma(v, \pi) \nu \mathrm{d} s\right| \quad\left(\lambda \in \Sigma_{\theta}\right) .
\end{aligned}
$$

The above estimate and 3.16 imply the conclusion 3.15). 


\section{From the Stokes operator in exterior domains to the fluid- structure operator in the whole space}

In this section, we study the fluid structure operator $\mathbb{A}_{q, \Omega}$, defined in $(3.10)-(3.11)$, in the case $\Omega=\mathbb{R}^{3}$. As mentioned in Sections 2 and 3 in this case the space $\mathbb{X}^{q}(\Omega)$, defined in $(3.1)$, and the operators $\mathbb{P}_{q, \Omega}, \mathbb{A}_{q, \Omega}$ are simply denoted by $\mathbb{X}^{q}, \mathbb{P}_{q}$ and $\mathbb{A}_{q}$, respectively. The main idea developed in this section is that the resolvent of the fluid-structure operator can be expressed in terms of the resolvent of the Stokes operator with homogeneous Dirichlet conditions on the boundary of an obstacle of arbitrary shape $\mathcal{O}$. The connection between these two families of resolvents is then used to study the behaviour of the of $\left(\lambda I-\mathbb{A}_{q}\right)^{-1}$ for $\lambda$ close to zero, in the spirit of the similar results for the Stokes operator in exterior domains obtained by Iwashita [13.

Let $\mathcal{O}$ be an open, bounded subset of $\mathbb{R}^{3}$ with $\partial \mathcal{O}$ of class $C^{2}$ and let $E=\mathbb{R}^{3} \backslash \mathcal{O}$. We consider the system

$$
\begin{cases}\lambda u-\mu \Delta u+\nabla \pi=f & (x \in E), \\ \operatorname{div} u=0 & (x \in E), \\ u=\ell+\omega \times x & (x \in \partial \mathcal{O}), \\ m \lambda \ell=-\int_{\partial \mathcal{O}} \sigma(u, \pi) \nu \mathrm{d} s+f_{\ell}, & \\ \mathcal{J} \lambda \omega=-\int_{\partial \mathcal{O}} x \times \sigma(u, \pi) \nu \mathrm{d} s+f_{\omega}, & \end{cases}
$$

where $f \in\left[L^{q}(E)\right]^{3}, f_{\ell}, f_{\omega} \in \mathbb{C}^{3}$ and $\lambda \in \mathbb{C}$. In the above system the unknowns are $u, \pi, \ell$ and $\omega$, whereas

$$
\sigma(u, \pi):=-\pi I+2 \mu D(u)
$$

To study the solvability of 4.1 we introduce several auxiliary operators.

Firstly, given $\lambda \in \mathbb{C}$ and $\ell, \omega \in \mathbb{C}^{3}$, we consider the boundary value problem:

$$
\left\{\begin{array}{lr}
\lambda w-\mu \Delta w+\nabla \eta=0, \quad \operatorname{div} w=0 & (x \in E), \\
w(x)=\ell+\omega \times x & (x \in \partial \mathcal{O}),
\end{array}\right.
$$

and we remind the notation 2.3 (and more generally the notation in Section 2 for the possibly weighted Sobolev spaces in unbounded domains.

Proposition 4.1. Assume that $\theta \in(0, \pi)$. Then for all $q>1$, for every $\lambda \in \Sigma_{\theta}$ and $\ell, \omega \in \mathbb{C}^{3}$, the system 4.2 admits a unique solution $(w, \eta) \in\left[W^{2, q}(E)\right]^{3} \times \widehat{W}^{1, q}(E)$. Moreover, let $\left(D_{\lambda}\right)_{\lambda \in \Sigma_{\theta}}$ be the family of operators defined by

$$
D_{\lambda}\left[\begin{array}{l}
\ell \\
\omega
\end{array}\right]=\left[\begin{array}{l}
w \\
\eta
\end{array}\right] \quad\left(\lambda \in \Sigma_{\theta}, \ell, \omega \in \mathbb{C}^{3}\right),
$$

where $(w, \eta) \in\left[W^{2, q}(E)\right]^{3} \times \widehat{W}^{1, q}(E)$ is the solution of 4.2 . Then for every $\lambda \in \Sigma_{\theta}$ and $m \in \mathbb{N}$, we have

$$
\begin{gathered}
D_{\lambda} \in \mathcal{L}\left(\mathbb{C}^{6},\left[W^{m+1, q}(E)\right]^{3} \times \widehat{W}^{m, q}(E)\right), \\
D_{\lambda} \in \mathcal{L}\left(\mathbb{C}^{6},\left[W^{m+1, q, s^{\prime}}(E)\right]^{3} \times W^{m, q, s^{\prime}}(E)\right) \quad\left(s^{\prime}<-\frac{3}{q}\right) .
\end{gathered}
$$

Finally, there exists

$$
D_{0} \in \bigcap_{m \in \mathbb{N}, q>1, s^{\prime}<-\frac{3}{q}} \mathcal{L}\left(\mathbb{C}^{6},\left[W^{m+1, q, s^{\prime}}(E)\right]^{3} \times W^{m, q, s^{\prime}}(E)\right)
$$


such that

$$
\limsup _{\lambda \in \Sigma_{\theta}, \lambda \rightarrow 0}|\lambda|^{-\frac{1}{2}}\left\|D(\lambda)-D_{0}\right\|_{\mathcal{L}\left(\mathbb{C}^{6},\left[W^{m+1, q, s^{\prime}}(E)\right]^{3} \times W^{m, q, s^{\prime}}(E)\right)}<\infty,
$$

for every $m \in \mathbb{N}, q>1$ and $s^{\prime}<-\frac{3}{q}$.

Proof. We choose two balls $B_{1}$ and $B_{2}$ in $\mathbb{R}^{3}$ such that $\overline{\mathcal{O}} \subset B_{1} \subset \bar{B}_{1} \subset B_{2}$. We define a cut-off function $\chi \in C^{\infty}\left(\mathbb{R}^{3}\right)$ such that $\chi(x) \in[0,1]$ for every $x \in \mathbb{R}^{3}$ and

$$
\chi(x)= \begin{cases}1 & \text { if } x \in \bar{B}_{1}, \\ 0 & \text { if } x \in E \backslash B_{2} .\end{cases}
$$

We set

$$
\bar{w}(x)=\chi(x)(\ell+\omega \times x)-\mathbb{B}_{B_{2} \backslash \bar{B}_{1}}(\nabla \chi \cdot(\ell+\omega \times x)),
$$

where $\mathbb{B}_{B_{2} \backslash \bar{B}_{1}}$ is the Bogovskii operator as introduced in Lemma 2.1. It is easy to see that, div $\bar{w}=0$ in $E, \bar{w}(x)=\ell+\omega \times x$ for $x \in \partial E$ and $\bar{w} \in W^{k, q}(E)$, for any $k \in \mathbb{N}$. Since $w=\widetilde{w}+\bar{w}$, where $\widetilde{w}$ satisfies

$$
\left\{\begin{array}{lll}
\lambda \widetilde{w}-\mu \Delta \widetilde{w}+\nabla \eta=-\lambda \bar{w}+\nu \Delta \bar{w}, & \operatorname{div} \widetilde{w}=0 & (x \in E), \\
\widetilde{w}=0 & (x \in \partial \mathcal{O}) .
\end{array}\right.
$$

We can apply classical regularity results for Stokes (e.g. [13, Proposition 2.7(i)]) to get 4.4 and Theorem 2.2 to obtain (4.5)- 4.6).

The above result allows us to introduce the family of operators $\left(\mathcal{T}_{\lambda}\right)_{\lambda \in \Sigma_{\theta}} \subset \mathcal{L}\left(\mathbb{C}^{6}\right)$ defined by

$$
\mathcal{T}_{\lambda}\left[\begin{array}{c}
\ell \\
\omega
\end{array}\right]=\left[\begin{array}{c}
\int_{\partial \mathcal{O}} \sigma(w, \eta) \nu \mathrm{d} s \\
\int_{\partial \mathcal{O}} x \times \sigma(w, \eta) \nu \mathrm{d} s
\end{array}\right] \quad\left(\lambda \in \Sigma_{\theta}, \ell, \omega \in \mathbb{C}^{3}\right)
$$

where $(w, \eta)$ is the solution of 4.2 , given by $D_{\lambda}$ according to 4.3 .

Proposition 4.2. Let $\theta \in(0, \pi)$. For every $\lambda \in \Sigma_{\theta}$ let $\left(\mathcal{T}_{\lambda}\right)_{\lambda \in \Sigma_{\theta}}$ be the operators defined in (4.7) and let $\left(\mathcal{K}_{\lambda}\right)_{\lambda \in \Sigma_{\theta}}$ be the family of operators defined by

$$
\mathcal{K}_{\lambda}=\left[\begin{array}{cc}
\lambda m I_{3} & 0 \\
0 & \lambda \mathcal{J}
\end{array}\right]+\mathcal{T}_{\lambda} \quad\left(\lambda \in \Sigma_{\theta}\right)
$$

Then there exists $\mathcal{K}_{0} \in \mathcal{L}\left(\mathbb{C}^{6}\right)$ invertible such that

$$
\limsup _{\lambda \in \Sigma_{\theta}, \lambda \rightarrow 0}|\lambda|^{-\frac{1}{2}}\left\|\mathcal{K}_{\lambda}-\mathcal{K}_{0}\right\|_{\mathcal{L}\left(\mathbb{C}^{6}\right)}<\infty .
$$

Moreover, $\mathcal{K}_{\lambda}$ is invertible for every $\lambda \in \Sigma_{\theta}$ and

$$
\limsup _{\lambda \in \Sigma_{\theta}, \lambda \rightarrow 0}|\lambda|^{-\frac{1}{2}}\left\|\mathcal{K}_{\lambda}^{-1}-\mathcal{K}_{0}^{-1}\right\|_{\mathcal{L}\left(\mathbb{C}^{6}\right)}<\infty .
$$

Proof. For $\ell, \omega \in \mathbb{C}^{3}$ we set

$$
\left[\begin{array}{c}
w_{0} \\
\eta_{0}
\end{array}\right]=D_{0}\left[\begin{array}{l}
\ell \\
\omega
\end{array}\right], \quad \mathcal{K}_{0}\left[\begin{array}{l}
\ell \\
\omega
\end{array}\right]=\left[\begin{array}{c}
\int_{\partial \mathcal{O}} \sigma\left(w_{0}, \eta_{0}\right) \nu \mathrm{d} s \\
\left.\int_{\partial \mathcal{O}} x \times \sigma\left(w_{0}, \eta_{0}\right) \nu \mathrm{d} s\right]
\end{array}\right]
$$

where $D_{0}$ is the operator introduced in Proposition 4.1. Applying Proposition 4.1 and a standard trace theorem it follows that 4.9 ) holds. The fact that $\mathcal{K}_{0}$ (which is called the resistance matrix of 
$\mathcal{O}$ ) is invertible is a classical result (see, for instance, Happel and Brenner [9, Section 5.4], where it is shown that this matrix is strictly positive).

On the other hand, taking the inner product in $\left[L^{2}(E)\right]^{3}$ of the first equation in 4.2 by $w$, integrating by parts and using the second equation in 4.2 it follows that

$$
\left\langle\mathcal{T}_{\lambda}\left[\begin{array}{l}
\ell \\
\omega
\end{array}\right],\left[\begin{array}{l}
\ell \\
\omega
\end{array}\right]\right\rangle_{\mathbb{C}^{6}}=\lambda \int_{E}|w|^{2} \mathrm{~d} x+\mu \int_{E}|D(w)|^{2} \mathrm{~d} x \quad\left(\ell, \omega \in \mathbb{C}^{3}, \lambda \in \Sigma_{\theta}\right) .
$$

Assume now that $\ell, \omega \in \mathbb{C}^{3}$ and $\lambda \in \Sigma_{\theta}$ are such that

$$
\mathcal{K}_{\lambda}\left[\begin{array}{l}
\ell \\
\omega
\end{array}\right]=\left[\begin{array}{cc}
\lambda m I_{3} & 0 \\
0 & \lambda \mathcal{J}
\end{array}\right]\left[\begin{array}{l}
\ell \\
\omega
\end{array}\right]+\mathcal{T}_{\lambda}\left[\begin{array}{l}
\ell \\
\omega
\end{array}\right]=0
$$

Taking the inner product in $\mathbb{C}^{6}$ of the two sides of the above formula by $\left[\begin{array}{l}\ell \\ \omega\end{array}\right]$ and using 4.11 it follows that

$$
\lambda m|\ell|^{2}+\lambda\langle\mathcal{J} \omega, \omega\rangle_{\mathbb{C}^{3}}+\lambda \int_{E}|w|^{2} \mathrm{~d} x+\mu \int_{E}|D(w)|^{2}=0 .
$$

If $\lambda \in \Sigma_{\theta}$ with $\operatorname{Im} \lambda \neq 0$ it follows that $\ell=0$ and $\omega=0$. On the other hand, if $\lambda \in \Sigma_{\theta}$ and $\operatorname{Im} \lambda=0$ we have $\operatorname{Re} \lambda>0$. In this case, we obtain $w=0$ and consequently $\ell=\omega=0$. We have thus shown that the operator in (4.8) is invertible for every $\lambda \in \Sigma_{\theta}$. This fact, (4.9) and the fact that $\mathcal{K}_{0}$ is invertible finally imply 4.10 .

We are now in a position to state the main result in this section.

Theorem 4.3. Let $q \in(1, \infty)$ and $\theta \in\left(\frac{\pi}{2}, \pi\right)$. Then

1. For every $\lambda \in \Sigma_{\theta}$ there exist operators

$$
\mathcal{R}(\lambda) \in \mathcal{L}\left(\left[L^{q}(E)\right]^{3} \times \mathbb{C}^{6},\left[W^{2, q}(E)\right]^{3} \times \mathbb{C}^{6}\right), \quad \mathcal{P}(\lambda) \in \mathcal{L}\left(\left[L^{q}(E)\right]^{3} \times \mathbb{C}^{6}, \widehat{W}^{1, q}(E)\right),
$$

such that, for $f \in\left[L^{q}(E)\right]^{3}, f_{\ell}, f_{\omega} \in \mathbb{C}^{3}$, setting

$$
\left[\begin{array}{c}
u \\
\ell \\
\omega
\end{array}\right]=\mathcal{R}(\lambda)\left[\begin{array}{c}
f \\
f_{\ell} \\
f_{\omega}
\end{array}\right], \quad \pi=\mathcal{P}(\lambda)\left[\begin{array}{c}
f \\
f_{\ell} \\
f_{\omega}
\end{array}\right]
$$

then $u, \ell, \omega$ and $\pi$ satisfy 4.1.

2. For $\lambda \in \Sigma_{\theta}, m \in \mathbb{Z}_{+}, s>3\left(1-\frac{1}{q}\right)$ and $s^{\prime}<-\frac{3}{q}$, we have

$$
\begin{gathered}
\mathcal{R}(\lambda) \in \mathcal{L}\left(\left[W^{m, q, s}(E)\right]^{3} \times \mathbb{C}^{6},\left[W^{m+2, q, s^{\prime}}(E)\right]^{3} \times \mathbb{C}^{6}\right), \\
\mathcal{P}(\lambda) \in \mathcal{L}\left(\left[W^{m, q, s}(E)\right]^{3} \times \mathbb{C}^{6}, W^{m+1, q, s^{\prime}}(E)\right) .
\end{gathered}
$$

Moreover, the functions $\lambda \mapsto \mathcal{R}(\lambda)$ and $\lambda \mapsto \mathcal{P}(\lambda)$ are holomorphic from $\Sigma_{\theta}$ to $\mathcal{L}\left(\left[W^{m, q, s}(E)\right]^{3} \times \mathbb{C}^{6},\left[W^{m+2, q, s^{\prime}}(E)\right]^{3} \times \mathbb{C}^{6}\right)$ and $\mathcal{L}\left(\left[W^{m, q, s}(E)\right]^{3} \times \mathbb{C}^{6}, W^{m+1, q, s^{\prime}}(E)\right), r e-$ spectively. Finally, there exist

$$
\begin{aligned}
& \mathcal{R}_{0} \in \mathcal{L}\left(\left[W^{m, q, s}(E)\right]^{3} \times \mathbb{C}^{6},\left[W^{m+2, q, s^{\prime}}(E)\right]^{3} \times \mathbb{C}^{6}\right), \\
& \mathcal{P}_{0} \in \mathcal{L}\left(\left[W^{m, q, s}(E)\right]^{3} \times \mathbb{C}^{6}, W^{m+1, q, s^{\prime}}(E)\right)
\end{aligned}
$$


such that

$$
\begin{array}{r}
\limsup _{\lambda \in \Sigma_{\theta}, \lambda \rightarrow 0}|\lambda|^{-\frac{1}{2}}\left\|\mathcal{R}(\lambda)-\mathcal{R}_{0}\right\|_{\mathcal{L}\left(\left[W^{m, q, s}(E)\right]^{3} \times \mathbb{C}^{6},\left[W^{m+2, q, s^{\prime}}(E) \times \mathbb{C}^{6}\right]^{3}\right)}<\infty \\
\lim _{\lambda \in \Sigma_{\theta}, \lambda \rightarrow 0}|\lambda|^{-\frac{1}{2}}\left\|\mathcal{P}(\lambda)-\mathcal{P}_{0}\right\|_{\mathcal{L}\left(\left[W^{m, q, s}(E)\right]^{3} \times \mathbb{C}^{6}, W^{m+1, q, s^{\prime}}(E)\right)}<\infty .
\end{array}
$$

Proof. Let $m \in \mathbb{Z}_{+}, q>1, s>0, f \in\left[W^{m, q, s}(E)\right]^{3}$ and $f_{\ell}, f_{\omega} \in \mathbb{C}^{3}$. For $\lambda \in \Sigma_{\theta} \cup\{0\}$ we remind from Proposition 4.2 that the matrix $\mathcal{K}_{\lambda}$, defined in 4.8, is invertible and we set

$$
\left[\begin{array}{l}
\ell_{\lambda} \\
\omega_{\lambda}
\end{array}\right]=\mathcal{K}_{\lambda}^{-1}\left[\begin{array}{c}
f_{\ell}-\int_{\partial \mathcal{O}} \sigma(R(\lambda) f, P(\lambda) f) \nu \mathrm{d} s \\
f_{\omega}-\int_{\partial \mathcal{O}} x \times \sigma(R(\lambda) f, P(\lambda) f) \nu \mathrm{d} s
\end{array}\right] \quad\left(\lambda \in \Sigma_{\theta} \cup\{0\}\right),
$$

where $(R(\lambda))$ and $(P(\lambda))$ are the families of operators introduced in Theorem 2.2 and Remark 2.3 . The last formula implies, according to Proposition 4.2 and Theorem 2.2 , that there exist $\delta, c_{\delta}>0$ such that

$$
\left|\ell_{\lambda}\right|+\left|\omega_{\lambda}\right| \leqslant c_{\delta}\left(\left|f_{\ell}\right|+\left|f_{\omega}\right|+\|f\|_{\left[W^{m, q, s}(E)\right]^{3}}\right) \quad\left(\lambda \in \Sigma_{\theta} \cup\{0\},|\lambda| \leqslant \delta\right) .
$$

For $\lambda \in \Sigma_{\theta} \cup\{0\}$ we set $\left[\begin{array}{l}v_{\lambda} \\ \eta_{\lambda}\end{array}\right]=D_{\lambda}\left[\begin{array}{l}\ell_{\lambda} \\ \omega_{\lambda}\end{array}\right]$, where $\left(D_{\lambda}\right)_{\lambda \in \Sigma_{\theta} \cup\{0\}}$ is the family of operators introduced in Proposition 4.1, and we define

$$
\left[\begin{array}{l}
u_{\lambda} \\
\pi_{\lambda}
\end{array}\right]=\left[\begin{array}{l}
R(\lambda) f \\
P(\lambda) f
\end{array}\right]+\left[\begin{array}{l}
v_{\lambda} \\
\eta_{\lambda}
\end{array}\right] \quad\left(\lambda \in \Sigma_{\theta} \cup\{0\}\right)
$$

where the operators $(R(\lambda))_{\lambda \in \Sigma_{\theta} \cup\{0\}},(P(\lambda))_{\lambda \in \Sigma_{\theta} \cup\{0\}}$ have been introduced in Theorem 2.2 and Remark 2.3. By combining Theorem 2.2 Proposition 4.1 and 4.18 it follows that for every $s>3\left(1-\frac{1}{q}\right), s^{\prime}<-\frac{3}{q}$ and $\delta>0$ there exists $d>0$ (possibly depending on $s, s^{\prime}$ and $\delta$ ) such that

$$
\begin{array}{r}
\left\|u_{\lambda}\right\|_{\left[W^{m+2, q, s^{\prime}}(E)\right]^{3}+\left\|\pi_{\lambda}\right\|_{W^{m+1, q, s^{\prime}}(E)} \leqslant d\left(\left|f_{\ell}\right|+\left|f_{\omega}\right|+\|f\|_{\left[W^{m, q, s}(E)\right]^{3}}\right)} \\
\left(\lambda \in \Sigma_{\theta} \cup\{0\},|\lambda| \leqslant \delta, f \in\left[W^{m, q, s}(E)\right]^{3}, f_{\ell}, f_{\omega} \in \mathbb{C}^{3}\right) .
\end{array}
$$

By combining 4.17) and 4.19 it follows that for every $\lambda \in \Sigma_{\theta}$ we have that $u=u_{\lambda}, \ell=\ell_{\lambda}$, $\omega=\omega_{\lambda}$ and $\pi=\pi_{\lambda}$ satisfy $(4.1)$. Consequently, if we set

$$
\begin{aligned}
\mathcal{R}(\lambda)\left[\begin{array}{c}
f \\
f_{\ell} \\
f_{\omega}
\end{array}\right] & =\left[\begin{array}{c}
R(\lambda) f+v_{\lambda} \\
\ell_{\lambda} \\
\omega_{\lambda}
\end{array}\right] \quad\left(\lambda \in \Sigma_{\theta} \cup\{0\}\right), \\
\mathcal{P}(\lambda)\left[\begin{array}{c}
f \\
f_{\ell} \\
f_{\omega}
\end{array}\right] & =P(\lambda) f+\eta_{\lambda}, \quad\left(\lambda \in \Sigma_{\theta} \cup\{0\}\right),
\end{aligned}
$$

then for every $\lambda \in \Sigma_{\theta}$ the operators $\mathcal{R}(\lambda), \mathcal{P}(\lambda)$ satisfy 4.13), 4.14 and $u, \ell, \omega$ and $\pi$ defined by 4.12 is indeed a solution of (4.1).

Finally the properties 4.15) and 4.16), with $\mathcal{R}_{0}:=\mathcal{R}(0)$, follow now from 4.21, 4.22, together with 4.18 and 4.20). 


\section{Further properties of the fluid-structure semigroup in $\mathbb{R}^{3}$}

In this section we study the fluid structure operator $\mathbb{A}_{q, \Omega}$, defined in $3.10-(3.11)$, in the case $\Omega=\mathbb{R}^{3}$. More precisely, we give several results opening the way to the proofs of the facts that $\mathbb{A}_{q}$ generates a bounded analytic semigroup and of the decay estimates for the fluid-structure operator by collecting several results which follow quite easily from the existing literature. The first one is:

Proposition 5.1. Let $1<q<\infty$ and let $\theta \in\left(\frac{\pi}{2}, \pi\right)$. Then there exist $\gamma>0$ and $m_{q, \theta}>0$ such that

$$
\left\|\lambda\left(\lambda I-\mathbb{A}_{q}\right)^{-1}\right\|_{\mathcal{L}\left(\mathbb{X}^{q}\right)} \leqslant m_{q, \theta}, \quad\left(\lambda \in \Sigma_{\theta},|\lambda| \geqslant \gamma\right) .
$$

Consequently, $\mathbb{A}_{q}$ generates an analytic semigroup on $\mathbb{X}^{q}$.

The proof of the above result can be obtained by a perturbation argument. Since this argument is a slight variation of the proof of Theorem 3.1 in [18, where the similar estimate is detailed for the case of fluid-structure system confined in a bounded domain, we omit the proof. We also note that by combining Proposition 3.3 and the first statement of Theorem 4.3 , we have

Proposition 5.2. For every $\lambda \in \Sigma_{\theta}$ and $F \in \mathbb{X}^{q}$, setting

$$
\mathcal{R}(\lambda)\left[\begin{array}{c}
\left.F\right|_{E} \\
\ell_{F} \\
\omega_{F}
\end{array}\right]=\left[\begin{array}{c}
u_{\lambda, F} \\
\ell_{\lambda, F} \\
\omega_{\lambda, F}
\end{array}\right]
$$

where the family $(\mathcal{R}(\lambda))$ has been introduced in 4.3 and

$$
\ell_{F}=\frac{1}{m} \int_{\mathcal{O}} F \mathrm{~d} x, \quad \omega_{F}=-\mathcal{J}^{-1} \int_{\mathcal{O}} F \times x \mathrm{~d} x
$$

we have

$$
\left(\lambda I-\mathbb{A}_{q}\right)^{-1} F=u_{\lambda, F} \mathbb{1}_{E}+\left(\ell_{\lambda, F}+\omega_{\lambda, F} \times x\right) \mathbb{1}_{\mathcal{O}} .
$$

The result below provides some simple but important properties of the fluid-structure operator $\mathbb{A}_{q}$.

Proposition 5.3. For every $1<q<\infty$, the dual $\mathbb{A}_{q}^{*}$ of $\mathbb{A}_{q}$ is given by $\mathbb{A}_{q}^{*}=\mathbb{A}_{q^{\prime}}$, with $\frac{1}{q}+\frac{1}{q^{\prime}}=1$.

Proof. For $G \in \mathbb{X}^{q^{\prime}}$, we set

$$
\ell_{G}=\frac{1}{m} \int_{\mathcal{O}} G \mathrm{~d} x, \quad \omega_{G}=-\mathcal{J}^{-1} \int_{\mathcal{O}} G \times x \mathrm{~d} x .
$$

We consider the equation

$$
\left(\lambda I-\mathbb{A}_{q^{\prime}}\right) W=G,
$$

which according to Proposition 3.3 is equivalent to the system

$$
\begin{cases}\lambda \varphi-\operatorname{div} \sigma\left(\varphi, \pi_{\varphi}\right)=\left.G\right|_{E}, \quad \operatorname{div} \varphi=0 & (x \in E), \\ \varphi=\psi+\kappa \times x, & (x \in \partial \mathcal{O}), \\ \lambda m \psi=-\int_{\Gamma} \sigma\left(\varphi, \pi_{\varphi}\right) \nu \mathrm{d} s+\ell_{G}, & \\ \lambda \mathcal{J} \kappa=-\int_{\Gamma} y \times \sigma\left(\varphi, \pi_{\varphi}\right) \nu \mathrm{d} s+\omega_{G}, & \end{cases}
$$

where

$$
\varphi=\left.W\right|_{E}, \quad \psi=\frac{1}{m} \int_{\mathcal{O}} W \mathrm{~d} x, \quad \kappa=-\mathcal{J}^{-1} \int_{\mathcal{O}} W \times x \mathrm{~d} x .
$$


Assume that $u \in\left[W^{2, q}(E)\right]^{3}, \pi \in \widehat{W}^{1, q}(E), \ell \in \mathbb{C}^{3}$ and $\omega \in \mathbb{C}^{3}$ satisfy the system (4.1). Taking the inner product in $\mathbb{C}^{3}$, of $(5.6)$ by $u$ and of $(4.1)$ by $\varphi$, integrating by parts and summing up the two formulas we obtain

$$
\int_{E}\langle f, \varphi\rangle_{\mathbb{C}^{3}} \mathrm{~d} x+\int_{\mathcal{O}} \sigma(u, \pi) \nu \cdot \varphi \mathrm{d} s=\int_{E}\langle u, G\rangle_{\mathbb{C}^{3}} \mathrm{~d} x+\int_{\mathcal{O}} \sigma\left(\varphi, \pi_{\varphi}\right) \nu \cdot u \mathrm{~d} s .
$$

Using the boundary conditions, the above relation can be written as

$$
\int_{E}\langle f, \varphi\rangle_{\mathbb{C}^{3}} \mathrm{~d} x+\left\langle f_{\ell}, \psi\right\rangle_{\mathbb{C}^{3}}+\left\langle f_{\omega}, \kappa\right\rangle_{\mathbb{C}^{3}}=\int_{E}\langle u, G\rangle_{\mathbb{C}^{3}} \mathrm{~d} x+\left\langle\ell, \ell_{G}\right\rangle_{\mathbb{C}^{3}}+\left\langle\omega, \omega_{G}\right\rangle_{\mathbb{C}^{3}} .
$$

In terms of the operator $\mathbb{A}_{q}$ and $\mathbb{A}_{q^{\prime}}$, the above equality reads as

$$
\left\langle\left(\lambda I-\mathbb{A}_{q}\right) U, W\right\rangle_{\mathbb{X}^{q}, \mathbb{X}^{q^{\prime}}}=\left\langle U,\left(\lambda I-\mathbb{A}_{q^{\prime}}\right) W\right\rangle_{\mathbb{X}^{q}, \mathbb{X}^{q^{\prime}}}, \quad\left(U \in \mathcal{D}\left(\mathbb{A}_{q}\right), W \in \mathcal{D}\left(\mathbb{A}_{q^{\prime}}\right)\right),
$$

with $U=u \mathbb{1}_{E}+(\ell+\omega \times y) \mathbb{1}_{\mathcal{O}}$. Therefore from the above identity we deduce $\mathcal{D}\left(\mathbb{A}_{q^{\prime}}\right) \subset \mathcal{D}\left(\mathbb{A}_{q}^{*}\right)$. In order to prove the reverse inclusion, we first note that, for $\lambda_{0}>0$ large enough the operator $\left(\lambda_{0} I-\mathbb{A}_{q^{\prime}}\right)$ is invertible (see Proposition 5.1). Take $\lambda_{0}$ as above and $W \in \mathcal{D}\left(\left(\lambda_{0} I-\mathbb{A}_{q}\right)^{*}\right)$. Since $\mathbb{X}_{q}^{*}=\mathbb{X}_{q^{\prime}}$, there exists $\widetilde{U} \in \mathcal{D}\left(\mathbb{A}_{q^{\prime}}\right)$ such that

$$
\left(\lambda_{0} I-\mathbb{A}_{q^{\prime}}\right) \widetilde{U}=\left(\lambda_{0} I-\mathbb{A}_{q}^{*}\right) W
$$

Let $U \in \mathcal{D}\left(\mathbb{A}_{q}\right)$. Then using the last two formulas, we obtain

$$
\begin{aligned}
\left\langle\left(\lambda_{0} I-\mathbb{A}_{q}\right) U, W\right\rangle_{\mathbb{X}^{q}, \mathbb{X} q^{\prime}}=\left\langle U,\left(\lambda_{0} I-\mathbb{A}_{q}^{*}\right) W\right\rangle_{\mathbb{X} q, \mathbb{X} q^{\prime}}=\left\langle U,\left(\lambda_{0} I-\mathbb{A}_{q^{\prime}}\right) \widetilde{U}\right\rangle_{\mathbb{X} q, \mathbb{X}^{q^{\prime}}} \\
=\left\langle\left(\lambda_{0} I-\mathbb{A}_{q}\right) U, \widetilde{U}\right\rangle_{\mathbb{X}^{q}, \mathbb{X} q^{\prime}} .
\end{aligned}
$$

In particular, we have

$$
\left\langle\left(\lambda_{0} I-\mathbb{A}_{q}\right) U, W-\widetilde{U}\right\rangle_{\mathbb{X}^{q}, \mathbb{X}^{q^{\prime}}}=0 \text { for all } U \in \mathcal{D}\left(\mathbb{A}_{q}\right) .
$$

Therefore $W=\widetilde{U}$ and this completes the proof.

The last result in this section provides some information on the resolvent equation associated to $\mathbb{A}_{q}$.

Proposition 5.4. Let $\lambda \in \mathbb{C}$, such that $\lambda \notin(-\infty, 0)$. Then for every $q \in(1, \infty)$ we have

(i) $\operatorname{Ker}\left(\lambda I-\mathbb{A}_{q}\right)=\{0\}$.

(ii) $\overline{\operatorname{Range}\left(\lambda I-\mathbb{A}_{q}\right)}=\mathbb{X}^{q}$.

Proof. Due to Proposition 3.3 it is enough to show that if $(u, \pi, \ell, \omega) \in\left[W^{2, q}(E)\right]^{3} \times \widehat{W}^{1, q}(E) \times$ $\mathbb{C}^{3} \times \mathbb{C}^{3}$ satisfies the system (4.1) with $\left(f, f_{\ell}, f_{\omega}\right)=0$, then $u=\pi=\ell=\omega=0$.

We first consider the case $q=2$. Multiplying, $4.1{ }_{1}$ by $\left.u, 4.1\right]_{4}$ by $\ell$ and 4.1$]_{5}$ by $\omega$, we obtain after integration by parts:

$$
\lambda \int_{E}|u|^{2}+2 \mu \int_{E}|D(u)|^{2}+\lambda m|\ell|^{2}+\lambda\langle\mathcal{J} \omega, \omega\rangle_{\mathbb{C}^{3}}=0 .
$$

If $\operatorname{Im} \lambda \neq 0$, we take the imaginary part of this identity and obtain that $u=\pi=\ell=\omega=0$. If $\operatorname{Im} \lambda=0$, then $\operatorname{Re} \lambda \geqslant 0$, hence using the above identity and the boundary conditions we also obtain $u=\pi=\ell=\omega=0$. 
Let us then consider the case $q>2$ and $\lambda \neq 0$. Let $B_{1}$ and $B_{2}$ be two open balls in $\mathbb{R}^{3}$ such that

$$
\overline{\mathcal{O}} \subset B_{1}, \quad \overline{B_{1}} \subset B_{2},
$$

and let $\varphi_{1}, \varphi_{2} \in C^{\infty}\left(\mathbb{R}^{3}\right)$ be such that $\varphi_{1}(x) \geqslant 0, \varphi_{2}(x) \geqslant 0, \varphi_{1}(x)+\varphi_{2}(x)=1$ for every $x \in \mathbb{R}^{3}$, $\underline{\varphi_{1}}=1$ on $\overline{B_{1}}, \varphi_{1}=0$ on $\mathbb{R}^{3} \backslash B_{2}, \varphi_{2}=1$ on $\mathbb{R}^{3} \backslash B_{2}$ and $\varphi_{2}=0$ on some open neighbourhood of $\overline{B_{1}}$. Then $\varphi_{1} u$ satisfies the following system

$$
\begin{cases}\lambda\left(\varphi_{1} u\right)-\Delta\left(\varphi_{1} u\right)+\nabla\left(\varphi_{1} \pi\right)=-2(\nabla u)\left(\nabla \varphi_{1}\right)-\left(\Delta \varphi_{1}\right) u+\pi \nabla \varphi_{1} & \left(x \in B_{2} \backslash \overline{\mathscr{O}}\right), \\ \operatorname{div}\left(\varphi_{1} u\right)=\left(\nabla \varphi_{1}\right) \cdot u & \left(x \in B_{2} \backslash \overline{\mathscr{O}}\right), \\ \varphi_{1} u=0 & \left(x \in \partial B_{2}\right), \\ \varphi_{1} u=\ell+\omega \times x & (x \in \partial \mathcal{O}), \\ m \lambda \ell+\int_{\partial \mathcal{O}} \sigma\left(\varphi_{1} u, \varphi_{1} \pi\right) \nu \mathrm{d} s=0, & \\ \mathcal{J} \lambda \omega+\int_{\partial \mathcal{O}} x \times \sigma\left(\varphi_{1} u, \pi\right) \nu=0 . & \end{cases}
$$

Note that $-2(\nabla u)\left(\nabla \varphi_{1}\right)-\left(\Delta \varphi_{1}\right) u+\pi \nabla \varphi_{1} \in\left[L^{2}\left(B_{2} \backslash \overline{\mathscr{O}}\right)\right]^{3}$. Therefore, by using Corollary 3.7 we obtain $\left(\varphi_{1} u, \varphi_{1} \pi\right) \in\left[W^{2,2}\left(B_{2} \backslash \overline{\mathscr{O}}\right)\right]^{3} \times W^{1,2}\left(B_{2} \backslash \overline{\mathscr{O}}\right)$. Similarly, $\left(\varphi_{2} u, \varphi_{2} \pi\right)$ satisfies the following system

$$
\begin{cases}\lambda\left(\varphi_{2} u\right)-\Delta\left(\varphi_{2} u\right)+\nabla\left(\varphi_{2} \pi\right)=-2(\nabla u)\left(\nabla \varphi_{2}\right)-\left(\Delta \varphi_{2}\right) u+\pi \nabla \varphi_{2} & \left(x \in \mathbb{R}^{3}\right) \\ \operatorname{div}\left(\varphi_{2} u\right)=\left(\nabla \varphi_{2}\right) \cdot u & \left(x \in \mathbb{R}^{3}\right)\end{cases}
$$

We also have $2(\nabla u)\left(\nabla \varphi_{2}\right)-\left(\Delta \varphi_{2}\right) u+\pi \nabla \varphi_{2} \in\left[L^{2}\left(\mathbb{R}^{3}\right)\right]^{3}$. By standard results on Stokes operator in the whole space, we also get $\left(\varphi_{2} u, \varphi_{2} \pi\right) \in\left[W^{2,2}\left(\mathbb{R}^{3}\right)\right]^{3} \times \widehat{W}^{1,2}\left(\mathbb{R}^{3}\right)$. Combining the above results we obtain $u \in\left[W^{2,2}(E)\right]^{3}$ and $\pi \in \widehat{W}^{1,2}(E)$.

Let us consider the case $1<q<2$ and $\lambda \neq 0$. We use a bootstrap argument here. Let us set $\bar{f}_{i}=$ $-2(\nabla u)\left(\nabla \varphi_{i}\right)-\left(\Delta \varphi_{i}\right) u+\pi \nabla \varphi_{i}$. By Sobolev imbedding theorem we obtain $\bar{f}_{1}, \bar{f}_{2} \in\left[L^{r}\left(B_{2} \backslash \overline{\mathscr{O}}\right)\right]^{3}$, for $r>q$, with $\frac{1}{3}+\frac{1}{r}=\frac{1}{q}$. This implies that $\varphi_{1} u \in\left[W^{2, r}\left(B_{2} \backslash \overline{\mathscr{O}}\right)\right]^{3}$ and $\varphi_{2} u \in\left[W^{2, r}\left(\mathbb{R}^{3}\right)\right]^{3}$, hence $u \in\left[W^{2, r}(E)\right]^{3}$. If $r \geqslant 2$, we are reduced to the previous case. Otherwise, we continue the process until we get $u \in\left[W^{2,2}(E)\right]^{3}$.

Now we consider the case $\lambda=0$. By looking at the identity 5.10 , we just need to show that $\nabla u \in\left[L^{2}(E)\right]^{9}$. By [3, Theorem 2.1], we know that

$$
\|\nabla u\|_{2, E} \leqslant C\left\|D^{2} u\right\|_{6 / 5, E}
$$

Therefore the conclusion follows as soon as we prove that $D^{2} u \in\left[L^{6 / 5}(E)\right]^{27}$. The argument is similar to the case $\lambda \neq 0$. If $q \geqslant 6 / 5$, then it follows by a similar argument as above. Indeed, we have $\bar{f}_{1} \in\left[L^{6 / 5}\left(B_{2} \backslash \overline{\mathscr{O}}\right)\right]^{3}$ and $\bar{f}_{2} \in\left[L^{6 / 5}\left(\mathbb{R}^{3}\right)\right]^{3}$. Then $D^{2}\left(\varphi_{1} u\right) \in\left[L^{6 / 5}\left(B_{2} \backslash \overline{\mathscr{O}}\right)\right]^{27}$ and $D^{2}\left(\varphi_{2} u\right) \in\left[L^{6 / 5}\left(\mathbb{R}^{3}\right)\right]^{27}$. So we consider the case $1<q<6 / 5$. As before, using embedding theorem we first obtain $\bar{f}_{1} \in\left[L^{s}\left(B_{2} \backslash \overline{\mathscr{O}}\right)\right]^{3}$ and $\bar{f}_{2} \in\left[L^{s}\left(\mathbb{R}^{3}\right)\right]^{3}$ for some $s>q$ with $\frac{1}{n}+\frac{1}{s}=\frac{1}{q}$. Consequently, we have $D^{2}\left(\varphi_{1} u\right) \in\left[L^{s}\left(B_{2} \backslash \overline{\mathscr{O}}\right)\right]^{27}$ and $D^{2}\left(\varphi_{2} u\right) \in\left[L^{s}\left(\mathbb{R}^{3}\right)\right]^{27}$. Observe that $s \geqslant 6 / 5$, thus we are reduced to the previous case. 


\section{Analyticity of the Fluid-Structure semigroup}

We begin by stating the main result in this section, which, besides being of independent interest, is an important ingredient in the proof of our main results. In fact, as mentioned earlier, this result improves the existing result of [27, Theorem 2.5, Theorem 2.9].

Theorem 6.1. For every $1<q<\infty$ and $\theta \in\left(\frac{\pi}{2}, \pi\right)$ there exists $M_{q, \theta}>0$ such that the operator $\mathbb{A}_{q}$ satisfies

$$
\left\|\lambda\left(\lambda I-\mathbb{A}_{q}\right)^{-1}\right\|_{\mathcal{L}\left(\mathbb{X}^{q}\right)} \leqslant M_{q, \theta} \quad\left(\lambda \in \Sigma_{\theta}\right) .
$$

Consequently, $\mathbb{A}_{q}$ generates a bounded analytic semigroup $\mathbb{T}^{q}=\left(\mathbb{T}_{t}^{q}\right)_{t \geqslant 0}$ on $\mathbb{X}^{q}$.

The guiding idea in proving the above result is borrowed from Borchers and Sohr 2 and it consists in using a contradiction argument and appropriate cut-off functions, combined with Proposition 3.6 and classical results for the Stokes operator in the whole space.

A first step towards the proof of Theorem 6.1 is the following result, concerning the case $q \in$ $(1,3 / 2)$ :

Proposition 6.2. Let $q \in\left(1, \frac{3}{2}\right)$ and $\theta \in\left(\frac{\pi}{2}, \pi\right)$. Let $(\mathcal{R}(\lambda))$ and $(P(\lambda))$ be the family of the operators introduced in Theorem 4.3. For $\left(f, f_{\ell}, f_{\omega}\right) \in\left[L^{q}(E)\right]^{3} \times \mathbb{C}^{3} \times \mathbb{C}^{3}$, we set

$$
\left[\begin{array}{l}
u \\
\ell \\
\omega
\end{array}\right]=\mathcal{R}(\lambda)\left[\begin{array}{l}
f \\
f_{\ell} \\
f_{\omega}
\end{array}\right], \quad \pi=\mathcal{P}(\lambda)\left[\begin{array}{c}
f \\
f_{\ell} \\
f_{\omega}
\end{array}\right] \quad\left(\lambda \in \Sigma_{\theta}\right)
$$

Then there exists a constant $M_{q, \theta}>0$ such that, for every $\left(f, f_{\ell}, f_{\omega}\right) \in\left[L^{q}(E)\right]^{3} \times \mathbb{C}^{3} \times \mathbb{C}^{3}$ and for every $\lambda \in \Sigma_{\theta}$,

$$
|\lambda|\left(\|u\|_{q, E}+|\ell|+|\omega|\right)+\left\|D^{2} u\right\|_{q, E}+\|\nabla \pi\|_{q, E}+|\ell|+|\omega| \leqslant M_{q, \theta}\left(\|f\|_{q, E}+\left|f_{\ell}\right|+\left|f_{\omega}\right|\right) .
$$

Proof. First remark that Proposition 5.1 easily implies 6.3 for $\lambda \in \Sigma_{\theta}$ with $|\lambda| \geqslant \gamma$. We thus focus on the proof of the estimate (6.3) for $\lambda \in \Sigma_{\theta}$ with $|\lambda| \leqslant \gamma$. Assume that (6.3) is false for some $q \in\left(1, \frac{3}{2}\right)$ for $\lambda \in \Sigma_{\theta}$ with $|\lambda| \leqslant \gamma$. Then there exists a sequence of complex numbers $\left(\lambda_{n}\right)_{n \in \mathbb{N}}$, together with a sequence $\left(u_{n}, \ell_{n}, \omega_{n}\right)$ in $\mathbb{X}^{q} \cap\left(\left[W^{2, q}(E)\right]^{3} \cap \times \mathbb{C}^{3} \times \mathbb{C}^{3}\right)$ and $\left(\pi_{n}\right)$ in $\widehat{W}^{1, q}(E)$ such that

$$
\begin{gathered}
0<\left|\lambda_{n}\right| \leqslant \gamma, \quad\left|\arg \lambda_{n}\right| \leqslant \theta \quad(n \in \mathbb{N}), \\
\left|\lambda_{n}\right|\left(\left\|u_{n}\right\|_{q, E}+\left|\ell_{n}\right|+\left|\omega_{n}\right|\right)+\left\|D^{2} u_{n}\right\|_{q, E}+\left\|\nabla \pi_{n}\right\|_{q, E}+\left|\ell_{n}\right|+\left|\omega_{n}\right|=1 \quad(n \in \mathbb{N}), \\
\left\|\lambda_{n} u_{n}-\mu \Delta u_{n}+\nabla \pi_{n}\right\|_{q, E} \rightarrow 0, \quad \text { as } n \rightarrow \infty, \\
m \lambda_{n} \ell_{n}+\int_{\partial \mathcal{O}} \sigma\left(u_{n}, \pi_{n}\right) \nu \mathrm{d} s \rightarrow 0, \quad \text { as } n \rightarrow \infty, \\
\mathcal{J} \lambda_{n} \omega_{n}+\int_{\partial \mathcal{O}} x \times \sigma\left(u_{n}, \pi_{n}\right) \nu \mathrm{d} s \rightarrow 0, \quad \text { as } n \rightarrow \infty .
\end{gathered}
$$

To obtain the desired contradiction we proceed, following [2], in several steps.

Step 1: Localization.

Let $B_{1}$ and $B_{2}$ be two open balls in $\mathbb{R}^{3}$ such that

$$
\overline{\mathcal{O}} \subset B_{1}, \quad \overline{B_{1}} \subset B_{2},
$$


and let $\varphi_{1}, \varphi_{2} \in C^{\infty}\left(\mathbb{R}^{3}\right)$ be such that $\varphi_{1}(x) \geqslant 0, \varphi_{2}(x) \geqslant 0, \varphi_{1}(x)+\varphi_{2}(x)=1$ for every $x \in \mathbb{R}^{3}$, $\varphi_{1}=1$ on $\overline{B_{1}}, \varphi_{1}=0$ on $\mathbb{R}^{3} \backslash B_{2}, \varphi_{2}=1$ on $\mathbb{R}^{3} \backslash B_{2}$ and $\varphi_{2}=0$ on some open neighbourhood of $\overline{B_{1}}$. After some calculations, we see that for each $n \in \mathbb{N}$ we have

$$
\begin{cases}\lambda_{n}\left(\varphi_{1} u_{n}\right)-\Delta\left(\varphi_{1} u_{n}\right)+\nabla\left(\varphi_{1} \pi_{n}\right)=\varphi_{1}\left(\lambda_{n} u_{n}-\Delta u_{n}+\nabla \pi_{n}\right) & \\ \quad-2\left(\nabla u_{n}\right)\left(\nabla \varphi_{1}\right)-\left(\Delta \varphi_{1}\right) u_{n}+\pi_{n} \nabla \varphi_{1} & \left(x \in B_{2} \backslash \overline{\mathcal{O}}\right), \\ \operatorname{div}\left(\varphi_{1} u_{n}\right)=\left(\nabla \varphi_{1}\right) \cdot u_{n} & \left(x \in B_{2} \backslash \overline{\mathscr{O}}\right), \\ \varphi_{1} u_{n}=0 & \left(x \in \partial B_{2}\right), \\ \varphi_{1} u_{n}=\ell_{n}+\omega_{n} \times x & (x \in \partial \mathcal{O}), \\ m \lambda_{n} \ell_{n}+\int_{\partial \mathcal{O}} \sigma\left(\varphi_{1} u_{n}, \varphi_{1} \pi_{n}\right) \nu \mathrm{d} s=m \lambda_{n} \ell_{n}+\int_{\partial \mathcal{O}} \sigma\left(u_{n}, \pi_{n}\right) \nu, & \\ \mathcal{J} \lambda_{n} \omega_{n}+\int_{\partial \mathcal{O}} x \times \sigma\left(\varphi_{1} u_{n}, \pi_{n}\right) \nu=\mathcal{J} \lambda_{n} \omega_{n}+\int_{\partial \mathcal{O}} x \times \sigma\left(u_{n}, \pi_{n}\right) \nu \mathrm{d} s . & \end{cases}
$$

By applying Corollary 3.7 and using the fact that $\varphi_{1}$ vanishes outside $B_{2}$, it follows that there exists $c>0$ such that for every $n \in \mathbb{N}$ we have

$$
\begin{aligned}
&\left|\lambda_{n}\right|\left(\left\|\varphi_{1} u_{n}\right\|_{q, E}+\left|\ell_{n}\right|+\left|\omega_{n}\right|\right)+\left\|D^{2}\left(\varphi_{1} u_{n}\right)\right\|_{q, E}+\left\|\nabla\left(\varphi_{1} \pi_{n}\right)\right\|_{q, E}+\left|\ell_{n}\right|+\left|\omega_{n}\right| \\
& \leqslant c\left(\left\|\varphi_{1}\left(\lambda_{n} u_{n}-\Delta u_{n}+\nabla \pi_{n}\right)\right\|_{q, E}+\left\|\nabla\left(\nabla \varphi_{1} \cdot u_{n}\right)\right\|_{q, E}+2\left\|\nabla u_{n} \cdot \nabla \varphi_{1}\right\|_{q, E}\right)+c\left(\left\|\left(\Delta \varphi_{1}\right) u_{n}\right\|_{q, E}+\left\|\pi_{n} \nabla \varphi_{1}\right\|_{q, E}\right) \\
&+c\left(\left|m \lambda_{n} \ell_{n}+\int_{\partial \mathcal{O}} \sigma\left(u_{n}, \pi_{n}\right) \nu\right|+\left|\mathcal{J} \lambda_{n} \omega_{n}+\int_{\partial \mathcal{O}} x \times \sigma\left(u_{n}, \pi_{n}\right) \nu \mathrm{d} s\right|\right) .
\end{aligned}
$$

On the other hand, using the fact that $\varphi_{2}=0$ on some open neighbourhood of $\overline{B_{1}}$, for each $n \in \mathbb{N}$ we have:

$$
\left\{\begin{array}{rr}
\lambda_{n}\left(\varphi_{2} u_{n}\right)-\Delta\left(\varphi_{2} u_{n}\right)+\nabla\left(\varphi_{2} \pi_{n}\right)=\varphi_{2}\left(\lambda_{n} u_{n}-\Delta u_{n}+\nabla \pi_{n}\right) & \\
-2\left(\nabla u_{n}\right)\left(\nabla \varphi_{2}\right)-\left(\Delta \varphi_{2}\right) u_{n}+\pi_{n} \nabla \varphi_{2} & \left(x \in \mathbb{R}^{3}\right), \\
\operatorname{div}\left(\varphi_{2} u_{n}\right)=\left(\nabla \varphi_{2}\right) \cdot u_{n} & \left(x \in \mathbb{R}^{3}\right) .
\end{array}\right.
$$

Using classical results for the Stokes operator in $\mathbb{R}^{3}$ (see, for instance, McCracken 20]), it follows that, for every $n \in \mathbb{N}$ we have

$$
\begin{aligned}
&\left|\lambda_{n}\right|\left\|\varphi_{2} u_{n}\right\|_{q, E}+\left\|D^{2}\left(\varphi_{2} u_{n}\right)\right\|_{q, E}+\left\|\nabla\left(\varphi_{2} \pi_{n}\right)\right\|_{q, E} \\
& \leqslant c\left(\left\|\varphi_{2}\left(\lambda_{n} u_{n}-\Delta u_{n}+\nabla \pi_{n}\right)\right\|_{q, E}+\left\|\nabla\left(\nabla \varphi_{2} \cdot u_{n}\right)\right\|_{q, E}+2\left\|\nabla u_{n} \cdot \nabla \varphi_{2}\right\|_{q, E}\right) \\
& \quad+c\left(\left\|\left(\Delta \varphi_{2}\right) u_{n}\right\|_{q, E}+\left\|\pi_{n} \nabla \varphi_{2}\right\|_{q, E}\right) .
\end{aligned}
$$

By combining $(6.10)$ and 6.12 it follows that for every $n \in \mathbb{N}$ we have

$$
\begin{aligned}
& \left|\lambda_{n}\right|\left(\left\|u_{n}\right\|_{q, E}+\left|\ell_{n}\right|+\left|\omega_{n}\right|\right)+\left\|D^{2} u_{n}\right\|_{q, E}+\left\|\nabla \pi_{n}\right\|_{q, E}+\left|\ell_{n}\right|+\left|\omega_{n}\right| \\
& \leqslant\left|\lambda_{n}\right|\left(\left\|\varphi_{1} u_{n}\right\|_{q, E}+\left\|\varphi_{2} u_{n}\right\|_{q, E}+\left|\ell_{n}\right|+\left|\omega_{n}\right|\right)+\left\|D^{2}\left(\varphi_{1} u_{n}\right)\right\|_{q, E}+\left\|D^{2}\left(\varphi_{2} u_{n}\right)\right\|_{q, E} \\
& +\left\|\nabla\left(\pi_{n} \nabla \varphi_{1}\right)\right\|_{q, E}+\left\|\nabla\left(\pi_{n} \nabla \varphi_{2}\right)\right\|_{q, E} \leqslant c\left\|\lambda_{n} u_{n}-\Delta u_{n}+\nabla \pi_{n}\right\|_{q, E} \\
& +c\left(\left|m \lambda_{n} \ell_{n}+\int_{\partial \mathcal{O}} \sigma\left(u_{n}, \pi_{n}\right) \nu\right|+\left|\mathcal{J} \lambda_{n} \omega_{n}+\int_{\partial \mathcal{O}} x \times \sigma\left(u_{n}, \pi_{n}\right) \nu \mathrm{d} s\right|\right)+W\left(u_{n}, \nabla u_{n}, \pi_{n}\right),
\end{aligned}
$$


where

$$
\begin{aligned}
& W\left(u_{n}, \nabla u_{n}, \pi_{n}\right)=c \sum_{j=1}^{2}\left(\left\|\nabla\left(\nabla \varphi_{j} \cdot u_{n}\right)\right\|_{q, E}+2\left\|\nabla u_{n} \cdot \nabla \varphi_{j}\right\|_{q, E}\right) \\
&+c \sum_{j=1}^{2}\left(\left\|\left(\Delta \varphi_{j}\right) u_{n}\right\|_{q, E}+\left\|\pi_{n} \nabla \varphi_{j}\right\|_{q, E}\right) \quad(n \in \mathbb{N}) .
\end{aligned}
$$

Step 2. Passage to the limit.

Let $r, s>1$ be defined by

$$
\frac{1}{3}+\frac{1}{s}=\frac{1}{q} \quad \text { and } \quad \frac{1}{3}+\frac{1}{r}=\frac{1}{s}
$$

so that

$$
\frac{2}{3}+\frac{1}{r}=\frac{1}{q} \quad \text { and } 1<s<3
$$

By Theorem 2.1 and Lemma 3.1 in Crispo and Maremonti [3] and 6.5, we have

$$
\begin{aligned}
& \left\|u_{n}\right\|_{r, E} \leqslant C\left\|\nabla u_{n}\right\|_{s, E} \leqslant C\left\|D^{2} u_{n}\right\|_{q, E} \leqslant C, \\
& \left\|\pi_{n}\right\|_{s, E} \leqslant C\left\|\nabla \pi_{n}\right\|_{q, E} \leqslant C .
\end{aligned}
$$

Thus, there exist a subsequence, still denoted by $\left(u_{n}\right),\left(\pi_{n}\right),\left(\ell_{n}\right),\left(\omega_{n}\right)$ and $u \in\left[L^{r}(E)\right]^{3}, \pi \in L^{s}(E)$, $(\ell, \omega) \in \mathbb{C}^{3} \times \mathbb{C}^{3}$ and $\lambda \in \overline{\Sigma_{\theta}}$ such that

$$
u_{n} \rightarrow_{\left[L^{r}(E)\right]^{3}} u, \quad \pi_{n} \rightarrow_{L^{s}(E)} \pi, \quad \ell_{n} \rightarrow \ell, \quad \omega_{n} \rightarrow \omega, \quad \lambda_{n} \rightarrow \lambda, \quad \text { as } n \rightarrow \infty,
$$

where $\rightarrow_{X}$ stands for the weak convergence in a Banach space $X$. Let us set

$$
U_{n}=u_{n} \mathbb{1}_{E}+\left(\ell_{n}+\omega_{n} \times x\right) \mathbb{1}_{\mathcal{O}} \quad(n \in \mathbb{N}), \quad U=u \mathbb{1}_{E}+(\ell+\omega \times x) \mathbb{1}_{\mathcal{O}} .
$$

Then $U_{n} \in \mathbb{X}^{r}$ and the sequence $\left(U_{n}\right)$ weakly converges to $U$ in $\mathbb{X}^{r}$. According to $6.6-6.8$ and by the definition of the operator $\mathbb{A}_{q}$, we have that

$$
U_{n} \in \mathcal{D}\left(\mathbb{A}_{q}\right) \text { for all } n \in \mathbb{N}, \quad \text { and }\left(\lambda_{n}-\mathbb{A}_{q}\right) U_{n} \rightarrow_{\mathbb{X}^{q}(E)} 0 \text { as } n \rightarrow \infty .
$$

Let $W \in \mathcal{D}\left(\mathbb{A}_{q^{\prime}}\right) \cap \mathcal{D}\left(\mathbb{A}_{r^{\prime}}\right)$. By Proposition 5.3 .

$$
0=\lim _{n \rightarrow \infty}\left\langle\left(\lambda_{n} I-\mathbb{A}_{q}\right) U_{n}, W\right\rangle_{\mathbb{X}^{q}, \mathbb{X}^{q^{\prime}}}=\left\langle U,\left(\lambda I-\mathbb{A}_{r^{\prime}}\right) W\right\rangle_{\mathbb{X}^{r}, \mathbb{X}^{r^{\prime}}}
$$

Since the set $\left\{\left(\lambda I-\mathbb{A}_{r^{\prime}}\right) W \mid W \in \mathcal{D}\left(\mathbb{A}_{q^{\prime}}\right) \cap \mathcal{D}\left(\mathbb{A}_{r^{\prime}}\right)\right\} \subseteq \mathbb{X}^{q^{\prime}} \cap \mathbb{X}^{r^{\prime}}$ is dense in $\mathbb{X}^{r^{\prime}}$ (see Proposition 5.4, the last formula implies that $U=0$. Consequently, using 6.6.,

$$
\lambda_{n} u_{n} \rightarrow_{\left[L^{q}(E)\right]^{3}} 0, \quad \Delta u_{n} \rightarrow_{\left[L^{q}(E)\right]^{3}} 0, \quad \nabla \pi_{n} \rightarrow_{\left[L^{q}(E)\right]^{3}} 0, \quad \text { as } n \rightarrow \infty .
$$

Next using the fact that $\sup _{n}\left\|\pi_{n}\right\|_{L^{s}(\Omega)}<\infty$ (see 6.17)) we deduce that $\pi=0$.

Now we consider the expression $W\left(u_{n}, \nabla u_{n}, \pi_{n}\right)$ defined in 6.14. We claim that

$$
\lim _{n \rightarrow \infty} W\left(u_{n}, \nabla u_{n}, \pi_{n}\right)=0 .
$$

To shorten the proof, since all the terms in $W\left(u_{n}, \nabla u_{n}, \pi_{n}\right)$ are the same as in [2], we consider only one term of $W\left(u_{n}, \nabla u_{n}, \pi_{n}\right)$, say $f_{j, n}=\nabla\left(\nabla \varphi_{j} \cdot u_{n}\right)$ for $j \in\{1,2\}$, since the other terms can be estimated in a similar manner. Note that, $f_{j, n} \in\left[W_{0}^{1, q}\left(B_{2} \backslash \bar{B}_{1}\right)\right]^{3}$ for every $n \in \mathbb{N}$ and using 
6.16), 6.18 and the fact that $u=0$ we also have $\left(f_{j, n}\right)$ converges weakly to 0 in $\left[L^{q}\left(B_{2} \backslash \bar{B}_{1}\right)\right]^{3}$. Moreover, using (6.17)

$$
\begin{aligned}
\left\|f_{j, n}\right\|_{1, q, B_{2} \backslash \bar{B}_{1}} & \leqslant C\left(\left\|u_{n}\right\|_{q, B_{2} \backslash \bar{B}_{1}}+\left\|\nabla u_{n}\right\|_{q, B_{2} \backslash \bar{B}_{1}}\right) \\
& \leqslant C\left(\left\|u_{n}\right\|_{r, B_{2} \backslash \bar{B}_{1}}+\left\|\nabla u_{n}\right\|_{s, B_{2} \backslash \bar{B}_{1}}\right) \quad(\text { since } r, s>q) \\
& \leqslant C\left(\left\|u_{n}\right\|_{r, E}+\left\|\nabla u_{n}\right\|_{s, E}\right) \leqslant C .
\end{aligned}
$$

Thus, $f_{j, n}$ converges strongly to 0 in $\left[L^{q}\left(B_{2} \backslash \bar{B}_{1}\right)\right]^{3}$ as $n \rightarrow \infty$. Consequently, we obtain (6.20). This, together with (6.5), contradicts the estimate (6.13), which ends the proof.

We are now in position to prove the main result in this section.

Proof of Theorem 6.1. We first note that from Proposition 3.3 , Theorem 4.3 and Proposition 6.2 . we obtain 6.1 for $1<q<\frac{3}{2}$. In the case $\frac{3}{2} \leqslant q \leqslant 2$ we take $q_{0} \in\left(1, \frac{3}{2}\right)$. We define $0 \leqslant s \leqslant 1$ by

$$
\frac{1}{q}=\frac{s}{q_{0}}+\frac{1-s}{2}
$$

Since 6.1 holds for $q_{0}$, there exists a constant $M_{\theta, q_{0}}>0$ such that

$$
\left\|\lambda\left(\lambda I-\mathbb{A}_{q_{0}}\right)^{-1}\right\|_{\mathcal{L}\left(\mathbb{X}^{q_{0}}\right)} \leqslant M_{\theta, q_{0}} \quad\left(\lambda \in \Sigma_{\theta}\right) .
$$

On the other hand, $\mathbb{A}_{2}$ is a self-adjoint operator on $\mathbb{X}^{2}$ (see [23]). Therefore, we also have

$$
\left\|\lambda\left(\lambda I-\mathbb{A}_{2}\right)^{-1}\right\|_{\mathcal{L}\left(\mathbb{X}^{2}\right)} \leqslant M_{\theta, 2} \quad\left(\lambda \in \Sigma_{\theta}\right),
$$

for some $M_{\theta, 2}$ depending only on $\theta$. Then by Riesz-Thorin interpolation theorem (see for instance [25, Theorem 1, Section 1.18.7]), we obtain

$$
\left\|\lambda\left(\lambda I-\mathbb{A}_{q}\right)^{-1}\right\|_{\mathcal{L}\left(\mathbb{X}^{q}\right)} \leqslant M_{\theta, q_{0}}^{s} M_{\theta, 2}^{1-s} \quad\left(\lambda \in \Sigma_{\theta}\right) .
$$

This ends the proof of 6.1 for $\frac{3}{2} \leqslant q \leqslant 2$.

In the case $2<q<\infty$, we take $1<q^{\prime} \leqslant 2$ such that $\frac{1}{q}+\frac{1}{q^{\prime}}=1$. By Proposition 5.3 , we have $\lambda\left(\lambda I-\mathbb{A}_{q}\right)^{-1}=\left[\lambda\left(\lambda I-\mathbb{A}_{q^{\prime}}\right)^{-1}\right]^{*}$, so that

$$
\left\|\lambda\left(\lambda I-\mathbb{A}_{q}\right)^{-1}\right\|_{\mathcal{L}\left(\mathbb{X}^{q}\right)}=\left\|\lambda\left(\lambda I-\mathbb{A}_{q^{\prime}}\right)^{-1}\right\|_{\mathcal{L}\left(\mathbb{X}^{q^{\prime}}\right)} \quad\left(\lambda \in \Sigma_{\theta}\right) .
$$

We have already seen that (6.1) holds for $1<q \leqslant 2$. Thus from the above identity we infer that, (6.1) holds for any $2<q<\infty$, which ends the proof.

We end this section with the result below, whose proof can be easily obtained by combining Theorem 6.1 and the results from Lunardi [17, Chapter 3]:

Corollary 6.3. With the assumptions and notations of Theorem 6.1, for any $\varepsilon>0$ there exists $C_{\varepsilon}>0$, such that

$$
\left\|\mathbb{A}_{q}^{k-1} U\right\|_{\mathbb{X}^{q}} \leqslant \varepsilon\left\|\mathbb{A}_{q}^{k} U\right\|_{\mathbb{X}^{q}}+C_{\varepsilon}\|U\|_{\mathbb{X}^{q}} \quad\left(U \in \mathcal{D}\left(\mathbb{A}_{q}^{k}\right), k \in \mathbb{N}\right) .
$$




\section{Decay estimates for the fluid-structure semigroup}

Based on Theorem 6.1, we consider the fluid-structure semigroup which is, for each $q \in(1, \infty)$, the bounded analytic semigroup $\mathbb{T}^{q}$ introduced in Theorem 6.1. Our main result in this section is:

Theorem 7.1. (i) Let $1<q<\infty$. Let $R_{0}>0$ be such that $\overline{\mathcal{O}} \subset B_{R_{0}}$. Then for any $R>R_{0}$, there exists a constant $C>0$, depending on $q$ and $R$, such that

$$
\left\|\mathbb{T}_{t}^{q} U\right\|_{q, B_{R}} \leqslant C t^{-\frac{3}{2 q}}\|U\|_{\mathbb{X}^{q}} \quad\left(t>1, U \in \mathbb{X}^{q}\right) .
$$

(ii) Let $1<q \leqslant r<\infty$ and $\sigma=\frac{3}{2}\left(\frac{1}{q}-\frac{1}{r}\right)$. Then there exists a constant $C>0$, depending on $q$ and $r$, such that

$$
\left\|\mathbb{T}_{t}^{q} U\right\|_{\mathbb{X}^{r}} \leqslant C t^{-\sigma}\|U\|_{\mathbb{X}^{q}} \quad\left(t>0, U \in \mathbb{X}^{q}\right) .
$$

(iii) Let $1<q \leqslant r \leqslant 3$. Then there exists a constant $C>0$, depending on $q$ and $r$, such that

$$
\left\|\nabla \mathbb{T}_{t}^{q} U\right\|_{r, E} \leqslant C t^{-\sigma-1 / 2}\|U\|_{\mathbb{X}^{q}} \quad\left(t>0, U \in \mathbb{X}^{q}\right) .
$$

(iv) Estimate (7.2) also holds for $1<q<\infty$ and $r=\infty$.

Let us emphasize that Theorem 7.1 holds for the linearized fluid-structure equations for bodies $\mathcal{O}$ of arbitrary shapes. It seems thus likely that these properties can be used to derive the wellposedness for solids of arbitrary shape, see the discussion in Section 9 below.

Let us also mention that, Maremonti and Solonnikov in 19] proved that, while considering Stokes equation in the exterior domain, the decay estimate 7.3 is sharp. It is then expected that same holds for the fluid-structure operator also.

Our methodology to prove the above result is inspired by 13 and it consists in using the resolvent estimates developed in Section 4-Section 6. However, applying the strategy proposed in 13 requires several adaptations which are described below.

To start with, we state the following regularity result of the projection operator $\mathbb{P}_{q}$

Proposition 7.2. Let $k \in \mathbb{N}$. Assume that $1<r \leqslant q<\infty$. Let $u \in\left[L^{q}\left(\mathbb{R}^{3}\right)\right]^{3}$ be such that $\operatorname{div} u=0$ in $\mathcal{D}^{\prime}\left(\mathbb{R}^{3}\right)$ and $\partial^{\alpha} u \in\left[L^{r}(E)\right]^{3}$ for every multi-index $\alpha \in \mathbb{Z}_{+}^{3}$ with $|\alpha|=k$. Then $\partial^{\alpha}\left(\mathbb{P}_{q} u\right) \in\left[L^{r}(E)\right]^{3}$ for every multi-index $\alpha \in \mathbb{Z}_{+}^{3}$ with $|\alpha|=k$. Moreover, there exists a constant $C$ independent of the choice of $u$ with the above properties, such that

$$
\sum_{|\alpha|=k}\left\|\partial^{\alpha} \mathbb{P}_{q} u\right\|_{r, E} \leqslant C\left(\sum_{|\alpha|=k}\left\|\partial^{\alpha} u\right\|_{r, E}+\|u\|_{q}\right) .
$$

Proof. Let $v=\mathbb{P}_{q} u$. Then

$$
v(x)=\ell_{v}+\omega_{v} \times x \quad(x \in \mathcal{O}),
$$

where

$$
\ell_{v}=\frac{1}{m} \int_{\mathcal{O}} v \mathrm{~d} x, \quad \omega_{v}=-\frac{1}{\mathcal{J}} \int_{\mathcal{O}} v \times x \mathrm{~d} x .
$$

Moreover, there exists a positive constant $C$, depending only on $q$ and on $\mathcal{O}$, such that (see for instance [27, Proof of Theorem 2.2, Eq. (3.14)])

$$
\left|\ell_{v}\right|+\left|\omega_{v}\right| \leqslant C\|u\|_{q} .
$$

Since $\operatorname{div} u=0$, we have that $w_{1}$ from the decomposition (3.4) of $u$ vanishes and, according to 27 , Proof of Theorem 2.2, Eq. (3.15)], $w_{2}$ from the same decomposition satisfies $w_{2}=\nabla \pi_{2}$, with $\pi_{2}$ satisfying

$$
\Delta \pi_{2}=0 \text { in } E, \quad \frac{\partial \pi_{2}}{\partial \nu}=u \cdot \nu-\left(\ell_{v}+\omega_{v} \times x\right) \cdot \nu \text { on } \partial \mathcal{O}
$$

Then estimate (7.4) follows from (7.7) and from Giga and Sohr [8, proof of Lemma 2.3]. 
We next provide two results characterising the graph norm of $\mathbb{A}_{q}^{m}$ in terms of Sobolev spaces.

Proposition 7.3. Let $1<q<\infty$.

(i) Assume that $U \in \mathcal{D}\left(\mathbb{A}_{q}\right)$ and $\mathbb{A}_{q} U_{\mid E} \in\left[W^{m, q}(E)\right]^{3}$ for some $m \in \mathbb{Z}_{+}$. Then $U_{\mid E} \in\left[W^{m+2, q}(E)\right]^{3}$ and there exists a constant $C_{m}>0$ such that

$$
\|U\|_{m+2, q, E} \leqslant C_{m}\left(\left\|\mathbb{A}_{q} U\right\|_{m, q, E}+\|U\|_{\mathbb{X}^{q}}\right) .
$$

(ii) For every $m \in \mathbb{N}$, if $U \in \mathcal{D}\left(\mathbb{A}_{q}^{m}\right)$, then $U_{\mid E} \in W^{2 m, q}(E)$ and

$$
\|U\|_{2 m, q, E} \leqslant C\left(\left\|\mathbb{A}_{q}^{m} U\right\|_{\mathbb{X}^{q}}+\|U\|_{\mathbb{X}^{q}}\right) \quad\left(U \in \mathcal{D}\left(\mathbb{A}_{q}^{m}\right)\right) .
$$

Proof. Let us set $\mathbb{A}_{q} U=-F$, so that $F_{\mid E} \in\left[W^{m, q}(E)\right]^{3}$. Moreover, we denote

$$
u=U_{\mid E}, \quad \ell=\frac{1}{m} \int_{\mathcal{O}} U \mathrm{~d} x, \quad \omega=-\mathcal{J}^{-1} \int_{\mathcal{O}} U \times x \mathrm{~d} x .
$$

Then according to Proposition 5.2 there exists $\pi \in \widehat{W}^{1, q}(E)$ such that $u, \pi, \ell$ and $\omega$ satisfy

$$
\begin{cases}-\mu \Delta u+\nabla \pi=F & (x \in E), \\ \operatorname{div} u=0 & (x \in E), \\ u=\ell+\omega \times x & (x \in \partial \mathcal{O}) .\end{cases}
$$

Let $\left[\begin{array}{l}w_{1} \\ \eta_{1}\end{array}\right]=D_{1}\left[\begin{array}{l}\ell \\ \omega\end{array}\right]$, where $D_{1}$ is the Dirichlet map introduced in Proposition 4.1 According to Proposition 4.1, for every $k \in \mathbb{N}$ there exists positive constants $C_{1, k}, C_{2, k}$ such that

$$
\left\|w_{1}\right\|_{k+1, q, E}+\left\|\pi_{1}\right\|_{\widehat{W}^{k, q}(E)} \leqslant C_{1, k}(|\ell|+|\omega|) \leqslant C_{2, k}\|U\|_{\mathbb{X}^{q}}
$$

We denote $\widetilde{u}=u-w_{1}$ and $\widetilde{\pi}=\pi-\eta_{1}$. Then $\widetilde{u}$ and $\widetilde{\pi}$ satisfy

$$
\begin{cases}-\mu \Delta \widetilde{u}+\nabla \widetilde{\pi}=F+w_{1} & (x \in E), \\ \operatorname{div} \widetilde{u}=0 & (x \in E), \\ \widetilde{u}=0 & (x \in \partial \mathcal{O}) .\end{cases}
$$

According to [13, Proposition 2.7(i)], for every $m \in \mathbb{N}$ there exists a positive constant $C_{3, m}$ such that

$$
\|\widetilde{u}\|_{m+2, q, E} \leqslant C_{3, m}\left(\|F\|_{m, q, E}+\left\|w_{1}\right\|_{m, q, E}+\|\widetilde{u}\|_{q, E}\right) .
$$

The above estimate together with 7.11 implies the estimate 7.9 .

To prove 7.10 , we use an induction argument. We first note that 7.10 is true for $m=1$, since it is nothing else but the estimate 7.9 for $m=0$. Let us assume that 7.10 is true for some $m \in \mathbb{N}$ and $U \in \mathcal{D}\left(\mathbb{A}_{q}^{m+1}\right)$. Then by 7.9 and induction hypothesis, there exists a positive constant $C_{m}>0$ such that

$$
\|U\|_{2 m+2, q, E} \leqslant C_{m}\left(\left\|\mathbb{A}_{q} U\right\|_{2 m, q, E}+\|U\|_{\mathbb{X}^{q}}\right) \leqslant C\left(\left\|\mathbb{A}_{q}^{m+1} U\right\|_{\mathbb{X}^{q}}+\left\|\mathbb{A}_{q} U\right\|_{\mathbb{X}^{q}}+\|U\|_{\mathbb{X}^{q}}\right) .
$$

Then the assertion 7.10 holds for $m$ replaced by $m+1$ by applying Corollary 6.3 repeatedly and 7.12. This completes the proof of the proposition. 
Proposition 7.4. Let $q \in(1, \infty)$. Then:

(i) For any $m \in \mathbb{N}$, there exists a positive constant $C_{m}>0$ such that

$$
\left\|\mathbb{A}_{q}^{m} U\right\|_{\mathbb{X}^{q}} \leqslant C_{m}\left(\|U\|_{2 m, q, E}+\|U\|_{\mathbb{X}^{q}}\right) \quad\left(U \in \mathcal{D}\left(\mathbb{A}_{q}^{m}\right)\right) .
$$

(ii) Let $\theta \in\left(\frac{\pi}{2}, \pi\right)$ and $m \in \mathbb{N}$. Then there exists a positive constant $C_{m}>0$ such that

$$
\begin{array}{r}
\left\|\left(\lambda I-\mathbb{A}_{q}\right)^{-1} F\right\|_{2 m+2, q, E} \leqslant C_{m}\left(\|F\|_{2 m, q, E}+\|F\|_{\mathbb{X}^{q}}\right), \\
\left(F \in \mathcal{D}\left(\mathbb{A}_{q}^{m}\right), \lambda \in \Sigma_{\theta},|\lambda| \geqslant 1\right) .
\end{array}
$$

Proof. We use an induction argument to prove (7.13). Using Proposition 3.1. (3.7) and (3.8) we first note that the estimate $(7.9)$ is true for $m=1$. Assume that $(7.13)$ holds for some $m \in \mathbb{N}$ and $U \in \mathcal{D}\left(\mathbb{A}_{q}^{m+1}\right)$. By the induction hypothesis, we have

$$
\left\|\mathbb{A}_{q}^{m+1} U\right\|_{\mathbb{X}^{q}} \leqslant C_{m}\left(\left\|\mathbb{A}_{q} U\right\|_{2 m, q, E}+\left\|\mathbb{A}_{q} U\right\|_{\mathbb{X}^{q}}\right), \quad\left(U \in \mathcal{D}\left(\mathbb{A}_{q}^{m+1}\right)\right) .
$$

By applying Proposition 7.2 and Corollary 6.3 , the above estimate implies that

$$
\left\|\mathbb{A}_{q}^{m+1} U\right\|_{\mathbb{X}^{q}} \leqslant C_{m}\left(\|U\|_{2 m+2, q, E}+\|U\|_{\mathbb{X}^{q}}\right) \quad\left(U \in \mathcal{D}\left(\mathbb{A}_{q}^{m+1}\right)\right) .
$$

Thus (7.13) also holds when $m$ is replaced by $m+1$.

Finally (7.14) follows from the facts that

$$
\left\|\lambda\left(\lambda I-\mathbb{A}_{q}\right)^{-1}\right\|_{\mathcal{L}\left(\mathcal{D}\left(\mathbb{A}_{q}^{m}\right)\right)} \leqslant M, \quad\left(\lambda \in \Sigma_{\theta}\right)
$$

together with the estimates 7.10 and 7.13 .

Remark 7.5. Putting together (7.10) and (7.13), it follows that, for every $m \in \mathbb{N}$, the graph norm of $\mathbb{A}_{q}^{m}$ is equivalent to $\|\cdot\|_{2 m, q, E}+\|\cdot\|_{\mathbb{X}^{q}}$. We also note that this equivalence also holds for the bounded domain version of the fluid-structure operator, i.e., $\Omega \subset \mathbb{R}^{3}$ open and bounded, and the operator $\mathbb{A}_{q, \Omega}$ defined in 3.7 . Moreover, elements $\varphi$ of $\mathcal{D}\left(\mathbb{A}_{q}^{m}\right)$ belong to $\left[W^{1, q}\left(\mathbb{R}^{3}\right)\right]^{3} \cap \mathbb{X}^{q}\left(\mathbb{R}^{3}\right)$ and satisfy $\varphi_{\mid E} \in\left[W^{2 m, q}(E)\right]^{3}$.

To state the next results, which yield decay estimates for the fluid-structure semigroup in weighted $L^{p}$ spaces, we remind from Section 2 the notation $L^{q, s}$ for the weighted Lebesgue spaces introduced in 2.3.

Theorem 7.6. Let $1<q<\infty$. Let $s$ and $s^{\prime}$ be real numbers such that $s>3(1-1 / q)$ and $s^{\prime}<-3 / q$. Then there exists a positive constant $C$, depending only on $q, s$ and $s^{\prime}$, such that

$$
\left\|\mathbb{T}_{t}^{q} U\right\|_{\left[L^{q, s^{\prime}}\left(\mathbb{R}^{3}\right)\right]^{3}} \leqslant C(1+t)^{-\frac{3}{2}}\|U\|_{\left[L^{q, s}\left(\mathbb{R}^{3}\right)\right]^{3}} \quad U \in \mathbb{X}^{q} \cap\left[L^{q, s}\left(\mathbb{R}^{3}\right)\right]^{3} .
$$

Proof. We first note that Theorem 4.3 is a complete analogue of Corollary 3.2 in [13], and Theorem 6.1 is the analogue of the main result in 2 . We can thus complete the proof following line by line the proof of Theorem 1.1 in [13].

Remark 7.7. For $U_{0} \in \mathcal{D}\left(\mathbb{A}_{q}\right)$, we denote by

$$
u_{0}=\left.U_{0}\right|_{E}, \quad \ell_{0}=\frac{1}{m} \int_{\mathcal{O}} U_{0} \mathrm{~d} x, \quad \omega_{0}=-\frac{1}{\mathcal{J}} \int_{\mathcal{O}} U_{0} \times x \mathrm{~d} x .
$$


Moreover for every $t \geqslant 0$, we set $U(t)=\mathbb{T}_{t}^{q} U_{0}$ and

$$
u(t)=\left.U(t)\right|_{E}, \quad \ell(t)=\frac{1}{m} \int_{\mathcal{O}} U(t) \mathrm{d} x, \quad \omega(t)=-\frac{1}{\mathcal{J}} \int_{\mathcal{O}} U(t) \times x \mathrm{~d} x .
$$

Then there exists $\pi \in C\left([0, \infty) ; \widehat{W}^{1, q}(E)\right)$ such that $(u, \pi, \ell, \omega)$ satisfies the following system

$$
\begin{cases}\partial_{t} u-\mu \Delta u+\nabla \pi=0, \quad \operatorname{div} u=0 & (t \geqslant 0, x \in E), \\ u=\ell+\omega \times x & (t \geqslant 0, x \in \partial \mathcal{O}), \\ m \dot{\ell}+\int_{\partial \mathcal{O}} \sigma(u, \pi) \nu \mathrm{d} s=0 & (t \geqslant 0), \\ \mathcal{J} \dot{\omega}+\int_{\partial \mathcal{O}} x \times \sigma(u, \pi) \nu \mathrm{d} s=0, & (t \geqslant 0), \\ u(0)=u_{0} & (x \in E), \\ \ell(0)=\ell_{0}, \quad \omega(0)=\omega_{0} . & \end{cases}
$$

Our next result in this section provides $L^{q}-L^{r}$ decay estimates for the fluid-structure semigroup $\mathbb{T}^{q}$ for small time:

Theorem 7.8. Let $1<q \leqslant r<\infty$ and $\sigma=\frac{3}{2}\left(\frac{1}{q}-\frac{1}{r}\right)$. Then for each $\tau \in(0, \infty)$, there exists a constant $C>0$, depending on $\tau, q$ and $r$, such that

$$
\begin{array}{lr}
\left\|\mathbb{T}_{t}^{q} U\right\|_{\mathbb{X}^{r}} \leqslant C t^{-\sigma}\|U\|_{\mathbb{X}^{q}} & \left(t \leqslant \tau, U \in \mathbb{X}^{q}\right), \\
\left\|\nabla \mathbb{T}_{t}^{q} U\right\|_{r, E} \leqslant C t^{-\sigma-\frac{1}{2}}\|U\|_{\mathbb{X}^{q}} & \left(t \leqslant \tau, U \in \mathbb{X}^{q}\right) .
\end{array}
$$

Proof. Let $N=[2 \sigma]$, where [.] denotes the integer part function. Let us assume that $N$ is even. Then by (7.10), there exists a constant $C>0$ depending on $\tau, q$ and $r$, such that

$$
\begin{aligned}
\left\|\mathbb{T}_{t}^{q} U\right\|_{N, q, E}+|\ell(t)| & +|\omega(t)| \leqslant C\left(\left\|\mathbb{A}_{q}^{N / 2} \mathbb{T}_{t}^{q} U\right\|_{\mathbb{X}^{q}}+\left\|\mathbb{T}_{t}^{q} U\right\|_{\mathbb{X}^{q}}\right) \\
& \leqslant C\left(t^{-\frac{N}{2}}\|U\|_{\mathbb{X}^{q}}+\tau^{\frac{N}{2}} t^{-\frac{N}{2}}\|U\|_{\mathbb{X}^{q}}\right) \leqslant C t^{-\frac{N}{2}}\|U\|_{\mathbb{X}^{q}} \quad(t \in(0, \tau]) .
\end{aligned}
$$

In a similar manner, we also obtain

$$
\left\|\mathbb{T}_{t}^{q} U\right\|_{N+2, q, E}+|\ell(t)|+|\omega(t)| \leqslant C t^{-\frac{N+2}{2}}\|U\|_{\mathbb{X}^{q}} \quad(t \in(0, \tau]) .
$$

Thus by Sobolev embedding, interpolation and using (7.22)-(7.23), we obtain

$$
\begin{aligned}
\left\|\mathbb{T}_{t}^{q} U\right\|_{\mathbb{X}^{r}} & \leqslant C\left(\left\|\mathbb{T}_{t}^{q} U\right\|_{r, E}+|\ell(t)|+|\omega(t)|\right) \\
& \leqslant C\left(\left\|\mathbb{T}_{t}^{q} U\right\|_{2 \sigma, q, E}+|\ell(t)|+|\omega(t)|\right) \\
& \leqslant C\left(\left\|\mathbb{T}_{t}^{q} U\right\|_{N+2, q, E}^{2 \frac{2-N}{2}}\left\|\mathbb{T}_{t}^{q} U\right\|_{N, q, E}^{\frac{N+2-2 \sigma}{2}}+|\ell(t)|+|\omega(t)|\right) \leqslant C t^{-\sigma}\|U\|_{\mathbb{X}^{q}}, \quad t \in(0, \tau] .
\end{aligned}
$$

If $N$ is odd then we replace $N$ by $N-1$. This completes the proof of 7.20 . The proof of 7.21) is completely similar, thus omitted here.

The next step towards the proof of Theorem 7.1 is the following result:

Lemma 7.9. With the notations and assumptions of Theorem 7.1. let $d>R_{0}$ and let $m \in \mathbb{N}$. Moreover, denote $E_{d}:=\{x \in E|| x \mid<d\}$. Then 
(i) There exists a constant $C>0$ depending on $d$ and $m$ such that for all $t>0$,

$$
\left\|\mathbb{T}_{t}^{q} U\right\|_{q, B_{d}}+\left\|\mathbb{T}_{t}^{q} U\right\|_{2 m, q, E_{d}} \leqslant C(1+t)^{-\frac{3}{2}}\left(\|U\|_{2 m, q, E_{d}}+\|U\|_{\mathbb{X}^{q}}\right),
$$

for every $U \in \mathcal{D}\left(\mathbb{A}_{q}^{m}\right)$ with $U=0$ for $|x|>d$.

(ii) There exists a constant $C>0$ depending on $d$ and $m$ such that for all $t>0$,

$$
\left\|\partial_{t} \mathbb{T}_{t}^{q} U\right\|_{q, B_{d}}+\left\|\partial_{t} \mathbb{T}_{t}^{q} U\right\|_{2 m, q, E_{d}} \leqslant C(1+t)^{-\frac{5}{2}}\left(\|U\|_{2 m+2, q, E_{d}}+\|U\|_{\mathbb{X}^{q}}\right),
$$

for every $U \in \mathcal{D}\left(\mathbb{A}_{q}^{m+1}\right)$ with $U=0$ for $|x|>d$.

Proof. The proof can be obtained following line by line the proof Lemma 5.2 from Iwashita [13. More precisely, it suffices to use instead of Proposition 2.7 and Lemma 2.8 in [13. our results in Proposition 7.3 and Proposition 7.4 above, respectively, and to replace expansion (3.2) in [13] by 4.15 above.

Proposition 7.10. With the notation and assumptions of Remark 7.7 and Theorem 7.1, let $d>$ $R_{0}+5$ and $m \in \mathbb{N}$. Moreover, assume that $U_{0} \in \operatorname{Ran}\left(\mathbb{T}_{1}^{q}\right)$. Then there exists a positive constant $C$, depending only on $d, m$ and $q$, such that, for every $t \geqslant 0$ we have

$$
\begin{aligned}
& \|u(t, \cdot)\|_{2 m, q, E_{d}}+|\ell(t)|+|\omega(t)| \leqslant C(1+t)^{-\frac{3}{2 q}}\left(\left\|u_{0}\right\|_{[3 / q]+2 m+2, q, E}+\left|\ell_{0}\right|+\left|\omega_{0}\right|\right), \\
& \left\|\partial_{t} u(t, \cdot)\right\|_{2 m, q, E_{d}}+|\dot{\ell}(t)|+|\dot{\omega}(t)| \leqslant C(1+t)^{-\frac{3}{2 q}}\left(\left\|u_{0}\right\|_{[3 / q]+2 m+4, q, E}+\left|\ell_{0}\right|+\left|\omega_{0}\right|\right), \\
& \|\pi(t, \cdot)\|_{2 m+1, q, E_{d}} \leqslant C(1+t)^{-\frac{3}{2 q}}\left(\left\|u_{0}\right\|_{[3 / q]+2 m+4, q, E}+\left|\ell_{0}\right|+\left|\omega_{0}\right|\right),
\end{aligned}
$$

where $[s]$ denotes the integer part of $s \in \mathbb{R}$.

Proof. We follow with minor modifications the steps of the proof of Lemma 5.3 in [13].

Step 1. Since $U_{0} \in \operatorname{Ran}\left(\mathbb{T}_{1}^{q}\right)$, we have $U_{0} \in \mathcal{D}\left(\mathbb{A}_{q}^{k}\right)$ for all $k \in \mathbb{N}$. Let $\widetilde{u}_{0}$ be an extension of $u_{0}$ to $\mathbb{R}^{3}$ such that $\widetilde{u}_{0} \in\left[W^{2 m, q}\left(\mathbb{R}^{3}\right)\right]^{3}$ and $\left\|\widetilde{u}_{0}\right\|_{2 m, q} \leqslant C\left\|U_{0}\right\|_{\mathcal{D}\left(\mathbb{A}_{q}^{m}\right)}$, where $C$ is a constant independent of $U_{0}$. Then $\operatorname{div} \widetilde{u}_{0} \in W_{0}^{2 m-1, q}(\mathcal{O})$ and $\int_{\mathcal{O}} \operatorname{div} \widetilde{u}_{0}=\int_{\partial \mathcal{O}}\left(\ell_{0}+\omega_{0} \times x\right) \cdot \nu \mathrm{d} s=0$. Then by Lemma 2.1 we have that $\mathbb{B}_{\mathcal{O}}\left(\operatorname{div} \widetilde{u}_{0}\right) \in W_{0}^{2 m, q}(\mathcal{O})$. Let us set

$$
\psi=\widetilde{u}_{0}-\mathbb{B}_{\mathcal{O}}\left(\operatorname{div} \widetilde{u}_{0}\right),
$$

where $\mathbb{B}_{\mathcal{O}}\left(\operatorname{div} \widetilde{u}_{0}\right)$ is seen as a function in $\left[W^{2 m, q}\left(\mathbb{R}^{3}\right)\right]^{3}$ after its extension by 0 in $E$. Then $\psi \in$ $\left[W^{2 m, q}\left(\mathbb{R}^{3}\right)\right]^{3}$ has the following properties

$$
\psi(x)=U_{0}(x)=u_{0}(x) \text { for all } x \in \mathbb{E}, \quad \operatorname{div} \psi(x)=0 \text { in } \mathbb{R}^{3}, \quad\|\psi\|_{2 m, q} \leqslant C\left\|U_{0}\right\|_{\mathcal{D}\left(\mathbb{A}_{q}^{m}\right)} .
$$

Step 2. We consider the following Stokes system in $\mathbb{R}^{3}$

$$
\begin{cases}\partial_{t} v_{0}(t, x)-\mu \Delta v_{0}(t, x)=0, \operatorname{div} v_{0}(t, x)=0 & \left(t \geqslant 0, x \in \mathbb{R}^{3}\right) \\ v_{0}(0, x)=\psi(x) & \left(x \in \mathbb{R}^{3}\right)\end{cases}
$$

Let $q$ and $r$ be such that $1<q \leqslant r \leqslant \infty$ and define $\sigma=\frac{3}{2}\left(\frac{1}{q}-\frac{1}{r}\right)$. According to classical estimates (see, for instance, [13, Lemma 5.1]) for the heat kernel, for every $m \in \mathbb{Z}_{+}$, there exists a constant 
$C_{m}>0$, depending on $q$ and $r$, with

$$
\begin{array}{ll}
\left\|\nabla^{m} v_{0}(t, \cdot)\right\|_{r} \leqslant C_{m} t^{-\sigma-\frac{m}{2}}\|\psi\|_{q} & (t>0), \\
\left\|\nabla^{m} v_{0}(t, \cdot)\right\|_{r} \leqslant C_{m}(1+t)^{-\sigma-\frac{m}{2}}\|\psi\|_{[2 \sigma]+m+1, q} & (t \geqslant 0), \\
\left\|\nabla^{m} \partial_{t} v_{0}(t, \cdot)\right\|_{r} \leqslant C_{m} t^{-\sigma-1-m / 2}\|\psi\|_{q} & (t>0), \\
\left\|\nabla^{m} \partial_{t} v_{0}(t, \cdot)\right\|_{r} \leqslant C_{m}(1+t)^{-\sigma-1-\frac{m}{2}}\|\psi\|_{[2 \sigma]+m+3, q} & (t \geqslant 0) .
\end{array}
$$

Let $\varphi \in C_{0}^{\infty}\left(\mathbb{R}^{3}\right)$ be such that $\varphi(x)=1$ for $|x| \leqslant d-2$ and $\varphi(x)=0$ for $|x|>d-1$. Denote $\Omega_{d}=\left\{x \in \mathbb{R}^{3}|d-2 \leqslant| x \mid \leqslant d-1\right\}$ and let $\mathbb{B}_{d}: \mathcal{D}\left(\Omega_{d}\right) \rightarrow\left[\mathcal{D}\left(\Omega_{d}\right)\right]^{3}$ be the Bogovskii operator such that $\operatorname{div}\left(\mathbb{B}_{d} h\right)=h$ if $\int_{\Omega_{d}} h=0$. We define

$$
v_{1}(t, \cdot)=\mathbb{B}_{d}\left(-\nabla \varphi \cdot v_{0}(t, \cdot)\right) \quad(t \geqslant 0) .
$$

By applying (7.32) and (7.34, it follows that there exists a constant $C_{m}>0$, depending on $q$, such that

$$
\begin{aligned}
& \left\|v_{1}(t, \cdot)\right\|_{m, q, E} \leqslant C_{m}\left\|\nabla \varphi \cdot v_{0}(t, \cdot)\right\|_{m-1, q, E} \leqslant C_{m}(1+t)^{-3 / 2 q}\|\psi\|_{[3 / q]+m, q} \quad(t \geqslant 0), \quad(7.36) \\
& \left\|\partial_{t} v_{1}(t, \cdot)\right\|_{m, q, E} \leqslant C_{m}\left\|\nabla \varphi \cdot \partial_{t} v_{0}(t, \cdot)\right\|_{m-1, q, E} \leqslant C_{m}(1+t)^{-3 / 2 q}\|\psi\|_{[3 / q]+m+2, q} \quad(t \geqslant 0) .
\end{aligned}
$$

Step 3. We now set

$$
v_{2}(t, x)=u(t, x)-(1-\varphi) v_{0}(t, x)+v_{1}(t, x) \quad(t \geqslant 0, x \in E) .
$$

Then $v_{2}, \pi, \ell$ and $\omega$ satisfy

$$
\begin{cases}\partial_{t} v_{2}-\mu \Delta v_{2}+\nabla \pi=f, \quad \operatorname{div} v_{2}=0 & (t>0, x \in E), \\ v_{2}=\ell+\omega \times x & (t>0, x \in \partial \mathcal{O}), \\ m \dot{\ell}+\int_{\partial \mathcal{O}} \sigma\left(v_{2}, \pi\right) \nu \mathrm{d} s=0 & (t>0), \\ \mathcal{J} \dot{\omega}+\int_{\partial \mathcal{O}} x \times \sigma\left(v_{2}, \pi\right) \nu \mathrm{d} s=0 & (t>0), \\ v_{2}(0, x)=\zeta(x) & (x \in E), \\ \ell(0)=\ell_{0}, \quad \omega(0)=\omega_{0}, & \end{cases}
$$

where

$$
\begin{gathered}
f=-2(\nabla \varphi \cdot \nabla) v_{0}-\mu(\Delta \varphi) v_{0}+\partial_{t} v_{1}-\mu \Delta v_{1} \\
\zeta(x)=\varphi(x) \psi(x)+v_{1}(0, x) \quad(x \in E) .
\end{gathered}
$$

Moreover, we have

$$
\begin{array}{cc}
\operatorname{div} f(t, x)=0, \quad \operatorname{div} \zeta(x)=0 & (t>0, x \in E), \\
\operatorname{supp} f(t, \cdot) \subset\{d-2 \leqslant|x| \leqslant d-1\} & (t>0) .
\end{array}
$$

Denote

$$
V_{20}=\zeta \mathbb{1}_{E}+\left(\ell_{0}+\omega_{0} \times x\right) \mathbb{1}_{\mathcal{O}}
$$

and

$$
V_{2}(t, x)=v_{2}(t, x) \mathbb{1}_{E}(x)+(\ell(t)+\omega(t) \times x) \mathbb{1}_{\mathcal{O}}(x) \quad\left(t>0, x \in \mathbb{R}^{3}\right) .
$$


Recall that $U_{0} \in \operatorname{Ran}\left(\mathbb{T}_{1}^{q}\right)$, in particular $U_{0} \in \mathcal{D}\left(\mathbb{A}_{q}^{m}\right)$ for every $m \in \mathbb{N}$. Therefore $V_{20} \in \mathcal{D}\left(\mathbb{A}_{q}^{m}\right)$ for every $m \in \mathbb{N}$ and there exists a constant $C>0$, depending on $m$ and $q$, such that

$$
\left\|V_{20}\right\|_{\mathcal{D}\left(\mathbb{A}_{q}^{m}\right)} \leqslant C_{m}\left\|U_{0}\right\|_{\mathcal{D}\left(\mathbb{A}_{q}^{m}\right)} .
$$

Using (7.31), 7.32, 7.36 and 7.37), we infer that there exists a constant $C>0$, depending only on $m$ and $q$, such that

$$
\|f(t, \cdot)\|_{m, q, E} \leqslant C_{m}(1+t)^{-3 / 2 q}\|\psi\|_{[3 / q]+m+2, q} \quad(t>0) .
$$

On the other hand, applying the variation of the constants formula to 7.39, we have

$$
V_{2}(t, \cdot)=\mathbb{T}_{t}^{q} V_{20}+\int_{0}^{t} \mathbb{T}_{t-s}^{q} f(s, \cdot) \mathrm{d} s \quad(t \geqslant 0) .
$$

The last estimate, combined with Lemma 7.9, can be used, following line by line the end of the third step of the proof of Lemma 5.3 in [13], to obtain the existence of a constant $C$ (depending only on $d, m$ and $q$ ), such that for every $t>0$ we have

$$
\begin{gathered}
\left\|V_{2}(t, \cdot)\right\|_{2 m, q, E_{d}} \leqslant C_{m}(1+t)^{-3 / 2 q}\left(\left\|u_{0}\right\|_{[3 / q]+2 m+2, q, E}+\left|\ell_{0}\right|+\left|\omega_{0}\right|\right) . \\
\left\|\partial_{t} V_{2}(t, \cdot)\right\|_{2 m, q, E_{d}} \leqslant C_{m}(1+t)^{-3 / 2 q}\left(\left\|u_{0}\right\|_{[3 / q]+2 m+4, q, E}+\left|\ell_{0}\right|+\left|\omega_{0}\right|\right) .
\end{gathered}
$$

Final step. Estimates (7.26) easily follow by combining (7.38) with the estimates (7.31) - (7.33), 7.36, (7.37), 7.47) and (7.48). The estimate (7.27) can be obtained similarly. Putting together 7.26 and (7.27), from 7.19$)_{1}$ we obtain

$$
\begin{aligned}
\|\nabla \pi(t, \cdot)\|_{2 m, q, E_{d}} & \leqslant\left\|\partial_{t} u(t, \cdot)\right\|_{2 m, q, E_{d}}+\|\Delta u(t, \cdot)\|_{2 m, q, E_{d}} \\
& \leqslant C(1+t)^{-3 / 2 q}\left(\left\|u_{0}\right\|_{[3 / q]+2 m+4, q, E}+\left|\ell_{0}\right|+\left|\omega_{0}\right|\right) \quad(t \geqslant 0) .
\end{aligned}
$$

Then the estimate 7.28 follows from the above estimate after redefining $\pi$ as $\pi-\int_{E_{d}} \pi \mathrm{d} x$ and applying Poincaré type inequalities.

The results in Lemma 7.9 and Proposition 7.10 provide decay estimates for the restrictions to bounded sets of the solution $u$ of the linearized problem. The result below provides decay estimates for the restriction of $u(t, \cdot)$ to the exterior of the bounded set $E_{d}$ introduced in Lemma 7.9

Proposition 7.11. With the notation and assumptions of Remark 7.7 and Theorem 7.1, let $d>$ $R_{0}+5$. Moreover, assume that $U_{0} \in \operatorname{Ran}\left(\mathbb{T}_{1}^{q}\right)$. Then there exists a positive constant $C$, depending only on $d$ and $q$, such that, for every $t \geqslant 0$ we have

$$
\begin{aligned}
& \|u(t, \cdot)\|_{r,\{|x|>d\}} \leqslant C(1+t)^{-\sigma}\left(\left\|u_{0}\right\|_{[3 / q]+[2 \sigma]+7, q, E}+\left|\ell_{0}\right|+\left|\omega_{0}\right|\right) \quad(1<q \leqslant r<\infty), \\
& \|\nabla u(t, \cdot)\|_{r,\{|x|>d\}} \leqslant C(1+t)^{-\sigma}\left(\left\|u_{0}\right\|_{[3 / q]+[2 \sigma]+7, q, E}+\left|\ell_{0}\right|+\left|\omega_{0}\right|\right) \quad(1<q \leqslant r \leqslant 3),
\end{aligned}
$$

where $[s]$ denotes the integer part of $s \in \mathbb{R}$.

Proof. Let $\chi \in C^{\infty}\left(\mathbb{R}^{3}\right)$ be such that $\chi(x)=1$ for $|x|>d$ and $\chi(x)=0$ for $|x|<d-1$. It follows that for every $t \geqslant 0$ we have that $\operatorname{supp} \operatorname{div}(\chi u(t, \cdot)) \subset\{d-1<|x|<d\}$. Then there exists $v_{3}(t, \cdot)$ such that $\operatorname{div} v_{3}=\operatorname{div}(\chi u), \operatorname{supp} v_{3}(t, \cdot) \subset\{d-1<|x|<d\}$ and for every $m \in \mathbb{N}$, we have

$$
\begin{aligned}
& \left\|v_{3}(t, \cdot)\right\|_{m, q} \leqslant C(1+t)^{-\frac{3}{2 q}}\left(\left\|u_{0}\right\|_{[3 / q]+m+2, q, E}+\left|\ell_{0}\right|+\left|\omega_{0}\right|\right), \\
& \left\|\partial_{t} v_{3}(t, \cdot)\right\|_{m, q} \leqslant C(1+t)^{-\frac{3}{2 q}}\left(\left\|u_{0}\right\|_{[3 / q]+m+4, q, E}+\left|\ell_{0}\right|+\left|\omega_{0}\right|\right),
\end{aligned}
$$


for some constant $C>0$ depending on $m$ and $q$. To derive the last two estimates we have used Bogovskii Lemma, (7.26) and (7.27). We now define

$$
v_{4}(t, x)=\chi(x) u(t, x)-v_{3}(t, x) \quad\left(t>0, x \in \mathbb{R}^{3}\right) .
$$

Note that $\operatorname{div} v_{4}=0$ so that $v_{4}$ satisfies

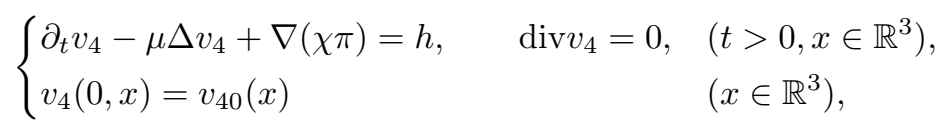

where

$$
h=-2(\nabla \chi \cdot \nabla) u(t)-\mu(\Delta \chi) u+\partial_{t} v_{3}-\mu \Delta v_{3}+\pi \nabla \chi
$$

and

$$
v_{40}(x)=\chi(x) U_{0}(x)-v_{3}(0, x) \quad\left(x \in \mathbb{R}^{3}\right) .
$$

Note that, since all the functions appearing in 7.54 are supported away from $\mathcal{O}$, the function $v_{4}$ shares all the properties derived in Lemma 5.5 and proofs of Theorems 1.2 and 1.3 of [13]. In particular,

$$
\left\|v_{4}(t, \cdot)\right\|_{r} \leqslant C(1+t)^{-\sigma}\left(\left\|u_{0}\right\|_{[3 / q]+[2 \sigma]+7, q, E}+\left|\ell_{0}\right|+\left|\omega_{0}\right|\right) \quad(1<q \leqslant r<\infty)
$$

and

$$
\left\|\nabla v_{4}(t, \cdot)\right\|_{r} \leqslant C(1+t)^{-\sigma-1 / 2}\left(\left\|u_{0}\right\|_{[3 / q]+[2 \sigma]+7, q, E}+\left|\ell_{0}\right|+\left|\omega_{0}\right|\right) \quad(1<q \leqslant r \leqslant 3) .
$$

By combining (7.57), 7.51) (with $m=[2 \sigma]+1$ ) and Sobolev's embedding theorem we conclude that

$$
\begin{aligned}
& \|u(t)\|_{r,\{|x|>d\}} \leqslant\left\|v_{3}(t)\right\|_{r, E}+\left\|v_{4}(t)\right\|_{r} \\
& \leqslant C\left\|v_{3}(t)\right\|_{[2 \sigma]+1, q, E}+\left\|v_{4}(t)\right\|_{r} \\
& \leqslant C(1+t)^{-\frac{3}{2 q}}\left(\left\|u_{0}\right\|_{[3 / q]+m+3, q, E}+\left|\ell_{0}\right|+\left|\omega_{0}\right|\right)+C(1+t)^{-\sigma}\left(\left\|u_{0}\right\|_{[3 / q]+m+7, q, E}+\left|\ell_{0}\right|+\left|\omega_{0}\right|\right) \\
& \leqslant C(1+t)^{-\sigma}\left(\left\|u_{0}\right\|_{[3 / q]+m+7, q, E}+\left|\ell_{0}\right|+\left|\omega_{0}\right|\right)
\end{aligned}
$$

This completes the proof of $(7.49)$.

Finally, the proof of 7.50 is obtained similarly from (7.58), together with (7.51) (with $m=$ $[2 \sigma]+2)$.

We are now in a position to prove the main result in this section.

Proof of Theorem 7.1, items (i)-(iii). For small times, Theorem 7.1 items (i)-(iii) simply is Theorem 7.8, and we thus only focus on the estimates of Theorem 7.1 items (i)-(iii) for times larger than 1 .

To prove 7.1 , it suffices to note that, for every $U \in \mathbb{X}^{q}$ we have $\mathbb{T}_{1}^{q} U \in \mathcal{D}\left(\mathbb{A}_{q}^{k}\right)$ for any $k \in \mathbb{N}$, so that applying $(7.26)$ with $m=0$ we obtain

$$
\left\|\mathbb{T}_{t+1}^{q} U\right\|_{q, B_{R}} \leqslant C(1+t)^{-3 / 2 q}\|U\|_{q, \mathbb{R}^{3}} \quad\left(t>0, U \in \mathbb{X}^{q}\right) .
$$

Concerning 7.2 , we first note that this estimate holds for $t \in(0,1]$ (see 7.20$)$ ). Again applying 7.26 with $m=[2 \sigma]+1$, we get

$$
\left\|\mathbb{T}_{t+1}^{q} U\right\|_{r, E_{d}} \leqslant C\left\|\mathbb{T}_{t+1}^{q} U\right\|_{[2 \sigma]+1, q, E_{d}} \leqslant C(1+t)^{-3 / 2 q}\|U\|_{q, \mathbb{R}^{3}} \quad\left(t>0, U \in \mathbb{X}^{q}\right),
$$


and by 7.49

$$
\left\|\mathbb{T}_{t+1}^{q} U\right\|_{r,\{|x|>d\}} \leqslant C(1+t)^{-\sigma}\|U\|_{q, \mathbb{R}^{3}} \quad\left(t>0, U \in \mathbb{X}^{q}\right) .
$$

The above two estimates give 7.2 for $t \geqslant 1$.

The proof of 7.3 follows analogously by combining (7.21, (7.26) and 7.50).

To complete the proof of Theorem 7.1 it remains to show that 7.2 holds for $r=\infty$ and $1<q<\infty$. To this aim, we first prove the following result

Proposition 7.12. With the notation and assumptions of Remark 7.7 and Theorem 7.1, let $d>$ $R_{0}+5$. Moreover, assume that $q>3$ and $U_{0} \in \operatorname{Ran}\left(\mathbb{T}_{1}^{q}\right)$. Then there exists a positive constant $C$, depending only on $d$ and $q$, such that, for every $t \geqslant 0$ we have

$$
\|u\|_{\infty, E} \leqslant C(1+t)^{-\frac{3}{2 q}}\left(\left\|u_{0}\right\|_{[3 / q]+6, q, E}+\left|\ell_{0}\right|+\left|\omega_{0}\right|\right), \quad(t \geqslant 0, \quad 3<q<\infty) .
$$

where $[s]$ denotes the integer part of $s \in \mathbb{R}$.

Proof. Combining (7.38) together with (7.32, 7.36) and 7.47) for $m=1$, we deduce that

$$
\|u\|_{\infty, E_{d}} \leqslant C(1+t)^{-3 / 2 q}\left(\left\|u_{0}\right\|_{[3 / q]+6, q, E}+\left|\ell_{0}\right|+\left|\omega_{0}\right|\right),
$$

where the set $E_{d}$ has been defined in Lemma 7.9. Moreover, from (7.51) we also have

$$
\left\|v_{3}(t, \cdot)\right\|_{\infty} \leqslant C(1+t)^{-\frac{3}{2 q}}\left(\left\|u_{0}\right\|_{[3 / q]+6, q, E}+\left|\ell_{0}\right|+\left|\omega_{0}\right|\right),
$$

where $v_{3}$ is defined as in Proposition 7.11. Therefore, by virtue of the decomposition (7.53), it remains to show the $L^{\infty}$ estimate of $v_{4}$, where $v_{4}$ is defined by (7.54). Recall the definition of $h$ from (7.55). Using (7.26, 7.28, ,7.51) and (7.52, we obtain for any $m \in \mathbb{N} \cup\{0\}$

$$
\|h(t, \cdot)\|_{m, q} \leqslant C(1+t)^{-\frac{3}{2 q}}\left(\left\|u_{0}\right\|_{[3 / q]+m+4, q, E}+\left|\ell_{0}\right|+\left|\omega_{0}\right|\right), \quad(t \geqslant 0) .
$$

Let us take $q_{0} \in(1,3 / 2)$. Then by using 7.32 and the above estimate, we evaluate

$$
\begin{aligned}
\left\|v_{4}\right\|_{\infty} & \leqslant C\left((1+t)^{-3 / 2 q}\left\|v_{40}\right\|_{[3 / q]+1, q}+\int_{0}^{t}(1+t-s)^{-3 / 2 q_{0}}\|h(s)\|_{\left[3 / q_{0}\right]+1, q} \mathrm{~d} s\right) \\
& \leqslant C(1+t)^{-\frac{3}{2 q}}\left(\left\|u_{0}\right\|_{[3 / q]+6, q, E}+\left|\ell_{0}\right|+\left|\omega_{0}\right|\right)\left(1+\int_{0}^{t} \frac{(1+t)^{3 / 2 q}}{(1+t-s)^{3 / 2 q_{0}}(1+s)^{3 / 2 q}} \mathrm{~d} s\right) \\
& \leqslant C(1+t)^{-\frac{3}{2 q}}\left(\left\|u_{0}\right\|_{[3 / q]+6, q, E}+\left|\ell_{0}\right|+\left|\omega_{0}\right|\right) .
\end{aligned}
$$

The above estimate together with 7.61) and 7.62 implies 7.60).

Proof of Theorem 7.1, item (iv), estimate (7.2) for $q \in(1, \infty)$ and $r=\infty$. By (7.59) and (7.60) we have

$$
\left\|\mathbb{T}_{t+1}^{q} U\right\|_{\infty} \leqslant C(1+t)^{-3 / 2 q}\|U\|_{\mathbb{X}^{q}} \quad\left(t>0,3<q<\infty, U \in \mathbb{X}^{q}\right) .
$$

This proves 7.2 for $q \in(3, \infty), r=\infty$, and $t \geqslant 1$. Let $\tau \in(0, \infty)$. For $t \in(0, \tau), 1<q<\infty$, and $q_{0}>\max \{3, q\}$, we apply Theorem 7.8 to obtain

$$
\left\|\mathbb{T}_{t}^{q} U\right\|_{\infty, E} \leqslant C\left\|\nabla \mathbb{T}_{t}^{q} U\right\|_{q_{0}, E}^{3 / q_{0}}\left\|\mathbb{T}_{t}^{q} U\right\|_{q_{0}, E}^{1-3 / q_{0}} \leqslant C_{\tau} t^{-3 / 2 q}\|U\|_{\mathbb{X} q},
$$

where the constant $C_{\tau}$ depends on $\tau$ but independent of $t$.

Finally, for $t \geqslant 2$ and $q \leqslant 3$, we choose $q_{0}>3$ and apply $(7.2)$ and the above estimates to obtain

$$
\left\|\mathbb{T}_{t}^{q} U\right\|_{\mathbb{X}^{\infty}} \leqslant C t^{-3 / 2 q_{0}}\left\|\mathbb{T}_{t / 2}^{q} U\right\|_{\mathbb{X}^{q_{0}}} \leqslant C t^{-3 / 2 q}\|U\|_{\mathbb{X}^{q}}
$$

This completes the proof of item (iv) of Theorem 7.1 


\section{Proof of the main results}

In this section, we focus on the analysis of the non-linear fluid-structure model, assuming that the rigid body is a ball. Note that this assumption has been already used when fixing the frame via a simple translation, which drastically simplifies the structure of the non-linear terms. Given $q>1$ we continue to use the notation $\mathbb{X}^{q}$ for the space defined in Eq. (3.1) and $\mathbb{T}^{q}$ for the fluid structure semigroup introduced in the previous sections. However, to simplify the notation and when there is no risk of confusion, the fluid-structure semigroup will be simply denoted by $\mathbb{T}$. Similarly, if the appropriate value of $q$ is clear from the context, the projector $\mathbb{P}_{q}$, introduced in Proposition 3.2 , is simply denoted by $\mathbb{P}$.

The arguments we are using are close to those in Kato [14, with several adaptations necessary to tackle the extra term coming from the motion of the rigid body, in a spirit close to [6], and with the extensive use of the results obtained in the previous sections on the fluid-structure semigroup, and in particular Theorem 7.1 .

We rely, in particular, on the following lemma, which is a rather straightforward consequence of Theorem 7.1 :

Lemma 8.1. Let $p_{0}$ and $q_{0}$ be such that $q_{0} \in[3 / 2, \infty)$ and $p_{0} \in\left[q_{0}, \infty\right]$. Then there exists $C>0$ such that for every $F \in L^{q_{0}}\left(\mathbb{R}^{3} ; \mathbb{R}^{3 \times 3}\right)$ satisfying $F=0$ in $B$ and $\operatorname{div} F \in\left[L^{r}\left(\mathbb{R}^{3}\right)\right]^{3}$ for some $r \in\left(1, p_{0}\right] \backslash\{\infty\}$ we have

$$
\left\|\mathbb{T}_{t}^{r} \mathbb{P}_{r} \operatorname{div} F\right\|_{p_{0}} \leqslant C t^{-3 / 2\left(1 / q_{0}-1 / p_{0}\right)-1 / 2}\|F\|_{q_{0}, E} \quad(t>0) .
$$

Proof. The proof follows the same steps as those appearing in [6. Proof of Corollary 3.10]. More precisely, for $F \in L^{q_{0}}\left(\mathbb{R}^{3} ; \mathbb{R}^{3 \times 3}\right)$ satisfying $F=0$ in $B$ and $\operatorname{div} F \in\left[L^{r}\left(\mathbb{R}^{3}\right)\right]^{3}$ for some $r \in$ $\left(1, p_{0}\right] \backslash\{\infty\}$, we necessarily have $F \cdot \nu=0$ on $\partial B$ and for $t>0, \mathbb{T}_{t}^{r} \mathbb{P}_{r} \operatorname{div} F$ is a well-defined element of $\mathbb{X}^{r} \cap \mathbb{X}^{\infty}$ (see Theorem 7.1).

Setting $r_{0}=\max \left\{r, q_{0}\right\}$, we thus have that

$$
\begin{aligned}
& \left\|\mathbb{T}_{t}^{r} \mathbb{P}_{r} \operatorname{div}(F)\right\|_{r_{0}} \\
& =\sup _{\varphi \in \mathbb{X}^{r_{0}^{\prime}},\|\varphi\|_{\mathbb{X}^{r_{0}^{\prime}}} \leqslant 1}\left\{\left\langle\mathbb{T}_{t}^{r} \mathbb{P}_{r} \operatorname{div}(F), \varphi\right\rangle_{\left.\mathbb{X}^{r_{0}, \mathbb{X}^{r_{0}^{\prime}}}\right\}}\left\{\left\langle\operatorname{div} F, \mathbb{T}_{t}^{r^{\prime}} \varphi\right\rangle_{\mathbb{X}^{r}, \mathbb{X}^{r^{\prime}}}\right\} \quad \text { ( by Proposition } 3.1 \text { and Proposition } 5.3\right) \\
& =\sup _{\varphi \in \mathbb{X}^{r_{0}^{\prime}},\|\varphi\|_{\mathbb{X}^{r_{0}^{\prime}}} \leqslant 1}\left\{-\int_{E} F \cdot \nabla \mathbb{T}_{t}^{r_{0}^{\prime}} \varphi \mathrm{d} x\right\} \quad(\text { as } F=0 \text { in } B) \\
& =\sup _{\varphi \in \mathbb{X}^{r_{0}^{\prime}},\|\varphi\|_{\mathbb{X}^{r_{0}^{\prime}}} \leqslant 1}\left\{\|F\|_{q_{0}, E} \sup _{\varphi \in \mathbb{X}^{r_{0}^{\prime}},\|\varphi\|_{\mathbb{X}_{0} r_{0}^{\prime}} \leqslant 1}\left\|\nabla \mathbb{T}_{t}^{r_{0}^{\prime}} \varphi\right\|_{q_{0}^{\prime}, E} .\right.
\end{aligned}
$$

Finally, using 7.3 , we obtain

$$
\left\|\mathbb{T}_{t}^{r} \mathbb{P}_{r} \operatorname{div} F\right\|_{r_{0}} \leqslant C t^{-3 / 2\left(1 / q_{0}-1 / r_{0}\right)-1 / 2}\|F\|_{q_{0}, E} \quad(t>0) .
$$

Now, for $p_{0} \in\left[r_{0}, \infty\right]$, we use

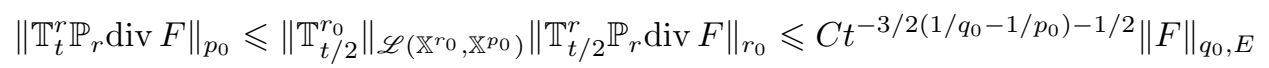

where the last estimate comes from 7.2 .

We state below a result which, taking in consideration the notation recalled at the beginning of this section, clearly includes our main result in Theorem 1.1. More precisely, this result provides 
local in time existence of solutions for initial data in $\mathbb{X}^{3}$ and global existence of solutions of $(1.2)$ for small data in $\mathbb{X}^{3}$, with a description of the long time behavior of the solutions when further assuming that the initial datum belongs to $\mathbb{X}^{q}$ for some $q \in(1,3]$.

Theorem 8.2. Let $V_{0} \in \mathbb{X}^{3}$. Then there exists $T_{0}>0$ such that there exists a unique solution $V=\left[\begin{array}{l}v \\ \ell \\ \omega\end{array}\right] \in C^{0}\left(\left[0, T_{0}\right] ; \mathbb{X}^{3}\right)$ with $t^{1 / 4} V(t) \in C^{0}\left(\left[0, T_{0}\right] ; \mathbb{X}^{6}\right), t^{1 / 2} V(t) \in C^{0}\left(\left[0, T_{0}\right] ; \mathbb{X}^{\infty}\right)$ and $\min \left\{t^{1 / 2}, 1\right\} \nabla v(t) \in C^{0}\left(\left[0, T_{0}\right] ;\left[L^{3}(E)\right]^{9}\right)$ of $[1.2$, such that

$$
\lim _{t \rightarrow 0}\left(\left\|t^{1 / 4} V(t)\right\|_{\mathbb{X}^{6}}+\left\|t^{1 / 2} V(t)\right\|_{\mathbb{X}^{\infty}}+\left\|t^{1 / 2} \nabla v(t)\right\|_{3, E}\right)=0 .
$$

Furthermore, this solution is such that for all $p \in[3, \infty], t^{3 / 2(1 / 3-1 / p)} V \in C^{0}\left(\left[0, T_{0}\right] ; \mathbb{X}^{p}\right)$.

Besides, there exists $\varepsilon_{0}>0$ such that if $\left\|V_{0}\right\|_{3} \leqslant \varepsilon_{0}, T_{0}$ can be taken to be infinite, i.e. $T_{0}=\infty$.

For $q \in(1,3]$, there exists $\varepsilon_{0}(q) \in\left(0, \varepsilon_{0}\right]$ such that if $V_{0}$ belongs to $\mathbb{X}^{q} \cap \mathbb{X}^{3}$ and satisfies $\left\|V_{0}\right\|_{3} \leqslant \varepsilon_{0}(q)$, then the solution $V$ also satisfies, for all $p \in[\max \{q, 3 / 2\}, \infty], t^{3 / 2(1 / q-1 / p)} V \in$ $C_{b}^{0}\left([0, \infty] ; \mathbb{X}^{p}\right)$.

Proof. Existence theory for $V_{0} \in \mathbb{X}^{3}$. We first focus on the existence of solutions $V$ of 1.2 .

As mentioned in the introduction, we are looking for mild solutions $V$ of the non-linear problem (1.2), i.e. solutions of the equation 1.7 . For each $t>0$ we identify $V(t, \cdot)$ with a triple $\left[\begin{array}{c}v(t, \cdot) \\ \ell(t) \\ \omega(t)\end{array}\right]$, where $v(t, \cdot): E \rightarrow \mathbb{R}^{3}$ and $\ell(t), \omega(t) \in \mathbb{R}^{3}$, as described in 3.3 .

We first remark that, since $(v-\ell) \cdot \nu=0$ on $\partial B$, we have

$$
-\mathbb{1}_{E}[(v-\ell) \cdot \nabla] v=\operatorname{div} F
$$

where

$$
F(s, x)=-\mathbb{1}_{E}(x)(v(s, x)-\ell(s)) \otimes v(s, x) \quad\left(s>0, x \in \mathbb{R}^{3}\right) .
$$

In particular the triple $V=\left[\begin{array}{l}v \\ \ell \\ \omega\end{array}\right]$ is a mild solution of 1.2 iff it satisfies

$$
V(t)=\mathbb{T}_{t} V_{0}+\int_{0}^{t} \mathbb{T}_{t-s} \mathbb{P} \operatorname{div} F(s) \mathrm{d} s \quad(t \geqslant 0),
$$

where $F$ is defined in 8.3 . The above formulation will be intensively used in the remaining part of the section, in conjonction with Lemma 8.1 .

For $T>0$, we introduce the class

$$
\begin{array}{r}
\mathscr{C}(T)=\left\{\begin{array}{r}
v \\
\ell \\
\omega
\end{array}\right] \quad \text { with } t^{1 / 4} V \in C^{0}\left([0, T] ; \mathbb{X}^{6}\right), t^{1 / 2} V \in C^{0}\left([0, T] ; \mathbb{X}^{\infty}\right) \\
\text { and } \left.\min \left\{t^{1 / 2}, 1\right\} \nabla v \in C^{0}\left([0, T] ;\left[L^{3}(E)\right]^{9}\right)\right\},
\end{array}
$$

which we endow with the norm

$$
\|V\|_{\mathscr{C}(T)}=\left\|t^{1 / 4} V(t)\right\|_{L^{\infty}\left(0, T ; \mathbb{X}^{6}\right)}+\left\|t^{1 / 2} V(t)\right\|_{L^{\infty}\left(0, T ; \mathbb{X}^{\infty}\right)}+\left\|\min \left\{t^{1 / 2}, 1\right\} \nabla v\right\|_{L^{\infty}\left([0, T] ;\left[L^{3}(E)\right]^{9}\right)} .
$$


Note in particular that, for $V=\left[\begin{array}{l}v \\ \ell \\ \omega\end{array}\right] \in \mathscr{C}(T)$, we have the estimate

$$
|\ell(t)| \leqslant \frac{1}{\max \left\{t^{1 / 4}, t^{1 / 2}\right\}}\|V\|_{\mathscr{C}(T)}, \quad(t \geqslant 0) .
$$

We start by remarking that we are looking for a solution $V$ of 1.7 or equivalently (8.4). We then define the map $\Lambda_{T}: V \in \mathscr{C}(T) \mapsto \Lambda_{T} V$ defined for $t \in[0, T]$ by

$$
\Lambda_{T} V(t)=\mathbb{T}_{t} V_{0}+\int_{0}^{t} \mathbb{T}_{t-s} \mathbb{P}\left(\mathbb{1}_{E}(\ell(s)-v(s)) \cdot \nabla v(s)\right) d s,
$$

or, equivalently,

$$
\Lambda_{T} V(t)=\mathbb{T}_{t} V_{0}+\int_{0}^{t} \mathbb{T}_{t-s} \mathbb{P} \operatorname{div}\left(\mathbb{1}_{E}(\ell(s)-v(s)) \otimes v(s)\right) d s,
$$

Next we claim the following lemma:

Lemma 8.3. There exists a constant $C_{0}>0$ independent of $T$ such that

$$
\begin{array}{ll}
\left\|\Lambda_{T} V\right\|_{\mathscr{C}(T)} \leqslant\left\|\mathbb{T}_{t} V_{0}\right\|_{\mathscr{C}(T)}+C_{0}\|V\|_{\mathscr{C}(T)}^{2}, & (V \in \mathscr{C}(T)), \\
\left\|\Lambda_{T} V^{a}-\Lambda_{T} V^{b}\right\|_{\mathscr{C}(T)} \leqslant C_{0}\left(\left\|V^{a}\right\|_{\mathscr{C}(T)}+\left\|V^{a}\right\|_{\mathscr{C}(T)}\right)\left\|V^{a}-V^{b}\right\|_{\mathscr{C}(T)}, & \left(V^{a}, V^{b} \in \mathscr{C}(T)\right) .
\end{array}
$$

Proof. Since estimate (8.6) can be easily deduced from (8.7) by taking $V^{a}=0$ and $V^{b}=V$, we prove only 8.7).

Let $V^{a}$ and $V^{b}$ be in $\mathscr{C}(T)$. Then easy computations show that

$$
\Lambda_{T} V^{a}(t)-\Lambda_{T} V^{b}(t)=\int_{0}^{t} \mathbb{T}_{t-s} \mathbb{P} \operatorname{div} G(s) d s,
$$

where $G$ is given by

$$
G(s)=\mathbb{1}_{E}\left(\left(\ell^{a}(s)-v^{a}(s)\right) \otimes v^{a}(s)-\left(\ell^{b}(s)-v^{b}(s)\right) \otimes v^{b}(s)\right), \quad(s \in(0, T]) .
$$

Writing

$$
\begin{aligned}
G(s)=\mathbb{1}_{E}\left(\left(\ell^{a}(s)-\ell^{b}(s)\right)-\left(v^{a}(s)-v^{b}(s)\right)\right) \otimes v^{a}(s) & \\
& +\mathbb{1}_{E}\left(\ell^{b}(s)-v^{b}(s)\right) \otimes\left(v^{a}(s)-v^{b}(s)\right), \quad(s \in(0, T]),
\end{aligned}
$$

we easily deduce that for all $s \in(0, T)$,

$$
\begin{aligned}
\|G(s)\|_{6} & \leqslant 2\left\|V^{a}(s)-V^{b}(s)\right\|_{\infty}\left(\left\|V^{a}(s)\right\|_{6}+\left\|V^{b}(s)\right\|_{6}\right)+2\left\|V^{a}(s)-V^{b}(s)\right\|_{6}\left(\left\|V^{a}(s)\right\|_{\infty}+\left\|V^{b}(s)\right\|_{\infty}\right) \\
& \leqslant \frac{2}{s^{3 / 4}}\left(\left\|V^{a}\right\|_{\mathscr{C}(T)}+\left\|V^{b}\right\|_{\mathscr{C}(T)}\right)\left\|V^{a}-V^{b}\right\|_{\mathscr{C}(T)} .
\end{aligned}
$$

Besides, for all $s \in(0, T] \operatorname{div} G(s)$ belongs to $\left[L^{3}\left(\mathbb{R}^{3}\right)\right]^{3}$ since $V^{a}$ and $V^{b}$ belongs to $\mathscr{C}(T)$. Therefore, using Lemma 8.1, with $p_{0}=q_{0}=6$,

$$
\begin{aligned}
& \left\|t^{1 / 4}\left(\Lambda_{T} V^{a}(t)-\Lambda_{T} V^{b}(t)\right)\right\|_{L^{\infty}\left(0, T ; \mathbb{X}^{6}\right)} \\
& \leqslant C \sup _{t \in(0, T]}\left\{t^{1 / 4} \int_{0}^{t} \frac{1}{(t-s)^{1 / 2}} \frac{1}{s^{3 / 4}} d s\right\}\left(\left\|V^{a}\right\|_{\mathscr{C}(T)}+\left\|V^{b}\right\|_{\mathscr{C}(T)}\right)\left\|V^{a}-V^{b}\right\|_{\mathscr{C}(T)} \\
& \leqslant C\left(\left\|V^{a}\right\|_{\mathscr{C}(T)}+\left\|V^{b}\right\|_{\mathscr{C}(T)}\right)\left\|V^{a}-V^{b}\right\|_{\mathscr{C}(T)},
\end{aligned}
$$


for some $C$ independent of $T$, where we used that, by scaling, for all $t>0$,

$$
t^{1 / 4} \int_{0}^{t} \frac{1}{(t-s)^{1 / 2} s^{3 / 4}} d s=\int_{0}^{1} \frac{1}{(1-s)^{1 / 2} s^{3 / 4}} d s .
$$

Similarly, using again Lemma 8.1. with $p_{0}=\infty, q_{0}=6$, we get, for some $C$ independent of $T$,

$$
\begin{aligned}
& \left\|t^{1 / 2}\left(\Lambda_{T} V^{a}(t)-\Lambda_{T} V^{b}(t)\right)\right\|_{L^{\infty}\left(0, T ; \mathbb{X}^{\infty}\right)} \\
& \leqslant C \sup _{t \in(0, T]}\left\{t^{1 / 2} \int_{0}^{t} \frac{1}{(t-s)^{3 / 4}} \frac{1}{s^{3 / 4}} d s\right\}\left(\left\|V^{a}\right\|_{\mathscr{C}(T)}+\left\|V^{b}\right\|_{\mathscr{C}(T)}\right)\left\|V^{a}-V^{b}\right\|_{\mathscr{C}(T)} \\
& \leqslant C\left(\left\|V^{a}\right\|_{\mathscr{C}(T)}+\left\|V^{b}\right\|_{\mathscr{C}(T)}\right)\left\|V^{a}-V^{b}\right\|_{\mathscr{C}(T)} .
\end{aligned}
$$

To estimate $\nabla\left(\Lambda_{T} V^{a}-\Lambda_{T} V^{b}\right)$, it is convenient to first write

$$
\Lambda_{T} V^{a}(t)-\Lambda_{T} V^{b}(t)=\int_{0}^{t} \mathbb{T}_{t-s} \mathbb{P} g(s) d s, \quad(t \in(0, T]),
$$

where $g$ is given

$$
\begin{aligned}
& g(s)=\mathbb{1}_{E}\left(\left(\ell^{a}(s)-\ell^{b}(s)\right) \cdot \nabla v^{a}(s)+\ell^{b}(s) \cdot \nabla\left(v^{a}(s)-v^{b}(s)\right)\right) \\
& \left.\quad-\mathbb{1}_{E}\left(v^{a}(s)-v^{b}(s)\right)\right) \cdot \nabla v^{a}(s)-\mathbb{1}_{E} v^{b}(s) \cdot \nabla\left(v^{a}(s)-v^{b}(s)\right), \quad(s \in(0, T]),
\end{aligned}
$$

that we decompose as

$$
g(s)=g_{\ell}(s)+g_{v}(s), \quad(s \in(0, T]),
$$

with

$$
\begin{aligned}
& g_{\ell}(s)=\mathbb{1}_{E}\left(\left(\ell^{a}(s)-\ell^{b}(s)\right) \cdot \nabla v^{a}(s)+\mathbb{1}_{E} \ell^{b}(s) \cdot \nabla\left(v^{a}(s)-v^{b}(s)\right), \quad(s \in(0, T]),\right. \\
& \left.g_{v}(s)=-\mathbb{1}_{E}\left(v^{a}(s)-v^{b}(s)\right)\right) \cdot \nabla v^{a}(s)-\mathbb{1}_{E} v^{b}(s) \cdot \nabla\left(v^{a}(s)-v^{b}(s)\right), \quad(s \in(0, T]) .
\end{aligned}
$$

We then bound $g_{\ell}$ in $\left[L^{3}\left(\mathbb{R}^{3}\right)\right]^{9}:$ for $s \in(0, T]$,

$$
\begin{aligned}
\left\|g_{\ell}(s)\right\|_{3} & \leqslant\left|\ell^{a}(s)-\ell^{b}(s)\right|\left\|\nabla v^{a}(s)\right\|_{3, E}+\left|\ell^{b}(s)\right|\left\|\nabla\left(v^{a}-v^{b}\right)(s)\right\|_{3, E} \\
& \leqslant \frac{2}{\max \left\{s^{1 / 4}, s^{1 / 2}\right\} \min \left\{1, s^{1 / 2}\right\}}\left(\left\|V^{a}\right\|_{\mathscr{C}(T)}+\left\|V^{b}\right\|_{\mathscr{C}(T)}\right)\left\|V^{a}-V^{b}\right\|_{\mathscr{C}(T)} \\
& \leqslant \frac{2}{\min \left\{s^{3 / 4}, s^{1 / 2}\right\}}\left(\left\|V^{a}\right\|_{\mathscr{C}(T)}+\left\|V^{b}\right\|_{\mathscr{C}(T)}\right)\left\|V^{a}-V^{b}\right\|_{\mathscr{C}(T)} .
\end{aligned}
$$

We also bound $g_{v}$ in $\left[L^{2}\left(\mathbb{R}^{3}\right)\right]^{9}$ : for $s \in(0, T]$,

$$
\begin{aligned}
\left\|g_{v}(s)\right\|_{2} & \leqslant\left\|V^{a}(s)-V^{b}(s)\right\|_{\mathbb{X}^{6}}\left\|\nabla v^{a}(s)\right\|_{3, E}+\left\|V^{b}(s)\right\|_{\mathbb{X}^{6}}\left\|\nabla\left(v^{a}-v^{b}\right)(s)\right\|_{3, E} \\
& \leqslant \frac{2}{s^{1 / 4} \min \left\{1, s^{1 / 2}\right\}}\left(\left\|V^{a}\right\|_{\mathscr{C}(T)}+\left\|V^{b}\right\|_{\mathscr{C}(T)}\right)\left\|V^{a}-V^{b}\right\|_{\mathscr{C}(T)} \\
& \leqslant \frac{2}{\min \left\{s^{3 / 4}, s^{1 / 4}\right\}}\left(\left\|V^{a}\right\|_{\mathscr{C}(T)}+\left\|V^{b}\right\|_{\mathscr{C}(T)}\right)\left\|V^{a}-V^{b}\right\|_{\mathscr{C}(T)} .
\end{aligned}
$$

Accordingly, estimate 7.3 yields

$$
\begin{aligned}
\left\|\min \left\{1, t^{1 / 2}\right\} \nabla\left(\Lambda_{T} V^{a}(t)-\Lambda_{T} V^{b}(t)\right)\right\|_{L^{\infty}\left(0, T ;\left[L^{3}(E)\right]^{9}\right)} & \\
\leqslant C \sup _{t \in[0, T]}\left\{\int_{0}^{t} \frac{\min \left\{1, t^{1 / 2}\right\}}{(t-s)^{1 / 2} \min \left\{s^{3 / 4}, s^{1 / 2}\right\}} d s\right. & \left.+\int_{0}^{t} \frac{\min \left\{1, t^{1 / 2}\right\}}{(t-s)^{3 / 4} \min \left\{s^{3 / 4}, s^{1 / 4}\right\}} d s\right\} \\
& \times\left(\left\|V^{a}\right\|_{\mathscr{C}(T)}+\left\|V^{b}\right\|_{\mathscr{C}(T)}\right)\left\|V^{a}-V^{b}\right\|_{\mathscr{C}(T)} .
\end{aligned}
$$


We then show that the supremum in $t \in[0, T]$ can in fact be bounded by a constant independent of $T$. For $t \in(0,1)$,

$$
\begin{aligned}
& \int_{0}^{t} \frac{\min \left\{1, t^{1 / 2}\right\}}{(t-s)^{1 / 2} \min \left\{s^{3 / 4}, s^{1 / 2}\right\}} d s=\int_{0}^{t} \frac{t^{1 / 2}}{(t-s)^{1 / 2} s^{3 / 4}} d s=c_{1 / 2,3 / 4} t^{1 / 4} \leqslant c_{1 / 2,3 / 4}, \\
& \int_{0}^{t} \frac{\min \left\{1, t^{1 / 2}\right\}}{(t-s)^{3 / 4} \min \left\{s^{3 / 4}, s^{1 / 4}\right\}} d s=\int_{0}^{t} \frac{t^{1 / 2}}{(t-s)^{3 / 4} s^{3 / 4}} d s=c_{3 / 4,3 / 4},
\end{aligned}
$$

where $c_{\alpha, \beta}$ is defined for $(\alpha, \beta) \in(0,1)^{2}$ by

$$
c_{\alpha, \beta}=\int_{0}^{1} \frac{1}{(1-s)^{\alpha} s^{\beta}} d s .
$$

For $t \geqslant 1$, we write

$$
\begin{aligned}
& \int_{0}^{t} \frac{\min \left\{1, t^{1 / 2}\right\}}{(t-s)^{1 / 2} \min \left\{s^{3 / 4}, s^{1 / 2}\right\}} d s \\
& =\int_{0}^{1} \frac{1}{(t-s)^{1 / 2} s^{3 / 4}} d s+\int_{1}^{t} \frac{1}{(t-s)^{1 / 2} s^{1 / 2}} d s \leqslant c_{1 / 2,3 / 4}+c_{1 / 2,1 / 2} \\
& \int_{0}^{t} \frac{\min \left\{1, t^{1 / 2}\right\}}{(t-s)^{3 / 4} \min \left\{s^{3 / 4}, s^{1 / 4}\right\}} d s \\
& =\int_{0}^{1} \frac{1}{(t-s)^{3 / 4} s^{3 / 4}} d s+\int_{1}^{t} \frac{1}{(t-s)^{3 / 4} s^{1 / 4}} d s \leqslant c_{3 / 4,3 / 4}+c_{3 / 4,1 / 4}
\end{aligned}
$$

Consequently, there exists some $C$ independent of $T>0$ such that

$$
\begin{aligned}
\left\|\min \left\{1, t^{1 / 2}\right\} \nabla\left(\Lambda_{T} V^{a}(t)-\Lambda_{T} V^{b}(t)\right)\right\|_{L^{\infty}\left(0, T ;\left[L^{3}(E)\right]^{9}\right)} & \\
& \leqslant C\left(\left\|V^{a}\right\|_{\mathscr{C}(T)}+\left\|V^{b}\right\|_{\mathscr{C}(T)}\right)\left\|V^{a}-V^{b}\right\|_{\mathscr{C}(T)} .
\end{aligned}
$$

Putting together estimates (8.8), (8.9) and 8.14), we conclude the estimate 8.7 and Lemma 8.3 .

According to Lemma 8.3. for $K>0$, the set $\mathscr{C}(T, K)=\left\{V \in \mathscr{C}(T),\|V\|_{\mathscr{C}(T)} \leqslant K\right\}$ is such that for all $V^{a}$ and $V^{b}$ in $\mathscr{C}(T, K)$,

$$
\left\|\Lambda_{T} V^{a}-\Lambda_{T} V^{b}\right\|_{\mathscr{C}(T)} \leqslant 2 C_{0} K\left\|V^{a}-V^{b}\right\|_{\mathscr{C}(T)},
$$

where $C_{0}$ is the constant in Lemma 8.3. Therefore, for $K \leqslant K_{0}=1 /\left(4 C_{0}\right)$, the map $\Lambda_{T}$ is 1/2-Lipschitz in $\mathscr{C}(T, K)$.

Now, for $V_{0} \in \mathbb{X}^{3}$, the map $T \mapsto\left\|\mathbb{T}_{t} V_{0}\right\|_{\mathscr{C}(T)}$ is a continuous increasing function of $T$, which is bounded by $C\left\|V_{0}\right\|_{\mathbb{X}^{3}}$ according to estimates $(7.2)-(7.3)$, and which goes to 0 as $T$ to 0 by density of $\mathcal{D}\left(A_{3}\right)$ in $\mathbb{X}^{3}$ and the decay estimates $(7.2)-(7.3)$.

Therefore, for $V_{0} \in \mathbb{X}^{3}$, we can guarantee that there exists a time $T_{K}>0$ such that

$$
\left\|\mathbb{T}_{t} V_{0}\right\|_{\mathscr{C}\left(T_{K}\right)} \leqslant \frac{K}{2}
$$

so that by 8.6), for $K \leqslant K_{0}=1 /\left(4 C_{0}\right)$, the set $\mathscr{C}\left(T_{K}, K\right)$ is stable by $\Lambda_{T_{K}}$, and $\Lambda_{T_{K}}$ is strictly contractive in it. Therefore, by Banach-Picard fixed point theorem, there exists a fixed point $V \in \mathscr{C}\left(T_{K}\right)$, which is by construction a mild solution of 1.2 .

Besides, this solution is such that for all $T<T_{K},\left.V\right|_{(0, T)}$ is the fixed point of $\Lambda_{T}$ in $\mathscr{C}(T, K)$. Therefore, for $T$ such that $\left\|\mathbb{T}_{t} V_{0}\right\|_{\mathscr{C}(T)} \leqslant 1 /\left(8 C_{0}\right)$, it is easy to check from 8.6 that $\left\|\left.V\right|_{(0, T)}\right\|_{\mathscr{C}(T)} \leqslant$ $2\left\|\mathbb{T}_{t} V_{0}\right\|_{\mathscr{C}(T)}$, and thus goes to 0 as $T \rightarrow 0$. 
Furthermore, $V$ can be constructed as the limit of the sequence $V_{n+1}=\Lambda_{T_{K}} V_{n}$ for $n \in \mathbb{N}$, $V_{1}=0$, for which we have, for all $n \in \mathbb{N}, V_{n} \in \mathscr{C}\left(T_{K}, K\right)$. Elements of this sequence satisfies

$$
V_{n+1}(t)=\mathbb{T}_{t} V_{0}+\int_{0}^{t} \mathbb{T}_{t-s} \mathbb{P} \operatorname{div}\left(\mathbb{1}_{E}\left(\ell_{n}(s)-v_{n}(s)\right) \otimes v_{n}(s)\right) d s, \quad\left(t \in\left(0, T_{K}\right]\right)
$$

In particular, using (8.1), we get

$$
\begin{aligned}
\left\|V_{n+1}\right\|_{L^{\infty}\left(0, T_{K} ; \mathbb{X}^{3}\right)} & \leqslant C\left\|V_{0}\right\|_{\mathbb{X}^{3}}+C \sup _{t \in\left[0, T_{K}\right]}\left\{\int_{0}^{t} \frac{1}{(t-s)^{1 / 2} s^{1 / 2}} d s\right\}\left\|s^{1 / 4} V_{n}(s)\right\|_{L^{\infty}\left(0, T_{K} ; \mathbb{X}^{6}\right)}^{2} \\
& +C \sup _{t \in\left[0, T_{K}\right]}\left\{\int_{0}^{t} \frac{1}{(t-s)^{1 / 2} s^{1 / 2}} d s\right\}\left\|s^{1 / 2} V_{n}(s)\right\|_{L^{\infty}\left(0, T_{K} ; \mathbb{X}^{\infty}\right)}\left\|V_{n}\right\|_{L^{\infty}\left(0, T_{K} ; \mathbb{X}^{3}\right)} \\
& \leqslant C\left\|V_{0}\right\|_{\mathbb{X}^{3}}+C K^{2}+C K\left\|V_{n}\right\|_{L^{\infty}\left(0, T_{K} ; \mathbb{X}^{3}\right)} .
\end{aligned}
$$

Therefore, taking $K$ smaller if necessary to guarantee that $C K<1$, we get that the sequence $V_{n}$ is also uniformly bounded in $L^{\infty}\left(0, T_{K} ; \mathbb{X}^{3}\right)$, so that its limit $V$ is also bounded in $L^{\infty}\left(0, T_{K} ; \mathbb{X}^{3}\right)$. We then easily deduce by interpolation that, for all $p \in[3, \infty], t^{3 / 2(1 / 3-1 / p)} V \in C^{0}\left(\left[0, T_{K}\right] ; \mathbb{X}^{p}\right)$.

Besides, since $\left\|\mathbb{T}_{t} V_{0}\right\|_{\mathscr{C}(\infty)} \leqslant C_{1}\left\|V_{0}\right\|_{\mathbb{X}^{3}}$ according to estimates (7.2)-(7.3) for some constant $C_{1}$, if $\left\|V_{0}\right\|_{\mathbb{X}^{3}}$ is small enough (namely $\leqslant 1 /\left(8 C_{0} C_{1}\right)$ ), we can take $K=2 C\left\|V_{0}\right\|_{\mathbb{X}^{3}}$ and $T_{K}=\infty$. In this case, we have from the above computations that the above sequence $V_{n}$ stays in $\mathscr{C}(\infty, K)$ and stays bounded in $L^{\infty}\left(0, \infty ; \mathbb{X}^{3}\right)$ with $\left\|V_{n}\right\|_{L^{\infty}\left(0, \infty ; \mathbb{X}^{3}\right)} \leqslant C\left\|V_{0}\right\|_{\mathbb{X}^{3}}$. Consequently, when $\left\|V_{0}\right\|_{\mathbb{X}^{3}}$ is small enough, we can deduce by interpolation that there exists $C>0$ such that for all $n \in \mathbb{N}$,

$$
\sup _{p \in[3, \infty]}\left\|t^{3 / 2(1 / 3-1 / p)} V_{n}\right\|_{L^{\infty}\left(0, \infty ; \mathbb{X}^{p}\right)}+\left\|V_{n}\right\|_{\mathscr{C}(\infty)} \leqslant C\left\|V_{0}\right\|_{\mathbb{X}^{3}}
$$

and this also holds for the limit $V$ of the sequence $V_{n}$ :

$$
\sup _{p \in[3, \infty]}\left\|t^{3 / 2(1 / 3-1 / p)} V\right\|_{L^{\infty}\left(0, \infty ; \mathbb{X}^{p}\right)}+\|V\|_{\mathscr{C}(\infty)} \leqslant C\left\|V_{0}\right\|_{\mathbb{X}^{3}}
$$

Uniqueness. Let $V^{a}$ and $V^{b}$ be two mild solutions of $(1.2)$ with the same initial datum $V^{0}$ in the class $\mathscr{C}\left(T_{0}\right)$ such that for $\left\|V^{a}\right\|_{\mathscr{C}(T)}$ and $\left\|V^{b}\right\|_{\mathscr{C}(T)}$ go to 0 as $T \rightarrow 0$.

Then, setting

$$
e(t)=\left\|V^{a}-V^{b}\right\|_{\mathscr{C}(t)}, \quad t \in\left(0, T_{0}\right),
$$

according to 8.7), we have

$$
e(t) \leqslant C_{0}\left(\left\|V^{a}\right\|_{\mathscr{C}(t)}+\left\|V^{b}\right\|_{\mathscr{C}(t)}\right) e(t), \quad t \in\left(0, T_{0}\right) .
$$

Since $\left\|V^{a}\right\|_{\mathscr{C}(T)}$ and $\left\|V^{b}\right\|_{\mathscr{C}(T)}$ go to 0 as $T \rightarrow 0$, there exists $t_{0} \in\left(0, T_{0}\right]$, such that

$$
C_{0}\left(\left\|V^{a}\right\|_{\mathscr{C}\left(t_{0}\right)}+\left\|V^{b}\right\|_{\mathscr{C}\left(t_{0}\right)}\right)<1
$$

and thus $e\left(t_{0}\right)=0$, and $V^{a}$ and $V^{b}$ coincides on $\left[0, t_{0}\right]$. If $t_{0}<T_{0}$, it is easily seen that this argument can be repeated on time intervals of the form $\left[t_{*}, T\right]$ with $t^{*} \geqslant t_{0}$ : Using that for $t \in(0,1)$ such that $t+t_{*} \leqslant T_{0}$

$$
\left\|V^{a}\left(\cdot+t_{*}\right)\right\|_{\mathscr{C}(t)}+\left\|V^{b}\left(\cdot+t_{*}\right)\right\|_{\mathscr{C}(t)} \leqslant\left(\frac{t}{t+t_{*}}\right)^{1 / 4}\left(\left\|V^{a}\right\|_{\mathscr{C}\left(T_{0}\right)}+\left\|V^{b}\right\|_{\mathscr{C}\left(T_{0}\right)}\right) .
$$

we immediately have that there exists $t_{1}>0 \mathrm{such}$ that if $V^{a}$ and $V^{b}$ coincide in $\left[0, t_{*}\right]$ with $t_{*} \geqslant t_{0}$, then they coincide on $\left[0, \min \left\{t_{*}+t_{1}, T_{0}\right\}\right]$. This argument proves that $V^{a}$ and $V^{b}$ in fact coincide on the whole time interval $\left[0, T_{0}\right]$. 
The case of an initial datum in $\mathbb{X}^{q}$ for $q \in(1,3)$. Let $q \in(1,3)$ and $V_{0} \in \mathbb{X}^{q} \cap \mathbb{X}^{3}$ with $\left\|V_{0}\right\|_{\mathbb{X}^{3}} \leqslant \varepsilon_{0}$. Then we know that the solution $V$ of $\sqrt{1.2}$ is global in time and belong to $\mathscr{C}(\infty)$, and we know that the sequence given by $V_{1}=0$ and $V_{n+1}=\Lambda_{\infty}\left(V_{n}\right)$, i.e.

$$
\begin{aligned}
V_{n+1}(t) & =\mathbb{T}_{t} V_{0}+\int_{0}^{t} \mathbb{T}_{t-s} \mathbb{P} \operatorname{div}\left(\mathbb{1}_{E}\left(\ell_{n}(s)-v_{n}(s)\right) \otimes v_{n}(s)\right) d s, \quad(t \in(0, \infty]), \\
& =\mathbb{T}_{t} V_{0}+\int_{0}^{t} \mathbb{T}_{t-s} \mathbb{P}\left(\mathbb{1}_{E}\left(\ell_{n}(s)-v_{n}(s)\right) \cdot \nabla v_{n}(s)\right) d s, \quad(t \in(0, \infty]),
\end{aligned}
$$

converges to $V$ in $\mathscr{C}(\infty)$, and we have the estimates 8.16 .

To prove that $V$ is bounded in some class, it is enough to check that the sequence $\left(V_{n}\right)_{n \in \mathbb{N}}$ is uniformly bounded in this class.

Let us start by proving that $\min \left\{1, t^{1 / 2}\right\} \nabla v \in L^{\infty}\left(0, \infty ;\left[L^{q}(E)\right]^{9}\right)$. With $p \in(3, \infty)$ such that $1 / p+1 / q<1$

$$
\begin{aligned}
& \left\|\min \left\{1, t^{1 / 2}\right\} \nabla v_{n+1}\right\|_{L^{\infty}\left(0, \infty ;\left[L^{q}(E)\right]^{9}\right)} \leqslant C\left\|V_{0}\right\|_{\mathbb{X}^{q}} \\
& +C\left\|V_{0}\right\|_{\mathbb{X}^{3}} \sup _{t>0}\left\{\int_{0}^{t} \frac{\min \left\{1, t^{1 / 2}\right\} d s}{(t-s)^{3 /(2 p)+1 / 2} s^{3 / 2(1 / 3-1 / p)} \min \left\{1, s^{1 / 2}\right\}}\right\}\left\|\min \left\{1, t^{1 / 2}\right\} \nabla v_{n}\right\|_{L^{\infty}\left(0, \infty ;\left[L^{q}(E)\right]^{9}\right)} \\
& +C\left\|V_{0}\right\|_{\mathbb{X}^{3}} \sup _{t>0}\left\{\int_{0}^{t} \frac{\min \left\{1, t^{1 / 2}\right\} d s}{(t-s)^{1 / 2} \max \left\{1, s^{1 / 2}\right\} \min \left\{1, s^{1 / 2}\right\}}\right\}\left\|\min \left\{1, t^{1 / 2}\right\} \nabla v_{n}\right\|_{L^{\infty}\left(0, \infty ;\left[L^{q}(E)\right]^{9}\right)},
\end{aligned}
$$

where we used that, according to 8.16 ,

$$
\left\|t^{3 / 2(1 / 3-1 / p)} V_{n}\right\|_{L^{\infty}\left(0, \infty ; \mathbb{X}^{p}\right)}+\left\|\max \left\{1, t^{1 / 2}\right\} \ell_{n}\right\|_{L^{\infty}(0, \infty)} \leqslant C\left\|V_{0}\right\|_{\mathbb{X}^{3}} .
$$

We then use that

$$
\begin{aligned}
& \sup _{t>0}\left\{\int_{0}^{t} \frac{\min \left\{1, t^{1 / 2}\right\}}{(t-s)^{3 /(2 p)+1 / 2} s^{3 / 2(1 / 3-1 / p)} \min \left\{1, s^{1 / 2}\right\}} d s\right\} \leqslant C, \\
& \sup _{t>0}\left\{\int_{0}^{t} \frac{\min \left\{1, t^{1 / 2}\right\}}{(t-s)^{1 / 2} \max \left\{1, s^{1 / 2}\right\} \min \left\{1, s^{1 / 2}\right\}} d s\right\} \leqslant C,
\end{aligned}
$$

which can be proved along the same lines as in 8.10-8.11 -8.12 - 8.13.

This allows to deduce

$$
\begin{aligned}
& \left\|\min \left\{1, t^{1 / 2}\right\} \nabla v_{n+1}\right\|_{L^{\infty}\left(0, \infty ;\left[L^{q}(E)\right]^{9}\right)} \\
& \quad \leqslant C\left\|V_{0}\right\|_{\mathbb{X}^{q}}+C\left\|V_{0}\right\|_{\mathbb{X}^{3}}\left\|\min \left\{1, t^{1 / 2}\right\} \nabla v_{n}\right\|_{L^{\infty}\left(0, \infty ;\left[L^{q}(E)\right]^{9}\right)} .
\end{aligned}
$$

Accordingly, if $\left\|V_{0}\right\|_{\mathbb{X}^{3}}$ is small enough, we have that

$$
\left\|\min \left\{1, t^{1 / 2}\right\} \nabla v_{n+1}\right\|_{L^{\infty}\left(0, \infty ;\left[L^{q}(E)\right]^{9}\right)} \leqslant C\left\|V_{0}\right\|_{\mathbb{X}^{q}}+\frac{1}{2}\left\|\min \left\{1, t^{1 / 2}\right\} \nabla v_{n}\right\|_{L^{\infty}\left(0, \infty ;\left[L^{q}(E)\right]^{9}\right)},
$$

so that the sequence $\left(\min \left\{1, t^{1 / 2}\right\} \nabla v_{n}\right)_{n \in \mathbb{N}}$ is uniformly bounded in $L^{\infty}\left(0, \infty ;\left[L^{q}(E)\right]^{9}\right)$, and passing to the limit $n \rightarrow \infty, \min \left\{1, t^{1 / 2}\right\} \nabla v$ belongs to $L^{\infty}\left(0, \infty ;\left[L^{q}(E)\right]^{9}\right)$.

Accordingly, $\min \left\{t^{1 / 2}, t\right\}\left(v_{n}-\ell_{n}\right) \cdot \nabla v_{n}$ belongs to $L^{\infty}\left(0, \infty ; L^{q}\left(\mathbb{R}^{3}\right)\right)$ and we can use Lemma 8.1 for $q_{0} \geqslant \max \{q, 3 / 2\}$.

Then we set $q_{0}=\max \{q, 3 / 2\}$, and we next prove that $t^{3 / 2\left(1 / q-1 / q_{0}\right)} V \in L^{\infty}\left(0, \infty ; \mathbb{X}^{q_{0}}\right)$ and $t^{3 / 2(1 / q-1 / 6)} V \in L^{\infty}\left(0, \infty ; \mathbb{X}^{6}\right)$. In order to do that, again we look at the sequence $\left(t^{3 / 2\left(1 / q-1 / q_{0}\right)} V_{n}\right)_{n \in \mathbb{N}}$ in $L^{\infty}\left(0, \infty ; \mathbb{X}^{q_{0}}\right)$ :

$$
\begin{aligned}
& \left\|t^{3 / 2\left(1 / q-1 / q_{0}\right)} V_{n+1}\right\|_{L^{\infty}\left(0, \infty ; \mathbb{X}^{q_{0}}\right)} \leqslant C\left\|V_{0}\right\|_{\mathbb{X}^{q}} \\
& +C \sup _{t>0}\left\{\int_{0}^{t} \frac{t^{3 / 2\left(1 / q-1 / q_{0}\right)}}{(t-s)^{1 / 2} s^{1 / 2} s^{3 / 2\left(1 / q-1 / q_{0}\right)}} d s\right\}\left\|V_{n}\right\|_{\mathscr{C}(\infty)}\left\|t^{3 / 2\left(1 / q-1 / q_{0}\right)} V_{n}\right\|_{L^{\infty}\left(0, \infty ; \mathbb{X}^{q_{0}}\right)} \\
& \leqslant C\left\|V_{0}\right\|_{\mathbb{X}^{q}}+C\left\|V_{0}\right\|_{\mathbb{X}^{3}}\left\|t^{3 / 2\left(1 / q-1 / q_{0}\right)} V_{n}\right\|_{L^{\infty}\left(0, \infty ; \mathbb{X}^{q_{0}}\right)} .
\end{aligned}
$$


This implies that for $\left\|V_{0}\right\|_{\mathbb{X}^{3}}$ small enough, the sequence $\left(t^{3 / 2\left(1 / q-1 / q_{0}\right)} V_{n}\right)_{n \in \mathbb{N}}$ is uniformly bounded in $L^{\infty}\left(0, \infty ; \mathbb{X}^{q_{0}}\right)$ by $C\left\|V_{0}\right\|_{\mathbb{X}^{q}}$.

Similarly, we consider the norm of $t^{3 / 2(1 / q-1 / 6)} V_{n}$ in $L^{\infty}\left(0, \infty ; \mathbb{X}^{6}\right)$ :

$$
\begin{aligned}
& \left\|t^{3 / 2(1 / q-1 / 6)} V_{n+1}\right\|_{L^{\infty}\left(0, \infty ; \mathbb{X}^{6}\right)} \leqslant C\left\|V_{0}\right\|_{\mathbb{X}^{q}} \\
& +C \sup _{t>0}\left\{\int_{0}^{t / 2} \frac{t^{3 / 2(1 / q-1 / 6)}}{(t-s)^{1 / 2+3 / 2\left(1 / q_{0}-1 / 6\right)} s^{1 / 2} s^{3 / 2\left(1 / q-1 / q_{0}\right)}} d s\right\}\left\|V_{n}\right\|_{\mathscr{C}(\infty)}\left\|t^{3 / 2\left(1 / q-1 / q_{0}\right)} V_{n}\right\|_{L^{\infty}\left(0, \infty ; \mathbb{X}^{q_{0}}\right)} \\
& +C \sup _{t>0}\left\{\int_{t / 2}^{t} \frac{t^{3 / 2(1 / q-1 / 6)}}{(t-s)^{1 / 2} s^{1 / 2} s^{3 / 2(1 / q-1 / 6)}} d s\right\}\left\|V_{n}\right\|_{\mathscr{C}(\infty)}\left\|t^{3 / 2(1 / q-1 / 6)} V_{n}\right\|_{L^{\infty}\left(0, \infty ; \mathbb{X}^{6}\right)} \\
& \leqslant C\left\|V_{0}\right\|_{\mathbb{X}^{q}}+C\left\|V_{0}\right\|_{\mathbb{X}^{3}}\left\|V_{0}\right\|_{\mathbb{X}^{q}}+C\left\|V_{0}\right\|_{\mathbb{X}^{3}}\left\|t^{3 / 2(1 / q-1 / 3)} V_{n}\right\|_{L^{\infty}\left(0, \infty ; \mathbb{X}^{6}\right)} .
\end{aligned}
$$

Accordingly, if $\left\|V_{0}\right\|_{\mathbb{X}^{3}}$ is small enough, the sequence $\left(t^{3 / 2(1 / q-1 / 6)} V_{n}\right)_{n \in \mathbb{N}}$ is bounded in $L^{\infty}\left(0, \infty ; \mathbb{X}^{6}\right)$ by $C\left\|V_{0}\right\|_{\mathbb{X}^{q}}+C\left\|V_{0}\right\|_{\mathbb{X}^{3}}\left\|V_{0}\right\|_{\mathbb{X}^{q}}$.

Therefore, the limit $V$ of the sequence $\left(V_{n}\right)_{n \in \mathbb{N}}$ satisfies $t^{3 / 2\left(1 / q-1 / q_{0}\right)} V \in L^{\infty}\left(0, \infty ; \mathbb{X}^{q_{0}}\right)$ and $t^{3 / 2(1 / q-1 / 6)} V \in L^{\infty}\left(0, \infty ; \mathbb{X}^{6}\right)$.

Then, we use that $V$ satisfies

$$
V(t)=\mathbb{T}_{t} V_{0}+\int_{0}^{t} \mathbb{T}_{t-s} \mathbb{P} \operatorname{div}\left(\mathbb{1}_{E}(\ell(s)-v(s)) \otimes v(s)\right) d s, \quad(t \in(0, \infty]),
$$

and the fact that, from our previous computations, there exists $C>0$ such that for all $s>0$,

$$
\begin{aligned}
& \|(\ell(s)-v(s)) \otimes v(s)\|_{6} \leqslant 2\|V(s)\|_{\infty}\|V(s)\|_{6} \leqslant \frac{C}{s^{1 / 2} s^{3 / 2(1 / q-1 / 6)}} \leqslant \frac{C}{s^{3 /(2 q)+1 / 4}}, \\
& \|(\ell(s)-v(s)) \otimes v(s)\|_{q_{0}} \leqslant 2\|V(s)\|_{\infty}\|V(s)\|_{q_{0}} \leqslant \frac{C}{s^{1 / 2} s^{3 / 2\left(1 / q-1 / q_{0}\right)}} \leqslant \frac{C}{s^{1 / 2+3 / 2\left(1 / q-1 / q_{0}\right)}} .
\end{aligned}
$$

Next, using Lemma 8.1 with $q_{0}$ and $p_{0}=\infty$ for $s \in(0, t / 2)$, and with 6 and $p_{0}=\infty$ for $s \in(t / 2, t)$, we have

$$
\begin{aligned}
& \left\|t^{3 /(2 q)} V(t)\right\|_{L^{\infty}\left(0, \infty ; \mathbb{X}^{\infty}\right)} \leqslant C\left\|V_{0}\right\|_{\mathbb{X}^{q}} \\
& +C \sup _{t>0}\left\{\int_{0}^{t / 2} \frac{t^{3 /(2 q)}}{(t-s)^{1 / 2+3 /\left(2 q_{0}\right)} s^{1 / 2+3 / 2\left(1 / q-1 / q_{0}\right)}} d s+\int_{t / 2}^{t} \frac{t^{3 /(2 q)}}{(t-s)^{3 / 4} s^{3 / 2 q+1 / 4}} d s\right\} \\
& \leqslant C<\infty .
\end{aligned}
$$

Therefore, $t^{3 /(2 q)} V(t) \in L^{\infty}\left(0, \infty ; \mathbb{X}^{\infty}\right)$. Since we have already proved that $t^{3 / 2\left(1 / q-1 / q_{0}\right)} V \in$ $L^{\infty}\left(0, \infty ; \mathbb{X}^{q_{0}}\right)$, we conclude by interpolation that for all $p \in\left[q_{0}, \infty\right], t^{3 / 2(1 / q-1 / p)} V \in L^{\infty}\left(0, \infty ; \mathbb{X}^{p}\right)$.

\section{Concluding remarks and open questions}

The main result in this paper, namely Theorem 1.1, concerns the wellposedness of the system modelling the motion of a rigid ball in a viscous incompressible fluid filling the remaining part of $\mathbb{R}^{3}$ and asserts that the position of the centre of the ball tends, when $t \rightarrow \infty$, to some position $h_{\infty} \in \mathbb{R}^{3}$. This result differs from those previously obtained in two space dimensions in [6], or for a simplified $1 D$ model in [26], where it has been shown that the distance of the centre of the ball to the origin tends to $+\infty$ when $t \rightarrow \infty$. Several open questions seem natural in view of our results.

One of the most challenging ones, for which we have no track at this stage, is determining $h_{\infty}$ from the initial data. 
Another natural question is the generalization of Theorem 1.1 for a body of arbitrary shape. When the rigid body is not a ball, writing the equations in a fixed domain requires the use of more delicate changes of variables, since it has to include the rotation of the body. There are basically two ways of doing that: one consists in setting $v(t, x)=Q^{*}(t) u(t, h(t)+Q(t) x)$, where $Q(t)$ is the rotation matrix of the body, that is the solution of $\dot{Q}(t) Q^{*}(t) x=\omega(t) \times x$ starting from $Q(0)=I d$. The problem is that such change of frame would induce in the fixed frame a term of the form $(\omega \times x) \cdot \nabla v$ which our estimate does not allow to handle since the identity mapping does not belong to $L^{\infty}(E)$. The alternative approach proposed in [4, which consists in constructing a change of variable which follows the structure in a neighbourhood of it and equals the identity far from the body, seems more suitable to deal with the non-linear terms. However, this change of variable introduces a lot of delicate terms which we do not know how to handle in the above setting so far. In fact, even in two space dimensions, the existing results (see 6]) provide an analysis of the motion of a rigid body in a viscous incompressible fluid in $\mathbb{R}^{2}$ only in the case when the rigid body is a disk.

Finally, let us mention that the counterparts in two space dimensions of some of our results in Sections 47 have been used in Takahashi and Lacave [16] to study the behaviour of solutions of (1.2) when the radius of the rigid ball tends to zero (see also He and Iftimie [10] and references therein). We believe that the approach in [16] can be adapted to the three dimensional case by using our results on the fluid structure-semigroup and its generator, but this deserves further work.

Acknowledgements. The authors are indebted to Toshiaki Hishida for his suggestions, which had led to many improvements of our work.

\section{A Proof of Proposition 3.2}

We first show that every $u \in\left[L^{q}(\Omega)\right]^{3}$ can be written in the form $u=v+w_{1}+w_{2}$, with $v \in \mathbb{X}^{q}(\Omega)$, $w_{1} \in G_{1}^{q}(\Omega)$ and $w_{2} \in G_{2}^{q}(\Omega)$. To this aim, let $q_{1}$ be the solution of the problem

$$
\Delta q_{1}=\operatorname{div} u \text { in } \Omega, \quad q_{1}=0 \text { on } \partial \Omega .
$$

Thus $q_{1} \in W_{0}^{1, q}(\Omega)$ and setting $w_{1}=\nabla q_{1}$, we clearly have $w_{1} \in G_{1}^{q}(\Omega)$. Since we are looking for $v \in \mathbb{X}^{q}(\Omega)$, we know that

$$
v=\ell_{v}+\omega_{v} \times x \text { for } x \in \mathcal{O},
$$

for some $\ell_{v} \in \mathbb{R}^{3}$ and $\omega_{v} \in \mathbb{R}^{3}$. We set

$$
\varphi(x)=u(x)-\nabla q_{1}(x)-\ell_{v}-\omega_{v} \times x \quad x \in \mathcal{O},
$$

and

$$
w_{2}= \begin{cases}\nabla q_{2} & \text { in } E_{\Omega} \\ \varphi & \text { in } \mathcal{O}\end{cases}
$$

Since we are looking for $w_{2} \in G_{2}^{q}(\Omega)$ we require

$$
\ell_{v}=\frac{1}{m} \int_{\mathcal{O}}\left(u-\nabla q_{1}-\varphi\right) \mathrm{d} x=\frac{1}{m}\left[\int_{\mathcal{O}}\left(u-\nabla q_{1}\right) \mathrm{d} x+\int_{\partial \mathcal{O}} q_{2} \nu \mathrm{d} \gamma\right]
$$

and

$$
\omega_{v}=-\frac{1}{\mathcal{J}} \int_{\mathcal{O}}\left(u-\nabla q_{1}-\varphi\right) \times x \mathrm{~d} x=-\frac{1}{\mathcal{J}}\left[\int_{\mathcal{O}}\left(u-\nabla q_{1}\right) \times x \mathrm{~d} x+\int_{\partial \mathcal{O}} q_{2} \nu \times x \mathrm{~d} \gamma\right],
$$

where $m$ and $\mathcal{J}$ are defined in 3.9 . 
Now we define $q_{2}$ as the solution of the Neumann problem

$$
\begin{cases}\Delta q_{2}=0 & \text { in } E_{\Omega}, \\ \frac{\partial q_{2}}{\partial n}=\left(u-\nabla q_{1}\right) \cdot n & \text { on } \partial \Omega, \\ \frac{\partial q_{2}}{\partial n}=\left(u-\nabla q_{1}\right) \cdot n-\left(\ell_{v}+\omega_{v} \times x\right) \cdot n & \text { on } \partial \mathcal{O},\end{cases}
$$

where $\ell_{v}$ and $\omega_{v}$ are defined in A.4 and A.5, respectively. Note that, $q_{2}$ solves a Laplace equation with non-local boundary condition. As shown below, we have that $q_{2} \in W^{1, q}\left(E_{\Omega}\right)$ and there exists a constant $C$ depending on $q, \Omega$ and $\mathcal{O}$ such that

$$
\left\|q_{2}\right\|_{1, q, E_{\Omega}} \leqslant C\|u\|_{q, \Omega} .
$$

In this case we can determine $\ell_{v}$ and $\omega_{v}$ from A.4 and A.5 respectively. Consequently, we obtain $\varphi$ and $w_{2}$ from $\mathrm{A} .2$ and $\mathrm{A} .3$ respectively. In particular, we have that $w_{2} \in G_{2}^{q}(\Omega)$ and by setting $v=u-w_{1}-w_{2}$ we can verify that $v \in \mathbb{X}^{q}(\Omega)$.

We still have to prove that $q_{2} \in W^{1, q}(\Omega)$ and A.7 holds. If $q=2$, this is a consequence of Lax-Milgram Theorem (see for instance [5, Lemma 1]). If $q \neq 2$, we employ a density argument. Assume that $u \in\left[C_{0}^{\infty}(\Omega)\right]^{3}$ and $q_{2}$ solves A.6. Then there exists a constant $C$ depending only on $q, \Omega$ and $\mathcal{O}$ such that

$$
\left\|q_{2}\right\|_{1, q, E_{\Omega}} \leqslant C\left(\|u\|_{q, \Omega}+\left|\ell_{v}\right|+\left|\omega_{v}\right|\right) .
$$

Next, by following the arguments of the proof of Theorem 2.2 in [27], we have

$$
\left|\ell_{v}\right|+\left|\omega_{v}\right| \leqslant C\|u\|_{q, \Omega},
$$

where $C$ is a positive constant depending only on $q, \Omega$ and $\mathcal{O}$. The above two estimates yield that there exists a positive constant $C$, depending only on $q, \Omega$ and $\mathcal{O}$, such that estimate A.7 holds. Thus the conclusion follows by a density argument. This completes the proof of the existence of a decomposition with the required properties. The proof of uniqueness of the decomposition is similar to that of [27, Theorem 2.2].

\section{References}

[1] M. E. BogovskiĬ, Solution of the first boundary value problem for an equation of continuity of an incompressible medium, Dokl. Akad. Nauk SSSR, 248 (1979), pp. 1037-1040.

[2] W. Borchers AND H. SoHr, On the semigroup of the Stokes operator for exterior domains in $L^{q}$-spaces, Math. Z., 196 (1987), pp. 415-425.

[3] F. Crispo and P. Maremonti, An interpolation inequality in exterior domains, Rend. Sem. Mat. Univ. Padova, 112 (2004), pp. 11-39.

[4] P. Cumsille And T. TAKahashi, Wellposedness for the system modelling the motion of a rigid body of arbitrary form in an incompressible viscous fluid, Czechoslovak Math. J., 58(133) (2008), pp. 961-992.

[5] M. Dashti And J. C. Robinson, The motion of a fluid-rigid disc system at the zero limit of the rigid disc radius, Arch. Ration. Mech. Anal., 200 (2011), pp. 285-312.

[6] S. Ervedoza, M. Hillairet, And C. LACAVe, Long-time behavior for the two-dimensional motion of a disk in a viscous fluid, Comm. Math. Phys., 329 (2014), pp. 325-382.

[7] M. Geissert, K. GöTze, And M. Hieber, $L^{p}$-theory for strong solutions to fluid-rigid body interaction in Newtonian and generalized Newtonian fluids, Trans. Amer. Math. Soc., 365 (2013), pp. 1393-1439. 
[8] Y. Giga And H. Sohr, On the Stokes operator in exterior domains, J. Fac. Sci. Univ. Tokyo Sect. IA Math., 36 (1989), pp. 103-130.

[9] J. Happel AND H. BrenneR, Low Reynolds number hydrodynamics with special applications to particulate media, Prentice-Hall, Inc., Englewood Cliffs, N.J., 1965.

[10] J. He And D. Iftimie, On the small rigid body limit in 3d incompressible flows, arXiv preprint arXiv:1812.09196, (2018).

[11] T. HishidA, Large time behavior of a generalized Oseen evolution operator, with applications to the Navier-Stokes flow past a rotating obstacle, Mathematische Annalen, 372 (2018), pp. 915949 .

$[12] —$ Decay estimates of gradient of a generalized Oseen evolution operator arising from timedependent rigid motions in exterior domains, arXiv preprint arXiv:1908.04080, (2019).

[13] H. IwASHITA, $L_{q}-L_{r}$ estimates for the solutions of the nonstationary Stokes equations in an exterior domain and the Navier-Stokes initial value problems in $L_{q}$ spaces, Math. Ann., 285 (1989), pp. 265-288.

[14] T. KATO, Strong $L^{p}$-solutions of the Navier-Stokes equation in $\mathbf{R}^{m}$, with applications to weak solutions, Math. Z., 187 (1984), pp. 471-480.

[15] K. KoIKE, Long-time behavior of a point mass in a one-dimensional viscous compressible fluid and pointwise estimates of solutions, J. Differential Equations, 271 (2021), pp. 356-413.

[16] C. Lacave And T. TAKahashi, Small moving rigid body into a viscous incompressible fluid, Archive for Rational Mechanics and Analysis, 223 (2017), pp. 1307-1335.

[17] A. LunARdi, Interpolation theory, Appunti. Scuola Normale Superiore di Pisa (Nuova Serie). [Lecture Notes. Scuola Normale Superiore di Pisa (New Series)], Edizioni della Normale, Pisa, second ed., 2009.

[18] D. Maity AND M. TUCSNAK, $L^{p}-L^{q}$ maximal regularity for some operators associated with linearized incompressible fluid-rigid body problems, in Mathematical analysis in fluid mechanicsselected recent results, vol. 710 of Contemp. Math., Amer. Math. Soc., Providence, RI, 2018, pp. $175-201$.

[19] P. Maremonti and V. A. Solonnikov, On nonstationary Stokes problem in exterior domains, Ann. Scuola Norm. Sup. Pisa Cl. Sci. (4), 24 (1997), pp. 395-449.

[20] M. McCracken, The resolvent problem for the Stokes equations on halfspace in $L^{p}$, SIAM Journal on Mathematical Analysis, 12 (1981), pp. 201-228.

[21] D. Serre, Chute libre d'un solide dans un fluide visqueux incompressible. Existence, Japan J. Appl. Math., 4 (1987), pp. 99-110.

[22] T. TAKahashi, Analysis of strong solutions for the equations modeling the motion of a rigidfluid system in a bounded domain, Adv. Differential Equations, 8 (2003), pp. 1499-1532.

[23] T. Takahashi and M. Tucsnak, Global strong solutions for the two dimensional motion of an infinite cylinder in a viscous fluid, J. Math. Fluid Mech., 6 (2004), pp. 53-77.

[24] R. Temam, Problèmes mathématiques en plasticité, vol. 12 of Méthodes Mathématiques de l'Informatique [Mathematical Methods of Information Science], Gauthier-Villars, Montrouge, 1983.

[25] H. Triebel, Interpolation theory, Function Spaces, Differential Operators, 2nd edition, Johann Ambrosius Barth, Heidelberg, (1995).

[26] J. L. VÁzquez AND E. ZuAzuA, Large time behavior for a simplified 1D model of fluid-solid interaction, Comm. Partial Differential Equations, 28 (2003), pp. 1705-1738.

[27] Y. WANG AND Z. XIN, Analyticity of the semigroup associated with the fluid-rigid body problem and local existence of strong solutions, Journal of Functional Analysis, 261 (2011), pp. 25872616. 